\title{
X-ray Diagnostics of Accretion Plasmas in Selected Soft Polars
}

\author{
Dissertation \\ zur Erlangung des Doktorgrades \\ der Mathematisch-Naturwissenschaften Fakultäten \\ der Georg-August-Universität zu Göttingen
}

\author{
vorgelegt von \\ Iris Traulsen \\ aus Stuttgart
}

Göttingen, Februar 2009 
D7

Referent:

Prof. Dr. S. Dreizler

Korreferent:

Prof. Dr. W. Kollatschny

Tag der mündlichen Prüfung:

6. März 2009 


\section{ZUSAMMENFASSUNG}

\section{Akkretionsplasmen in Polaren mit ausgeprägter weicher Röntgenstrahlung}

Akkretionsprozesse spielen während vielerlei Entwicklungsphasen von Sternen und in vielerlei Größenordnungen eine Rolle. Sie treten beispielsweise während der Sternentstehung auf, in Aktiven Galaxien oder in engen Doppelsternsystemen, deren eine Komponente ins Endstadium seiner Entwicklung eingetreten ist, den so genannten Röntgendoppelsternen und Kataklysmischen Veränderlichen. Magnetische kataklysmische Veränderliche vom Typ der AMHer-Sterne (Polare) bestehen aus einem Weißen Zwerg mit einem starken Magnetfeld und einem Begleiter geringer Masse, der sein Roche-Volumen ausfüllt. Der Weiße Zwerg akkretiert Materie vom Sekundärstern, die entlang der Magnetfeldlinien auf seine magnetischen Pole geleitet wird. Oberhalb des Weißen Zwergs bildet sich eine Stoßfront aus, und die Materie heizt sich auf hohe Temperaturen auf. Im Bereich zwischen Stoßfront und Photosphäre wird das Plasma in der Akkretionssäule abgebremst und abgekühlt, in erster Linie durch Emission von optischer Zyklotron- und harter Röntgen-Bremsstrahlung. Weicher Röntgen- und ultravioletter Fluss entstehen in der geheizten Photosphäre, in der die Strahlung der Akkretionssäule reprozessiert wird. Weiche und harte Röntgenemission sollten sich folglich in etwa die Waage halten. Einige Systeme weisen jedoch eine deutlich höhere Leuchtkraft im weichen Röntgenbereich auf, die mit einer besonders effektiven Zyklotronkühlung und mit inhomogener Akkretion zusammenhängen dürfte.

Zwei magnetische kataklysmische Variable mit ausgeprägtem weichem Röntgenfluss stehen im Mittelpunkt der vorliegenden Arbeit. Für beide Systeme, AI Tri und QS Tel, wurden Röntgendaten mit dem Weltraumteleskop XMM-Newton und optische Daten mit verschiedenen bodengebundenen Teleskopen aufgenommen, die die Grundlage für eine eingehende photometrische und spektroskopische Analyse bilden. Die üblicherweise gebräuchlichen Spektralmodelle, bestehend aus einer Schwarzkörper- und einer Plasmakomponen- 
te, wurden erweitert, um einen großen Bereich von Temperaturen abzudecken und somit dem komplexen Aufbau der Emissionsregionen Rechnung zu tragen. Ein deutlicher Überschuss an weicher Röntgenstrahlung kennzeichnet die beobachteten Objekte. Ihre Lichtkurven besitzen eine stark variable Struktur, Zeichen für die zeitlich und räumlich inhomogenen Akkretionsvorgänge. Die Beobachtungsdaten des langperiodischen Polaren AI Tri sind durch die Strahlung aus einer einzelnen Akkretionsregion geprägt, die für ein Zehntel der Orbitalperiode durch den Akkretionsstrom bedeckt wird. Die Spektren lassen sich durch Schwarzkörperkomponenten mit Temperaturen von bis zu $44.0_{-3.2}^{+3.3} \mathrm{eV}$ und Emission aus einem Plasma mit solarer Elementhäufigkeit bei Temperaturen zwischen $0.8_{-0.2}^{+0.4} \mathrm{keV}$ und $20.3_{-5.1}^{+8.7} \mathrm{keV}$ wiedergeben. Während zweier Beobachtungen zeigte AI Tri ein 'irreguläres' Verhalten: Die Röntgendaten wurden noch wesentlich stärker von weicher Röntgenstrahlung dominiert, die optischen Lichtkurven waren um $\Delta \phi \simeq 0.2$ phasenverschoben. Der zweite Polar, QS Tel, wechselt zwischen Phasen von Akkretion auf einen einzelnen und auf beide Pole. Während der Beobachtung mit XMM-Newton wies QS Tel kaum Fluss bei Energien oberhalb von $2 \mathrm{keV}$ auf, und in erster Linie war die weiche Röntgenstrahlung produzierende Akkretionsregion vorherrschend. Die zweite Region mit Flussmaximum im optischen und ultravioletten Bereich zeigte nur geringe Aktivität. Zahlreiche Metalllinien lassen sich in den Röntgenspektren identifizieren, die am besten bei niedrigen Temperaturen unter $5 \mathrm{keV}$ und um $19.5_{-4.2}^{+3.7} \mathrm{eV}$ beschrieben werden. Im Vergleich zwischen verschiedenen spektralen Fits wird bei beiden Objekten offensichtlich, dass geeignete Modelle ein hinreichend großes Dichte- und Temperaturintervall überdecken müssen. Dabei beeinflusst die Wahl des Modells deutlich das quantitative Verhältnis zwischen weicher und harter Röntgenstrahlung, das sich daraus ableiten lässt. 


\section{ABSTRACT}

Accretion processes occur during diverse stages of stellar evolution and on diverse scales, as for example in star formation, in active galactic nuclei, or in close binaries with a compact late-type component; the X-ray binaries and cataclysmic variables. Magnetic cataclysmic variables of AM Her type, also called polars, comprise a white dwarf with a strong magnetic field, accreting matter from a Roche-lobe filling low-mass secondary. The accretion stream is channeled along the field lines towards the magnetic poles of the white dwarf. Forming a strong shock at high temperatures above the star surface, it is decelerated and cooled by optical cyclotron radiation and by bremsstrahlung in the hard X-ray regime. Soft X-ray and ultraviolet radiation arise from the heated photosphere, where the hard emission is reprocessed. Although the two X-ray flux components would be expected to balance each other according to the standard picture, several systems show a distinct dominance of soft over hard $\mathrm{X}$-radiation. In these polars, cyclotron emission is believed to be the main cooling process, associated with inhomogeneous and time-variable accretion.

The work concentrates on the two soft magnetic cataclysmic variables AI Tri and QS Tel. The systems have been observed during high and intermediate high states of accretion with the X-ray satellite XMM-Newton and with optical telescopes; basis for a detailed photometric and spectroscopic analysis. In order to account for the complex structure of the X-ray emitting regions, the usual black body and plasma models have been expanded to multi-temperature approaches in the spectral fits. A high soft-to-hard X-ray flux ratio characterizes both objects. Highly variable on short time scales, their light curves reflect the inhomogeneous, 'blobby' column accretion. Emission from one main accretion region shapes the observational data of the long-period polar AI Tri. The region is eclipsed by the accretion stream for a tenth of the orbital cycle. The best spectral fit yields temperatures of up to $44.0_{-3.2}^{+3.3} \mathrm{eV}$ for the soft component and between $0.8_{-0.2}^{+0.4} \mathrm{keV}$ and $20.3_{-5.1}^{+8.7} \mathrm{keV}$ for the plasma component with nearly solar element abundances. During two observations, AI Tri was found in an irregular mode of accretion with a considerably softer X-ray 
spectrum and a phase shift of the optical light curves by $\Delta \phi \simeq 0.2$. From the second analyzed polar, QS Tel, hardly any counts above energies of $2 \mathrm{keV}$ are detected. Known to switch between one-pole and two-pole accretion, it was found in a stage dominated by emission from the X-ray bright pole during the XMM-Newton observation. The second pole, mainly seen at optical and ultraviolet wavelengths, showed less activity. Rich metal lines turn up in the X-ray spectra of QS Tel, which are composed of cool plasma and black body components at temperatures below $5 \mathrm{keV}$ and around $19.5_{-4.2}^{+3.7} \mathrm{eV}$ respectively. The comparison of different fits to the spectra of the two objects demonstrates the need for models considering the wide range of densities and temperatures in the accretion plasmas. The impact of the choice of the spectral models on the soft-to-hard ratios in polars is discussed. 


\section{CONTENTS}

List of Objects $\quad$ xi

List of Figures xiii

List of Tables $\quad$ XV

1 Introduction 1

2 Magnetic cataclysmic variables in X-rays 4

2.1 Close binary systems . . . . . . . . . . . . 5

2.2 Evolution of compact binaries . . . . . . . . . . 6

2.3 The configuration of cataclysmic variables . . . . . . . . 8

2.4 The influence of the magnetic field: Polars . . . . . . . . . . 12

2.5 X-ray diagnostics of MCVs . . . . . . . . . . . . . . 15

2.5.1 X-ray emission from polars . . . . . . . . . . 16

2.5.2 Hydrodynamic models . . . . . . . . . . . . . . . 17

2.5.3 Soft X-ray dominated systems . . . . . . . . . . . . 19

3 Modeling the X-ray spectra of MCVs 21

3.1 Spectral fitting by means of XSPEC . . . . . . . . . . 21

3.1 .1 Black body radiation . . . . . . . . . . . . . . 23

3.1.2 Thermal plasma emission . . . . . . . . . . . 24

3.1 .3 Cooling flow models . . . . . . . . . . . . . 25

3.1.4 Galactic and intrinsic absorption . . . . . . . . . 26

3.2 A multi-temperature black body approach to the accretion region 27

3.3 A multi-temperature plasma approach to the accretion column 29

3.4 Caveats and conclusions . . . . . . . . . . . . . . 31 
4 X-ray instrumentation $\quad 33$

4.1 Missions overview . . . . . . . . . . . . . . . . . . 33

4.1 .1 XMM-Newton . . . . . . . . . . . . . 35

4.1 .2 ROSAT . . . . . . . . . . . . . . 42

4.2 Probing the XMM-Newton calibration at low energies . . . . . 43

4.2 .1 Objects . . . . . . . . . . . . . . . . 44

4.2.2 Data selection and method . . . . . . . . . . . 46

4.2 .3 Results . . . . . . . . . . . . . . . . . . . . . . . . 48

4.2 .4 Conclusions . . . . . . . . . . . . . 51

5 The long-period polar AI Tri 52

5.1 Observations and data reduction $\ldots \ldots \ldots \ldots$

5.1 .1 XMM-Newton . . . . . . . . . . . . . . 53

5.1 .2 Dealing with the flaring background . . . . . . 55

5.1 .3 ROSAT HRI . . . . . . . . . . . . . . . . . . 58

5.1 .4 Optical observations . . . . . . . . . . . . . 58

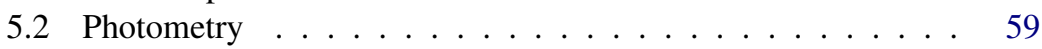

5.2 .1 XMM-Newton X-ray $\ldots \ldots \ldots$

5.2 .2 XMM-Newton UV . . . . . . . . . . . . . . . . . . 59

5.2 .3 Optical . . . . . . . . . . . . . 62

5.2.4 Irregular mode on August 17, $2005 \ldots \ldots 5$

5.3 X-ray spectroscopy . . . . . . . . . . . . . . . . 67

5.3.1 Phase-resolved modeling . . . . . . . . . . . 71

5.3.2 XMM-Newton data on August 15, $2005 \ldots . . . . .72$

5.4 Discussion . . . . . . . . . . . . . . . . 73

5.4.1 The flaring structure of the light curves . . . . . . . 73

5.4.2 The nature of the soft X-ray minima . . . . . . 76

5.4 .3 Geometry of the system . . . . . . . . . . . 82

5.4 .4 On the soft-to-hard ratio . . . . . . . . . . . . . 83

5.5 Conclusions . . . . . . . . . . . . . . 85

6 QS Tel - A polar changing state $\quad 86$

6.1 XMM-Newton and optical observations . . . . . . . . 87

6.2 Radial velocity variations . . . . . . . . . . . . . . . . . . . . . 88

6.3 Multi-wave band photometry . . . . . . . . . . . . . 90

6.4 The XMM-Newton/EPIC spectra . . . . . . . . . . . . . . . . 94

6.4.1 The orbital mean spectrum . . . . . . . . . . . . . 94

6.4 .2 X-ray bright phases . . . . . . . . . . . 97 
6.4 .3 X-ray faint phases . . . . . . . . . . . . . . . . . . . . . . 98

6.5 Discussion and conclusions . . . . . . . . . . . . . . 99

6.5.1 The soft X-ray excess _. . . . . . . . . . . . . . . 99

6.5.2 The X-ray light curve dips . . . . . . . . . . . . . . . . 100

6.5 .3 Accretion state . . . . . . . . . . . . . 101

$\begin{array}{lll}7 & \text { Summary and perspectives } & 103\end{array}$

A The multi-temperature black body model in XSPEC 109

B Soft X-ray standard stars $\quad 113$

$\begin{array}{ll}\text { Bibliography } & 121\end{array}$

Acknowledgments 129

$\begin{array}{ll}\text { Curriculum vitae } & 131\end{array}$ 


\section{LIST OF OBJECTS}

AE Aqr $\ldots \ldots \ldots \ldots \ldots \ldots \ldots \ldots \ldots$

HU Aqr . . . . . . . . . . . . . . . . . . . 83

BY Cam . . . . . . . . . . . . . . . . . . . . . 102

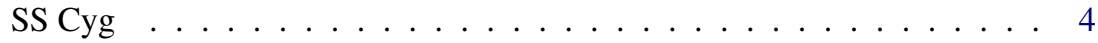

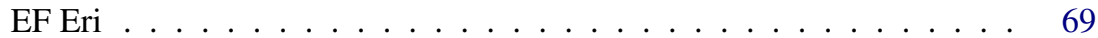

$1 \mathrm{ES} 0102-72.2 \ldots \ldots \ldots \ldots \ldots \ldots$

UZ For . . . . . . . . . . . . . . . . . . . . 100, 101

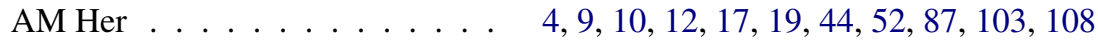

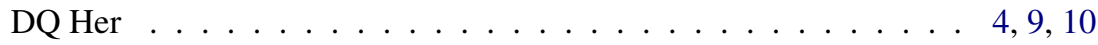

EX Нуа . . . . . . . . . . . . . . . . . . . . . . 4

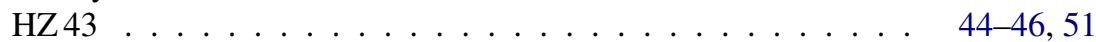

HZ43 A . . . . . . . . . . . . 44-46, 51, 114, 116, 118

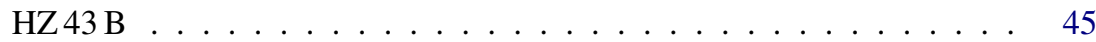

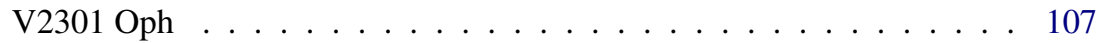

V1309 Ori . . . . . . . . . . . . . . . . . . . 75, 100, 107

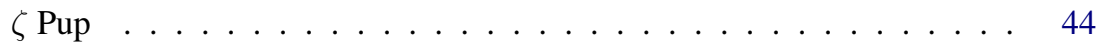

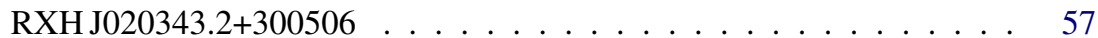

RXJ1856.4-3754 . . . . . . . . . . . . . . . . . 44-51, 104, 113-119

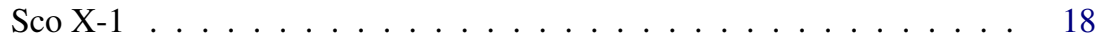

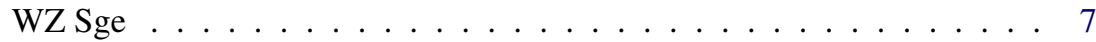

QS Tel . . . . . 3 3, 29, 48, 86-89, 91, 92, 94-96, 98-101, 103, 105-107 AI Tri . . . 3, 29, 48, 52-55, 58-62, 64-70, 72, 75, 79, 82-85, 94, 103-107 
EU UMa . . . . . . . . . . . . . . . . . . . . . . . . . . . . . . 107

EV UMa . . . . . . . . . . . . . . . . . . . . . . . . . . . . . 107

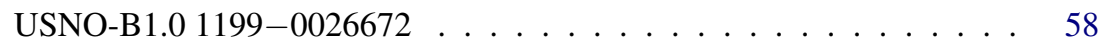

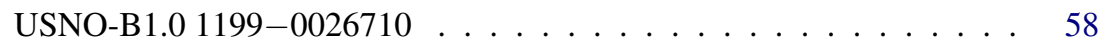

$3 \mathrm{U} 1809+50 \ldots \ldots \ldots \ldots \ldots \ldots \ldots$

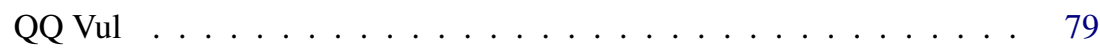

2XMMp J131223.4+173659 . . . . . . . . . . . . . . . . 107 


\section{LIST OF FIGURES}

2.1 Roche geometry . . . . . . . . . . . . . 5

2.2 CV classes . . . . . . . . . . . . . . . . . 10

2.3 Orbital period distribution of cataclysmic variables . . . . . 11

2.4 Column accretion . . . . . . . . . . . . . . . . 13

2.5 Generally used nomenclature in the geometry of polars . . . . 14

2.6 Spectral energy distribution of AM Her . . . . . . . . . . . 17

2.7 Blobby accretion scenario . . . . . . . . . . . . 20

3.1 Main radiation processes in MCVs . . . . . . . . . . . 23

3.2 A 15-temperature black body model . . . . . . . . . . 28

3.3 Temperature structure in the post-shock flow . . . . . . . . 30

3.4 Single- and multi-temperature MEKAL models . . . . . . . . 30

4.1 Schematic view of Wolter telescopes . . . . . . . . . . . 34

4.2 Sketch of the XMM-Newton payload . . . . . . . . . . . 35

4.3 Net effective area and energy bands of EPIC and RGS . . . . 36

4.4 Light path in two of the XMM-Newton telescopes . . . . . . . 37

4.5 Effects of soft proton flares on the EPIC/pn spectra and vignetting ........................ 39

4.6 Selection of EPIC event patterns . . . . . . . . . . . . . 41

4.7 Cut through the X-ray telescope XRT on-board ROSAT . . . . 42

4.8 Flux stability of the RX J1856.4-3754 EPIC/pn spectra . . . . 47

4.9 Archival RX J1856.4-3754 EPIC/MOS2 spectra . . . . . . . 49

5.1 Background and source light curves on August 22, 2005 . . . 55

5.2 XMM-Newton radiation monitor curves . . . . . . . . . 56

5.3 ROSAT and XMM-Newton X-ray light curves of AI Tri . . . . 60

5.4 X-ray, UV, and optical light curves of AI Tri . . . . . . . . 61

5.5 Short-term variability in the EPIC/pn light curve of AI Tri . . . 62

5.6 Optical flickering of AI Tri . . . . . . . . . . . . . 64 
5.7 Periodogram of the optical short-term variability in AI Tri . . . 64

5.8 'Irregular mode' light curves of AI Tri . . . . . . . . . . . . 66

5.9 Comparison of single and multi-temperature black body models 70

5.10 Single and multi-temperature plasma models . . . . . . . . 70

5.11 EPIC spectra of AI Tri compared to the best-fit XSPEC model . 73

5.12 AI Tri EPIC/pn spectra from both the XMM-Newton pointings 74

5.13 Spectral fit to the EPIC and RGS spectra on August 15, 2005 . 75

5.14 Sketch of the binary geometry of AI Tri . . . . . . . . . 77

5.15 Illustration of a non-eclipsing polar with stream dip . . . . . . 79

5.16 Illustration of a stream eclipse . . . . . . . . . . 82

6.1 Radial velocity variations of the QS Tel $\mathrm{H} \beta \lambda 4861 \AA$ line . . . 89

$6.2 \mathrm{EPIC} / \mathrm{pn}$ light curves of QS Tel . . . . . . . . . . . . . 91

$6.3 \mathrm{EPIC} / \mathrm{pn}$ light curves of QS Tel during the bright phases . . . . 92

6.4 Optical and UV light curves of QS Tel . . . . . . . . . . . 93

6.5 EPIC spectra of QS Tel compared to the best-fit XSPEC model 96

6.6 Multi-temperature fits to the EPIC/pn spectrum of QS Tel . . . 96

6.7 EPIC/pn spectra of QS Tel during the bright and the faint phases 98

6.8 Flaring structure of the soft band light curve . . . . . . . 100

A.1 Parametrization of the Gaussian temperature distribution . . 110

B.1 Archival EPIC/pn spectrum of RX J1856.4-3754 . . . . . 115

B.2 Archival EPIC/MOS1 spectrum of RX J1856.4-3754 . . . . 117

B.3 Archival EPIC/MOS2 spectrum of RX J1856.4-3754 . . . . . 119 


\section{LIST OF TABLES}

3.1 Temperatures, normalizations, and flux contributions for an example multi black body model . . . . . . . . . . . 28

5.1 Log of the ROSAT and XMM-Newton observations and of the optical photometry of AI Tri . . . . . . . . . . . . . 54

5.2 Heliocentric timings of the $V$-band minima of AI Tri . . . . . 63

5.3 Results of the best XSPEC fits to the total and to the phaseresolved EPIC spectra of AI Tri . . . . . . . . . . . . . . 68

5.4 Hardness ratios and unabsorbed model fluxes for the phaseresolved AI Tri spectra . . . . . . . . . . . . . . 84

6.1 Log of the XMM-Newton and optical observations of QS Tel . $\quad 88$

6.2 Results of the XSPEC models to the total and to the phaseresolved EPIC spectra of QS Tel . . . . . . . . . . . . 95

B.1 Archival XMM-Newton EPIC/pn observations of HZ43 A and RX J1856.4-3754 . . . . . . . . . . . . . . . . . . . . 114

B.2 Archival XMM-Newton EPIC/MOS1 observations of HZ43 A

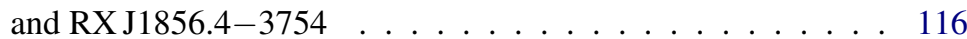

B.3 Archival XMM-Newton EPIC/MOS2 observations of HZ43 A

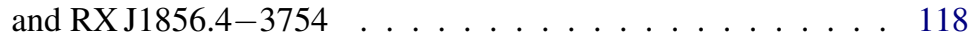




\section{CHAPTER 1}

\section{Introduction}

In the context of a comprehensive knowledge on star formation and stellar evolution, binary and multiple stars play an important role. Eclipsing binaries for example allow for a direct derivation of crucial system parameters like masses and radii and, hence, can serve as empirical test cases for theoretical models. The majority of stars are hosted in multiple systems, orbiting around their common center of mass. The components may be very similar to each other, or may represent as different evolutionary stages as a white dwarf or a neutron star, at the end of its active "life", plus a relatively young object. Throughout their evolution, they are expected to interact gravitationally and radiatively. Understanding these processes is essential to explain observed phenomena like the brightness variations of cataclysmic variables, eruptive novae, or a subclass of supernovae, the type Ia. The interaction is most intense in close systems, close enough that some of them would even fit between the Earth and its Moon or into the Sun. In this work, one group of close interacting binaries is studied, cataclysmic variables with a strong magnetic field.

Magnetic cataclysmic variables of AM Her type (MCVs), also called polars, consist of an accreting magnetic white dwarf, accompanied by a low-mass secondary which fills its Roche lobe, i. e. its maximum possible extent within the binary configuration. In a 'Roche-lobe overflow', the secondary donates mass into the gravitational potential of the white dwarf. Due to a strong magnetic field ( $B \sim 7-230 \mathrm{MG}$ ) of the primary, this accretion stream couples to the magnetic field lines and reaches the white dwarf in the immediate vicinity of one or both of its magnetic poles. The accreted material, decelerated above the white-dwarf surface and heated to about $10^{8} \mathrm{~K}$, forms a strong shock in the flow. Within the accretion column below the shock, it cools by the emission of optical cyclotron radiation and of hard X-rays, which are reprocessed 
in the white-dwarf photosphere, then seen in the soft X-ray energy regime $(E \lesssim 0.5 \mathrm{keV})$ and in the ultraviolet. Correspondingly, the X-ray spectra of most MCVs are characterized by two main spectral components: at energies above about $0.5 \mathrm{keV}$ by hard thermal bremsstrahlung-like emission; at lower energies by quasi-black body radiation. According to the standard accretion model, the soft flux that is re-emitted from the region around the white dwarf's magnetic pole approximately balances the hard emission. In several systems, however, the soft X-ray flux unexpectedly excels the hard component. This 'soft X-ray excess' is meanwhile believed to develop, when cyclotron emission becomes the main cooling process at a high magnetic field strength of the white dwarf. The material in the accretion stream of these systems resolves into dense filaments, inducing inhomogeneous and time-variable accretion from the post-shock flow onto the white dwarf. The 'blobby' accretion events give rise to high variability in the X-ray light curves, typically most pronounced in the soft X-ray regime. The actual fraction of soft X-ray dominated systems in the polars is still under discussion. First analyses of the soft-to-hard flux ratios in polars were performed on the basis of spectra obtained with the X-ray satellite ROSAT. Due to the limited energy range of $0.1-2.4 \mathrm{keV}$, the hard emission component usually has been described by a bremsstrahlung model, if necessary fixing the temperature to what was considered a realistic value. The structure of the post-shock flow and of the accretion region, however, is more complex, comprising a wide range of temperatures and densities. The higher spectral resolution and larger energy coverage of the detectors on-board the current X-ray satellite missions Chandra and XMM-Newton have allowed for employment of plasma models. The choice of the model components in the spectral fits has a noticeable influence on the soft-to-hard ratios, which characterize the degree of a potential dominance of soft X-ray emission. Plasma model spectra, the shape of which is mostly shallower than a bremsstrahlung spectrum, may reduce the ratio values and the number of polars for that a soft X-ray excess can be stated. In the last decade, yet a small group of systems without a detectable soft X-ray component have been confirmed. Their reprocessed component possibly is cooler and shifted towards longer wavelengths, out of the energy range which is covered by the X-ray detectors.

In the context of the multifarious picture of the accretion processes and the emission measured from polars, we study selected systems with XMM-Newton. They are known to show a distinct soft X-ray flux and have not been subject to high-resolution X-ray observations before. Optical spectroscopy and photometry and archival ROSAT data supplement the recent XMM-Newton 
observations of the two systems AI Tri and QS Tel, on which this work focuses. Main goals are to

$\checkmark$ investigate the contribution of the different X-ray emitting regions to the spectra and light curves,

$\diamond$ derive lower limits for their soft X-ray excess,

- examine the impact of various black body and plasma models on the spectral fits,

- constrain the geometrical properties of the observed objects.

Both systems show high magnetic field strengths. Previous studies have revealed differing system parameters like accretion rates and temperatures for them. AI Tri belongs to the polars with the longest periods measured. For QS Tel, one of the few objects found within the period gap of cataclysmic variables, divers accretion mode changes have been observed. With these characteristics, AI Tri and QS Tel represent heterogeneous examples of soft X-ray dominated polars.

A more detailed introduction into the properties of close binary systems and particularly of magnetic cataclysmic variables provides the following Chapter 2. Methods for modeling the observed X-ray spectra, including new approaches to the complex temperature structure in the X-ray emission regions, are given in Chapter 3. Chapter 4 presents the space-based instruments with which the observational X-ray data were obtained. Pointing out the importance and complexity of a thorough knowledge of the instrumental properties, the XMM-Newton calibration in the soft X-ray energy range is investigated, fundamental in the analysis of magnetic cataclysmic variables. Chapter 5 and Chapter 6 comprise the spectroscopic and photometric analyses of the two observed objects, AI Tri and QS Tel, respectively. The results are summarized and discussed in Chapter 7. 


\section{CHAPTER 2}

\section{Magnetic cataclysmic variables in X-rays}

Stars of unknown nature, showing strong brightness variations on time scales from decades down to seconds, early attracted the astronomers' attention. Initially subsumed under the name "Novae" ("new stars", incorporating the supernovae at that time), they have later on also been termed cataclysmic (eruptive) variable stars (CVs) due to the calamitous event which was suspected to trigger the observed luminosity eruptions. With AE Aqr (Joy 1954), DQ Her (Walker 1954), and SS Cyg (Joy 1956), several CVs were identified as binary systems in the 1950s. In the first part of a twelve-paper series, Kraft (1962) presented three fundamental and observationally supported hypotheses on the nature of cataclysmic variables: they are short-period binaries; one component appears to fill its Roche lobe, initializing mass transfer within the system; the accretor most probably is a white dwarf. These characteristics meanwhile constitute the definition of the CV class. Within the UHURU sky survey, X-ray emission from CVs was discovered; for the first time in the case of EX Hya (Warner 1972). One of the CVs, AM Her, to which Berg \& Duthie (1977) identified the UHURU source $3 \mathrm{U} 1809+50$ as X-ray counterpart, appeared to differ in its properties from the other known members of the class. Tapia (1977) detected variable linear and circular polarization in the optical spectra, indicating a strong magnetic field of the compact component. Subsequently, Chanmugam \& Wagner (1977) presented the physical explanation in the form of a synchronously rotating binary system comprising an accreting magnetic white dwarf. With respect to the high degree of optical polarization, the sub-class of AM Her-type cataclysmic variables also has been called polars, adopting a suggestion of Krzeminski \& Serkowski (1977). The following Sections give an introduction to magnetic cataclysmic variables (MCVs). Comprehensive overviews are provided by Warner (2003) and Cropper (1990). 

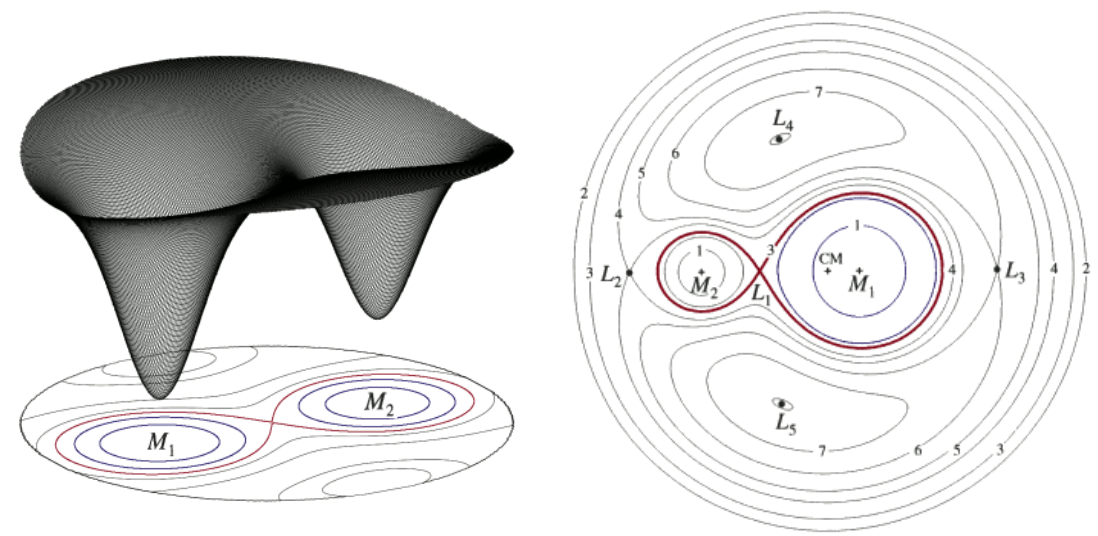

Figure 2.1: Roche geometry: equipotential surfaces in the orbital plane of a binary. The Roche lobes of the system components are marked in red, $L_{1}$ to $L_{5}$ are the Lagrangian points of equilibrium. Left panel adapted from Motl (2001), right panel from Frank et al. (2002).

\subsection{Close binary systems}

Close binaries usually interact physically, in addition to the effects of the stars' individual gravitational potentials. This interaction may be for instance the tidal deformation or the irradiative surface heating of a component. Shortperiod systems exhibit nearly circular orbits, to which Kepler's third law in its generalized Newtonian form applies:

$$
a^{3}=\frac{G}{4 \pi^{2}}\left(M_{1}+M_{2}\right) P_{\text {orb }}^{2},
$$

where $a$ is the binary separation between the centers of mass, $G$ the gravitational constant, and $M_{i}$ the mass of the $i$ th component. In compact binaries, the centrifugal force counteracts the gravitational forces of the individual stars. The Roche potential, formulated by Édouard Roche $(1820-1883)$, connects the associated potentials in the co-rotating frame:

$$
\Phi_{\text {Roche }}(\boldsymbol{r})=-\frac{G M_{1}}{\left|\boldsymbol{r}-\boldsymbol{r}_{\mathbf{1}}\right|}-\frac{G M_{2}}{\left|\boldsymbol{r}-\boldsymbol{r}_{\mathbf{2}}\right|}-\frac{1}{2}(\boldsymbol{\omega} \times \boldsymbol{r})^{2} .
$$


$\boldsymbol{\omega}$ is the angular velocity of the system, $\boldsymbol{r}$ the radial distance from the center of mass, and $\boldsymbol{r}_{\boldsymbol{i}}$ the positions of the stars, which are substituted by point masses (Frank et al. 2002). Figure 2.1 shows the equipotential surfaces in the binary Roche geometry. Their contours depend on the mass ratio, while the total dimensions scale with the binary separation. The Roche lobe (also called inner Lagrangian surface), the largest of the closed equipotentials, defines the maximum radial extent of each star, its Roche volume. The radius of the secondary's Roche lobe can be approximated using a formula from Eggleton (1983),

$$
R_{2}=\frac{0.49 q^{2 / 3}}{0.6 q^{2 / 3}+\ln \left(1+q^{1 / 3}\right)},
$$

with an accuracy better than $1 \% ; q=M_{2} / M_{1}$ represents the mass ratio of the two stars, the more massive one of which is defined as the primary component. Inside the Roche volumes, the gravitation force dominates, while the Coriolis force governs the outer regions. Five points of stable and unstable equilibrium characterize the Roche geometry, the Lagrangian points $L_{1}$ to $L_{5}$ (right panel in Figure 2.1). The stars' surfaces follow the shapes of the Roche equipotential surfaces, along which a binary component thus is distorted in case it reaches a high Roche filling factor. According to the nomenclature introduced by Kopal (1955), a system in which both the stars completely fill their Roche lobes is called a contact binary. The opposite case is the detached system, in which both components lie well within the Roche volumes. In semi-detached binaries like the cataclysmic variables, but one of the two components fills its side of the Roche lobe.

\subsection{Evolution of compact binaries}

A star may get in contact with its Roche lobe either by expansion of the star itself or by shrinking of the Roche lobe. Both alternatives occur during the evolution of semi-detached binaries. Although still under discussion in many aspects, a largely accepted evolutionary scenario has been developed during the last decades. The systems commence as wide binaries with large orbital periods - wide enough that the two components will not merge during their further evolution. ${ }^{1}$ Typical dimensions are thus separations larger than the radius of

\footnotetext{
1 The longest and most stable stage in the evolution of a star is the period in which nuclear fusion of hydrogen into helium takes place in the stellar core. The hydrogen burning stars settle the
} 
a red giant between fifty and several thousand solar radii, and orbital periods from ten days up to ten years and more. The initial main sequence mass of the primaries in cataclysmic variables, hosting a white dwarf, ranges between 0.95 and 9 solar masses (de Kool 1992), allowing the star to evolve within the galactic time scale and to drop below the Chandrasekhar mass limit (Equation 2.4) during the post-main sequence stages. The primary, more massive component passes the zero-age main sequence faster than the low-mass secondary. Following the standard evolution scenario of single stars (cf. Kippenhahn \& Weigert 1990), it expands to a red giant after exploiting its hydrogen supply. Thus the primary reaches the boundaries of the Roche lobe, starting dynamically unstable mass transfer onto the secondary companion via the inner Lagrangian point $L_{1}$ (Figure 2.1). This implies high mass flow rates on dynamical time scales, roughly on the order of orbital periods. The secondary cannot subsume the large amount of overflowing material, and a common envelope forms around the two stellar cores. Frictional braking releases on the one hand angular momentum from the system, whose separation shrinks in consequence, and on the other hand energy by heating the envelope. As soon as the binding energy is surpassed by the released frictional energy in the spiral-in process, the system loses its envelope (Paczyński 1976). The remaining detached binary is also referred to as a pre-cataclysmic variable.

Two processes can be responsible for the following contraction of the system: gravitational radiation and magnetic braking. The very effective mechanism of magnetic braking (Verbunt \& Zwaan 1981) relates to the interaction between the stellar wind and the magnetic field of a star. The magnetically coupled particles in the stellar wind, drifting outwards, withdraw angular momentum from the star (Hameury et al. 1988). Due to the large extent of the region in which the interaction takes place and the accordingly long lever arm, the braking is efficient yet for a low magnetic field strength and moderate stellar wind. Gravitational radiation, emerging when massive bodies accelerate, in particular becomes important at short orbital periods and small binary separations. As discussed by Kraft et al. (1962) on the example of WZ Sge, it reduces the angular momentum of the system in dependence on the component masses and separation, slowly driving the system towards shorter periods in conse-

(zero-age) main sequence in the Hertzsprung-Russell (or temperature-luminosity) diagram. When the inner hydrogen fuel is exhausted, the core becomes surrounded by a hydrogen burning shell, and the star evolves into a red giant. During the giant phase, a star reaches its maximum extent. A carbon-oxygen white dwarf is the final evolutionary stage of a solar-like star. The burning processes have ceased, the stellar core consists of degenerate electron gas. 
quence. Abating the geometrical dimensions in a binary system, gravitational and magnetic braking let the secondary attach its Roche lobe, and dynamically and thermally stable, conservative mass transfer onto the primary commences.

\subsection{The configuration of cataclysmic variables}

Cataclysmic variables (CVs) are close interacting binaries which comprise a white dwarf primary and a late-type main sequence secondary. As the secondary fills its Roche lobe (Section 2.1), material enters the gravitational potential well of the primary via the saddle point $L_{1}$ in a Roche lobe overflow. Knowing the orbital periods on the order of hours and the components' masses in cataclysmic variables, the typical binary separation can be estimated to a few times the Earth-moon distance.

The white dwarf primary represents the final evolutionary stage of a lowto medium-mass star which has lost a sufficient amount of mass to fall below Chandrasekhar's limiting mass

$$
M_{\mathrm{Ch}}=\frac{4}{\mu_{\mathrm{e}}^{2}} \cdot 1.459 \mathrm{M}_{\odot} \simeq 1.44 \mathrm{M}_{\odot}
$$

(Kippenhahn \& Weigert 1990). $\mu_{\mathrm{e}}$ is the mass of a free electron in atomic mass units, $\mathrm{M}_{\odot}$ the mass of the Sun. The usual extent $\mathrm{R}_{\mathrm{WD}}=10^{9}-10^{10} \mathrm{~cm}$ of a white dwarf is comparable to the Earth radius. ${ }^{2}$ With a surface temperature ${ }^{3}$ typically on the order of several ten thousand degrees, its photosphere mostly emits from the blue optical down to the UV and FUV wavelength band, while the flux maximum of the accretion heated surface area lies in the soft X-ray or EUV regime at about $10^{6} \mathrm{~K}$. The light curves are modulated due to the revolution of the accretion region with the orbital cycle. The process of accretion onto the mass-capturing white dwarf dominates the high-energy emission at X-ray wavelengths. The accretion luminosity

$$
L_{\mathrm{accr}}=\frac{G \mathrm{M}_{\mathrm{WD}} \dot{\mathrm{M}}}{\mathrm{R}_{\mathrm{WD}}}
$$

reaches for characteristic white-dwarf masses $\mathrm{M}_{W D}=0.5-1.3 \mathrm{M}_{\odot}$, radii $\mathrm{R}_{\mathrm{WD}}=5 \cdot 10^{-3}-10^{-2} \mathrm{R}_{\odot}$, and accretion rates $\dot{M}=10^{-11}-10^{-8} \mathrm{M}_{\odot} \mathrm{yr}^{-1}$ values on the order of $L_{\text {accr }}=0.1-1000 \mathrm{~L}_{\odot} \simeq 10^{32}-10^{36} \mathrm{erg} \mathrm{s}^{-1}$.

\footnotetext{
${ }^{2}$ In astrophysics, cgs units are commonly used in addition to the SI system.

${ }^{3}$ The surface temperature that corresponds to a black body of the same total flux density as the star is called its effective temperature $T_{\text {eff }}$.
} 
The Roche-lobe filling and mass donating secondary is a main sequence star with a spectral type ${ }^{4}$ between $\mathrm{K}$ and $\mathrm{M}$, apart from a few systems with late $\mathrm{G}$ donors (Knigge 2006). It is thus primarily seen in the red and infrared. The distortion along the boundaries of the equipotential surface leads to ellipsoidal light curve variations with the orbital cycle, superposed by the signature from the revolving irradiated part of the surface. Representative surface magnetic field strengths of this class range on the order of $B=10^{2}-10^{3} \mathrm{G}$. Analogously to single stars of similar spectral type, the secondary is expected to show a considerable number of star spots due to the fast rotation.

The third principal constituent of a cataclysmic variable is the accretion stream, connecting the low-mass secondary and the white dwarf, and seen in the optical and ultraviolet band. The matter starts near the inner Lagrangian point $L_{1}$ at approximately the local speed of sound $\left(c_{\mathrm{s}} \lesssim 10 \mathrm{~km} / \mathrm{s}\right.$, Frank et al. 2002) and falls towards the primary. The fast rotation of the system governs the path of the highly collimated stream, which follows roughly a single particle trajectory. Under the Coriolis force due to the angular momentum, it is deflected and accelerated to supersonic velocities. On its way around a nonmagnetic primary, it rejoins itself and - deviating from a pure Keplerian orbit - widens to an accretion disk under the influence of viscosity. From the inner edge of the disk, the matter falls onto the equatorial regions of the white dwarf. If the primary features a strong magnetic field ( $B \gtrsim 7 \mathrm{MG}$ ), the magnetic pressure exceeds the ram pressure in the vicinity of the white dwarf. The accretion stream then couples to the magnetic field lines, preventing the formation of a disk and leading the accretion stream towards the magnetic poles of the primary. Spin period of the white dwarf and orbital period are synchronized. These systems are called polars or AM Her type CVs after their prototype (more details in Section 2.4). In intermediate polars (IP) or DQ Her type CVs with moderate field strengths between about 1 and $10 \mathrm{MG}$, the outer regions of an accretion disk can establish. The magnetic field, however, disrupts the inner part of the disk and forces the matter into an accretion stream along the field lines. The primary in intermediate polars rotates freely. The theoretical concept that IPs may evolve into polars (e. g. Chanmugam \& Ray 1984; Webbink \& Wickramasinghe 2002) has recently been observationally sustained by Schwarz et al. (2007) on the polar Paloma. Figure 2.2 displays a sketch of the three classes of cataclysmic variables.

\footnotetext{
${ }^{4}$ Main-sequence stars are classified according to their spectral characteristics ("color") and thus their surface temperature. $\mathrm{M}$ and $\mathrm{K}$ stars with effective temperatures below $T_{\text {eff }} \simeq 5000 \mathrm{~K}$ range among the coolest ones; the Sun is of spectral type G.
} 

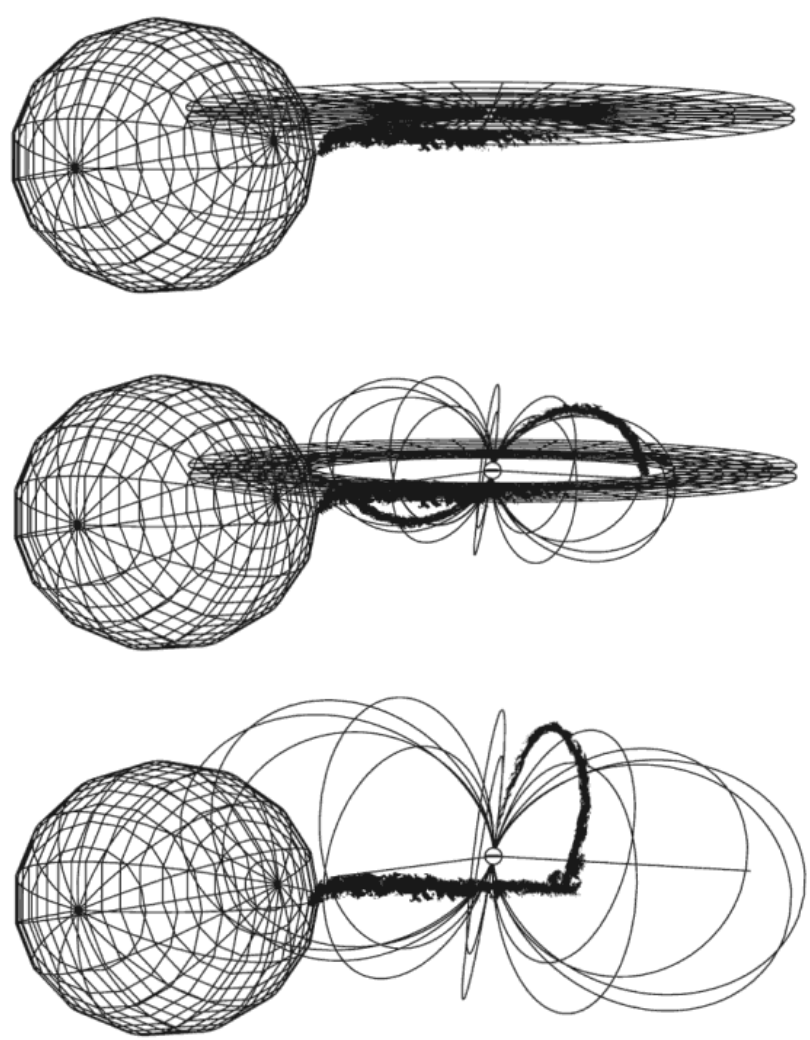

Figure 2.2: Schematic view of the three classes of cataclysmic variables. The low-mass secondary (left) donates matter to the compact primary (right). Top: Non-magnetic cataclysmic variable. The accreted matter forms a disk around the white dwarf. Center: Intermediate polar (DQ Her type). The moderate magnetic field of the primary disrupts the accretion disk. Bottom: Polar (AM Her type). The ionized material follows the magnetic field lines. From Cropper et al. (2002).

The orbital periods of cataclysmic variables are primarily distributed between eighty minutes and twelve hours with a pronounced gap between two and three hours (Figure 2.3). Below the short-period cutoff, the small secondary's Roche lobe can only host a degenerate object, which expands as a response to the mass loss, thus increasing the orbital period and defining its 


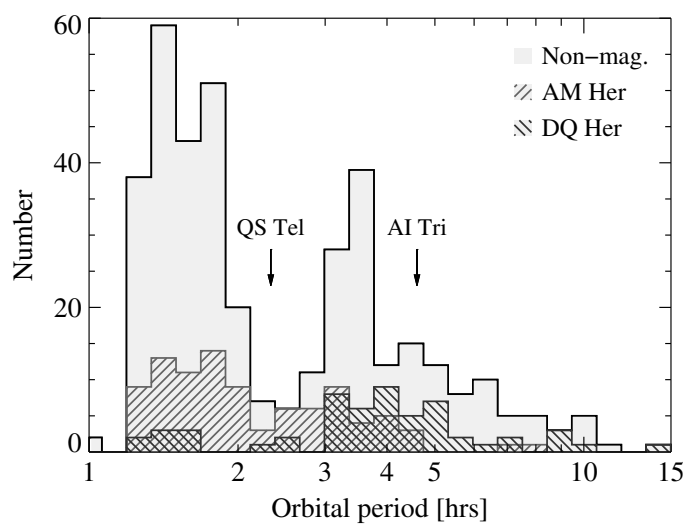

Figure 2.3: Orbital period distribution of cataclysmic variables. The data have been adapted from the Ritter \& Kolb (2003) catalog in the 2008 July 1 online version (http://physics.open.ac.uk/RKcat).

minimum. Above the long-period cutoff, the secondary loses contact to its Roche lobe, and the accretion process ceases (King 1988). The observational lack of CVs with periods in-between two and three hours challenges theory. The magnetic braking mechanism (Section 2.2) is predicted to conclude at orbital periods around three hours, when the secondary becomes fully convective, either due to a rearrangement of the surface magnetic field topology (Taam \& Spruit 1989), or because of a drop in stellar activity (Rappaport et al. 1983 and Spruit \& Ritter 1983, independently). The secondary shrinks to an extent smaller than its Roche volume, switching off the mass transfer process. Angular momentum losses caused by gravitational radiation drive the system to shorter periods, reinstating mass transfer around $P_{\text {orb }} \simeq 2$ hrs. In cataclysmic variables with periods above $P_{\text {orb }} \gtrsim 3 \mathrm{hrs}$, the magnetic braking overruns the effects of gravitational radiation in extracting angular momentum from the system, whereas below only gravitational radiation is active. Mass transfer rates above the period gap are generally higher than below.

Polars show a less pronounced period gap than non-magnetic cataclysmic variables (see Figure 2.3). Webbink \& Wickramasinghe (2002) state that the white-dwarf magnetosphere possibly confines the stellar wind from the secondary, shortening the lever arm of the wind-field interaction. The subsequent reduction of the magnetic braking effect in all magnetic CVs may explain the narrower period gap observed. 


\subsection{The influence of the magnetic field: Polars}

Since Tapia (1977) for the first time denoted the existence of a magnetic field in AM Her, about $25 \%$ of the cataclysmic variables have been revealed to host magnetic white dwarfs. Two thirds of them exhibit field strengths higher than $B \gtrsim 10 \mathrm{MG}$, at which no accretion disk forms, and are thus classified as polars (Ritter \& Kolb 2003, catalog edition 7.10, July 2008). Some major features like the synchronization of the system and modifications of the accretion process, the emitted radiation, and the period distribution (see Section 2.3) rely on the influence of the magnetic field and shall be summarized in the following.

The origin of the strong magnetic fields is still under discussion. One possible explanation are initially low fossil fields, remnants from the main sequence or post-main sequence evolutionary stage (Wickramasinghe \& Ferrario 2005). When the star contracts to a white dwarf, they intensify, conserving the total magnetic flux. Alternatively, the magnetic field may emerge during the common envelope phase from a stellar dynamo driven by convection and differential rotation (Tout et al. 2008). While a dipole usually is an appropriate approximation to the field far from the white dwarf, its structure close to the surface may be complex (Euchner et al. 2002).

Interaction between the fields of the primary and the secondary components synchronizes the orbital and the spin period of the white dwarf in most of the polars (Campbell \& Schwope 1999). When the magnetic torque clearly dominates the accretion torque with

$$
\frac{\mu_{1} \mu_{2}}{a^{3}}>\dot{M} \omega d^{2}
$$

the synchronization of the system gets locked (Campbell 1985). $\mu_{1}$ and $\mu_{2}$ are the magnetic moments of the stars, $a$ is their separation, $\dot{M}$ the accretion rate onto the primary, $\omega=2 \pi / P_{\text {orb }}$ the orbital angular velocity, and $d$ the distance between the inner Lagrangian point $L_{1}$ and the center of the white dwarf. The rotation axis of the white dwarf is perpendicular to the orbital plane then.

The magnetic field strongly influences the accretion process. The ionized matter in the accretion stream attaches to the field lines within the threading region. This coupling takes place around the magnetospheric or Alfvén radius, where the magnetic pressure in the stream balances the ram pressure (Liebert \& Stockman 1985):

$$
\frac{B^{2}}{8 \pi}=\varrho v^{2}=\frac{\dot{M}}{\pi \sigma^{2} v} v^{2}
$$




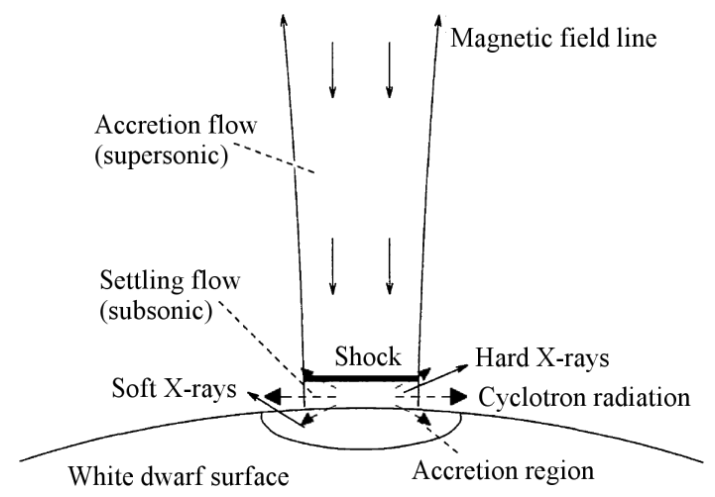

Figure 2.4: Column accretion onto a magnetic white dwarf. In this simplified view, the material reaches the surface of the white dwarf along the magnetic field lines in a nearly radial symmetric accretion column. In the flow below the shock, it decelerates and cools. Adapted from van Teeseling et al. (1994).

The location of the threading region thus depends on the magnetic field strength $B$, the mass accretion rate $\dot{M}$, the cross section $\sigma$ of the stream, and the stream velocity $v$, which is close to the free fall velocity

$$
v_{\mathrm{ff}}=\sqrt{\frac{2 G \mathrm{M}_{\mathrm{WD}}}{\mathrm{R}_{\mathrm{WD}}}} .
$$

In particular, it changes with the mass transfer rate $\dot{M}$ during different states of accretion. Passing the threading region, the accretion stream is lifted off the orbital plane and reaches the white dwarf along the field lines in the immediate vicinity of its magnetic poles (lowest panel in Figure 2.2). The accreted material has to be decelerated in the accretion column above the white-dwarf surface to subsonic velocities. The predominant cooling processes are radiative cooling, mainly by free-free emission (bremsstrahlung), cyclotron emission from the electrons gyrating around the field lines, and Compton scattering of reprocessed electrons from the white-dwarf surface. In the simplified standard picture of the accretion column as illustrated in Figure 2.4, a strong shock in the flow forms at characteristic temperatures of

$$
T_{\mathrm{sh}}=\frac{3}{8} \frac{G \mathrm{M}_{\mathrm{WD}} \mu \mathrm{m}_{\mathrm{H}}}{k \mathrm{R}_{\mathrm{WD}}}
$$




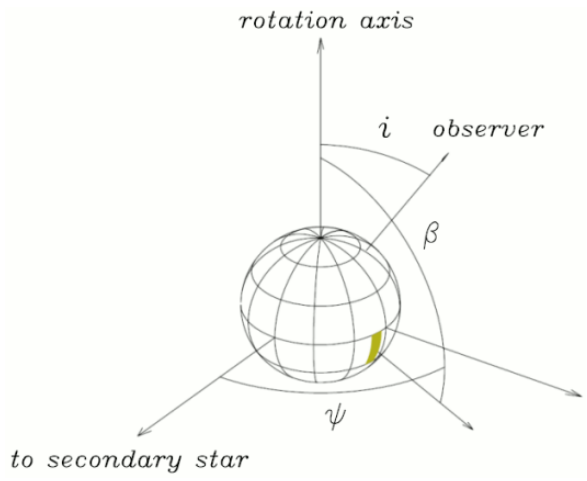

Figure 2.5: Generally used nomenclature in the geometry of polars. The accretion spot is located at the colatitude $\beta$, the angular distance to the rotation axis, and at the azimuth $\psi$, the angular distance to the connecting line between primary and secondary. The angle between the rotation axis and the observer's line of sight defines the inclination $i$. Adapted from Schwarz et al. (2001).

where $\mu$ is the mean molecular weight, $m_{\mathrm{H}}$ the hydrogen atom mass, $k$ the Boltzmann constant, and $G$ again the gravitational constant (Frank et al. 2002). Hence, the matter is heated to typical temperatures on the order of $10^{8} \mathrm{~K}$. At low local mass flow densities ${ }^{5} \dot{m} \lesssim 0.1 \mathrm{~g} \mathrm{~cm}^{-2} \mathrm{~s}^{-1}$, the plasma temperature stays well below the value given in Equation 2.9, and the material reaches the white dwarf without developing a shock (Woelk \& Beuermann 1992). Rousseau et al. (1996) expose a wide range of mass flow densities and a core-halo structure of the accretion region in this bombardment solution. The accretion region on the white-dwarf surface is typically oriented at small azimuthal angles $\psi$ towards the secondary (Cropper 1988). Figure 2.5 shows the general geometry of the primary in polars.

Erratic long-term changes in the brightness of polars - up to several magnitudes in the optical band - are directly connected to variations in the mass transfer rate and referred to as high, intermediate, and low states of accretion. As possible reasons for the changes between these states, King \& Lasota (1984) discuss the effects of irradiation feedback, which enhance the mass transfer due to the heating of the secondary surface and cause it to drop when the heated material is depleted. Observational results for instance by Hessman et al. (2000)

\footnotetext{
${ }^{5}$ The local mass flow density $\dot{m}$ is the (local) accretion rate per unit area.
} 
and Kafka \& Honeycutt (2005) support the star-spot concept of Livio \& Pringle (1994). Spots, originating from stellar activity and the fast rotation, lower the mass transfer rate as they pass the vicinity of the inner Lagrangian point $L_{1}$ during their motion over the star surface.

The lack of an accretion disk in the highly magnetic systems opens a direct view into the accretion process. The flux maximum of the emitted radiation from the accretion column lies in the hard X-ray regime, whereas the heated surface of the white dwarf is mainly seen in the soft X-ray or UV. The polarized cyclotron radiation turns up in the infrared, optical, and UV band. From the fundamental cyclotron frequency

$$
\omega_{\mathrm{cyc}}=\frac{e B}{m_{\mathrm{e}} c}
$$

with $e$ being the elementary charge, $m_{\mathrm{e}}$ the electron rest mass, and $c$ the speed of light in vacuum, the magnetic field strength $B$ can be derived.

\subsection{X-ray diagnostics of MCVs}

Starting with the first detection of an X-ray source outside the Solar System (Giacconi et al. 1962), X-ray astrophysics has become a major discipline in the exploration of the universe. Almost all kinds of astronomical objects from nearby stars to distant luminous galaxies and their dynamics are subjects to $\mathrm{X}$-ray diagnostics. X-radiation gives a picture of the hot universe, as it emerges in extreme and often explosive processes, in intense magnetic or radiation fields. The astrophysical plasmas seen in X-rays are found at high temperatures and low densities, so that the assumption of local thermodynamic equilibrium does no longer hold in general. The spectral continuum, directly reflecting the X-ray generating processes, typically comprises one or more of four major forms. Black body-like radiation in thermodynamic equilibrium describes the spectral shape of many stellar objects. Electrons in a hot, optically thin gas produce bremsstrahlung when they are accelerated or braked in the field of the plasma ions. Under the additional influence of a magnetic field, the electrons circle around the field lines and emit synchrotron radiation along the direction of their motion at high velocities. The emerging spectrum is close to a power-law. Its non-relativistic analogon is the cyclotron radiation seen at longer wavelengths from the UV up to the infrared. Comptonization involves energy transfer by multiple Compton scattering of high-energy photons in a hot 
ionized plasma and the inverse process, in which soft photons acquire energy from the surrounding gas. The diagnostics of spectral lines and their formation gains importance with the increasing instrumental resolution. Mainly the $\mathrm{K}$ - and L-shell transitions of heavy elements up to iron imprint the spectra in the X-ray band. One of the processes most prominently detectable in X-rays is mass transfer via accretion.

\subsubsection{X-ray emission from polars}

The X-ray emission from polars reflects the accretion processes described in Section 2.4. It is mainly characterized by two components: on the one hand by hard thermal free-free radiation, originating in the optically thin, cooling accretion column; on the other hand by optically thick black body-like radiation from the radiatively and conductively heated surface region around the white dwarf's magnetic pole(s) in the soft X-ray regime. The standard model of accretion in magnetic cataclysmic variables, developed by Lamb \& Masters (1979) and King \& Lasota (1979), predicts that half of the flux from the accretion column is reprocessed in the white-dwarf photosphere, i. e. scattered back or re-emitted as soft X-rays. The X-ray spectrum thus resolves into two distinct parts, intersecting at about $0.5 \mathrm{keV}$ (Figure 2.6). The hard continuum, emerging from free-free emission, is superposed by emission lines from the optically thin accretion plasma. Galactic absorption by the interstellar medium and intrinsic absorption by the partially ionized plasma may additionally influence the spectra.

The orbital revolution of the X-ray emitting constituents modulates the light curves. While the hard X-ray light curve mostly mirrors the changing orientation of the accretion column against the line of sight, the heated white-dwarf surface shapes the orbital variation in the soft component. When the accretion region points towards the observer, a bright phase of the light curve is seen; during a faint phase, the white dwarf may occult the region. Sharp dips in the light curves probably arise, when the accretion stream crosses the line of sight during the orbital cycle, partly eclipsing the accretion region. Photo-electric absorption in the X-ray regime and free-free absorption in the infrared and optical then cause the observed flux to drop for about a tenth of the orbital cycle. Substructure on time scales of seconds and minutes in the optical and X-ray bands may arise when thermal instabilities excite quasi-periodic oscillations (QPOs) in the height of the shock front above the white-dwarf surface (Langer et al. 1981). According to Chanmugam et al. (1985), the QPOs weaken in highly magnetic systems in which the cyclotron cooling exceeds the bremsstrahlung. 


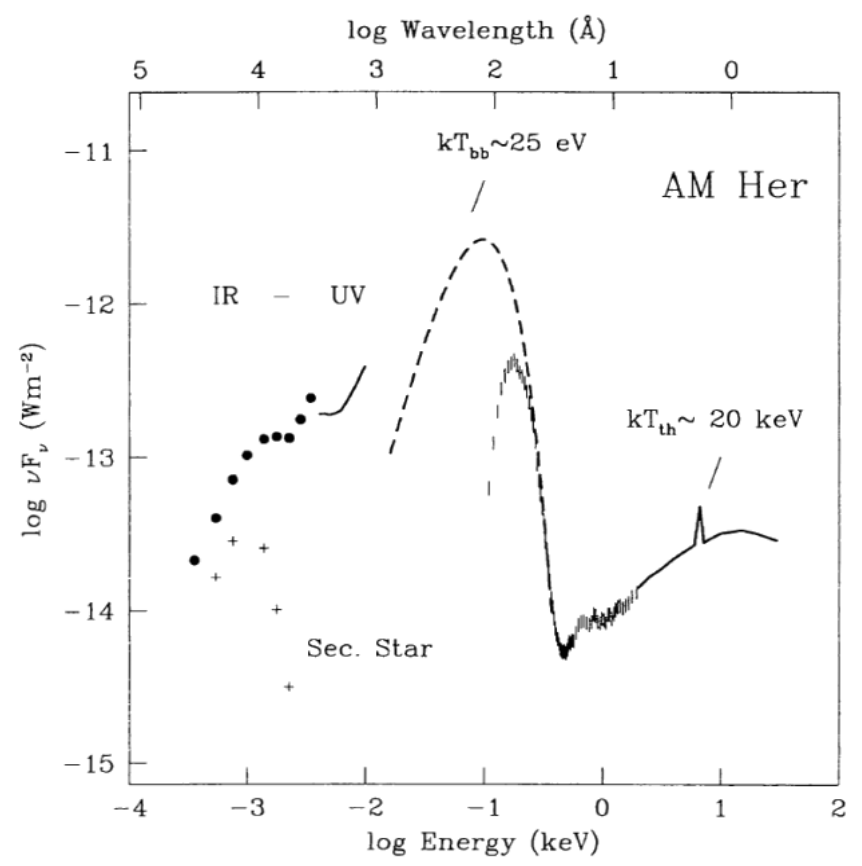

Figure 2.6: Spectral energy distribution of AM Her. The dominant components are cyclotron emission in the infrared and optical, UV flux from the heated white-dwarf surface and from the accretion stream (IUE data), a soft X-ray quasi-black body arising from the accretion region (ROSAT data of April 1991), and hard X-ray emission produced in the accretion column (HEAO-1 data). The unabsorbed black body model flux corresponding to the soft $\mathrm{X}$-ray component is marked as a dashed line in addition. From Beuermann (1999).

\subsubsection{Hydrodynamic models}

Both in the shock front case at high and in the bombardment case at low mass flow rates $\left(\dot{m} \lesssim 0.1 \mathrm{~g} \mathrm{~cm}^{-2} \mathrm{~s}^{-1}\right)$, the X-ray emitting region of the accretion column is highly variable in time and space and highly structured. Temperature, velocity, density, pressure, and mass flow density follow different distributions. In order to describe the X-ray emission, it is necessary to treat hydrodynamics and radiative transfer with respect to the geometrical properties. This concerns for instance the interaction between regions of different physical conditions or 
the influence of gravitation. Gravity effects depend on the shock height and are thus most important in systems with tall shocks, least important in the case of strong cyclotron cooling. Diverse approaches to this complex problem have been pursued on the basis of reasonable simplifications, usually appropriate to non-interacting sub-columns, and are reviewed for example by Beuermann (2004).

A fundamental description, Aizu (1973) gives with the analytical solution of a shock model, developed to describe the gas accretion in Sco X-1 and related objects. He considers a steady, radially infalling and optically thin gas flow, which is cooled by bremsstrahlung. In this concept, the shape of the X-ray spectrum depends on the stellar mass, and the luminosity is proportional to the mean accretion rate. Most of the more comprehensive models to the accretion column start from the Aizu (1973) calculations.

With the objective of effective fits to high-resolution X-ray spectra and of spectroscopic mass determinations, Cropper et al. (1999) develop further the one-dimensional hydrodynamic model of Wu et al. (1994), describing a radially symmetric, narrow accretion column. Under the assumptions of mass, momentum, and energy conservation, they include cyclotron emission perpendicular to the direction of the infalling matter (and thus to the field lines) and bremsstrahlung cooling to derive the temperature and density structure in the column. With the optically thin post-shock flow divided in stratums of constant temperature, the total spectrum can be calculated as the sum of the local spectra. Cropper et al. (1999) also take into account the dependence of the shock height on gravity effects.

Woelk \& Beuermann $(1992,1996)$ and, on the basis of their results, Fischer \& Beuermann (2001) solve the radiative transfer and the hydrodynamic equations in a stationary two-fluid plasma simultaneously, discriminating between the electron and the ion temperature distribution, which mostly differ near the shock. The radiative transfer in a plane-parallel heated flow is treated frequency-dependently and angle-dependently. By considering both bremsstrahlung and cyclotron cooling and using an artificial viscosity which connects the shock and the bombardment case, a wide range of local mass flow densities and magnetic field strengths can be covered. Fischer \& Beuermann (2001) parametrize the derived temperature profile and the geometrical shock height. The emerging X-ray spectra depend on the mass flow rate, the magnetic field strength, and the mass of the white dwarf. 


\subsubsection{Soft X-ray dominated systems}

In contradiction to the standard accretion model for MCVs described in Section 2.5.1, a number of AM Her systems show an unexpected dominance of soft over hard X-ray emission. This so-called soft X-ray excess, first stated for the case of the prototype AM Her by Rothschild et al. (1981), increases with the magnetic field strength (Beuermann \& Schwope 1994; Ramsay et al. 1994). Beuermann \& Burwitz (1995) point out that at a high magnetic field strength ( $B \gtrsim 30 \mathrm{MG}$ ) coincident with a relatively low accretion rate, cyclotron emission becomes the main cooling process, causing an enhanced soft X-ray flux. The hard X-radiation is primarily emitted from the post-shock accretion column, the extent and height of which decrease with increasing magnetic field strength (Woelk \& Beuermann 1996). Both magnetic field strength and mass flow rate thus regulate the energy balance in polars (cf. Fischer \& Beuermann 2001). Ramsay et al. (1994) include the contribution of cyclotron emission to the total luminosity from shock region and post-shock flow and use unabsorbed bolometric luminosities from ROSAT X-ray data for determining the soft-to-hard flux ratio, and still end up with a dominance of soft X-ray emission. Due to the low energy resolution and the limited energy range covered by the ROSAT PSPC detector, however, these results are uncertain and strongly model dependent. Consequently, cyclotron emission may be one, but not the only solution of the 'soft X-ray puzzle'. Widely accepted is a connection to inhomogeneous and time-dependent ('blobby') column accretion, suggested by Kuijpers \& Pringle (1982) and developed further by Frank et al. (1988). In this scenario, instabilities caused by the magnetic pressure force the stream material into inhomogeneous denser blobs, possibly near the threading point at the magnetospheric boundary. These blobs persist throughout the magnetically controlled accretion stream and can penetrate the stellar photosphere of the primary, where they are submerged and thermalized by the surrounding material, radiating away their energy in the soft X-ray and UV band (Figure 2.7). Strong fluctuations in the emitted flux are another consequence of blobby events. The phenomenon was revisited by Ramsay \& Cropper (2004) in an analysis of new XMM-Newton and re-calibrated ROSAT data. Considering geometrical effects as well as the influence of accretion 'blobs', they derive a soft-to-hard ratio close to one for the majority of the observed systems and claim that - owing to deficits in the formerly used calibration and models - fewer systems than previously thought show a notable soft $\mathrm{X}$-ray excess. This suggests that the reprocessed component in some systems is cool enough to have moved out of 


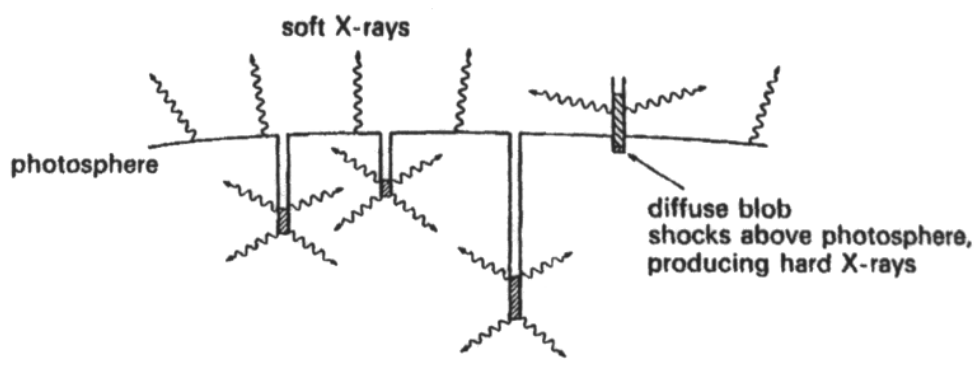

Figure 2.7: Blobby accretion scenario. Dense blobs in the accretion stream penetrate the atmosphere and bury the shock under its outer layers. From Frank et al. (2002).

the soft X-ray and into the EUV or UV band. A similar conclusion has been reached by Gänsicke (1998) based on an analysis of IUE and HST/GHRS observations. Further observational investigations on the energy balance in soft polars as performed in this work may help to clarify the picture.

On the basis of XMM-Newton and optical observations of selected soft polars, we study the physical properties of the accretion column and the accretion region in the X-ray regime. High-state data give a valuable insight into the accretion process. Topics of the analysis are the flux contributions and variations of the system components, their spectral signature and temperature structure, examined by comparing different spectral models, and the long-term variability. The work concentrates on two systems with diverse physical parameters periods, magnetic fields, temperatures, and accretion rates - which both have shown a soft X-ray excess in former ROSAT observations. Adding these to the sample of objects that have been studied on the basis of high-resolution X-ray data, we aim to test the controversial propositions on the energy balance of soft polars. 


\section{CHAPTER 3}

\section{Modeling the X-ray spectra of MCVs}

\subsection{Spectral fitting by means of XSPEC}

In order to deduce the physical parameters of an observed astrophysical object from its spectrum, a model is required that reproduces both the continuum and the spectral lines. In the infrared, optical, and ultraviolet wavelength ranges, the main conclusions can be drawn from the shape and the depth of the spectral lines. The knowledge of the atomic physical conditions and the element transitions in the X-ray regime is still deficient, as the corresponding high temperatures and pressures can hardly be studied under laboratory conditions. Moreover, in most cases the energy resolution of current X-ray spectra is too low to apply line diagnostics and similar techniques, so alternatives are needed. The continuum shape of an X-ray spectrum gives important clues on the underlying physical processes (Section 2.5). Thus, a realistic spectral model has to consider the generation of the continuum, line formation and blending effects, and the dependence of the observed spectra on the detector efficiency. The correlation between the actual, continuous source spectrum $f(E)$ against the energy $E$ and the discrete observed spectrum $C_{\text {source }}(I)$ in counts per detector channel $I$ can be described as

$$
C_{\text {source }}(I)=\int_{0}^{\infty} f(E) R(I, E) A(E) d E
$$

The detector response comprises the redistribution matrix $R(I, E)$, which gives the detection probability per input X-ray energy $E$ and observed spectrometer channel $I$, and the effective area $A(E)$. To derive the actual source spectrum 
from the observed one, Equation 3.1 has to be inverted. This is not readily possible, and a forward approach is usually chosen that determines a model version of $f(E)$ by a least-squares fit. The standard procedure applied by the fitting scheme of the interactive X-ray spectral-fitting program XSPEC (Dorman \& Arnaud 2001; Dorman et al. 2003; Arnaud 1996, and references therein) starts with the calculation of a theoretical model for an estimated parameter set. The predicted model spectrum $C_{\text {model }}(I)$ in counts per channel $I$ is convolved with the instrumental response and compared to the observed data, by default using a modified Levenberg-Marquardt algorithm for non-linear least-squares problems (Levenberg 1944; Marquardt 1963; Bevington \& Robinson 2003) and $\chi^{2}$ statistics. The theoretical model is varied by changing the relevant parameters until the goodness-of-fit criteria are fulfilled. The quantity

$$
\chi^{2}=\sum \frac{\left(C_{\text {source }}(I)-C_{\text {model }}(I)\right)^{2}}{(\sigma(I))^{2}},
$$

where $\sigma(I)$ is the error per channel, gives a measure for the fit quality. A reasonable fit will result in a reduced $\chi_{\text {red }}^{2}=\chi^{2} /$ d.o.f. close to one, with $\chi_{\text {red }}^{2}$ being defined as $\chi^{2}$ divided by the number of degrees of freedom. A confidence interval for an individual parameter is achieved by varying the parameter within a (specified) range around the best-fit value. For complex data and models, several acceptable fits may pass the good-fit conditions, obliging the user to control the fit reliability.

The spectral analysis presented in this work is based upon XSPEC v12.212.5 (Arnaud et al. 2008). The package has been developed with the objectives of handling multiple datasets interactively, instrument-independently, and simultaneously. It manipulates X-ray source and background data, calibration data, and built-in theoretical models with the option to implement additional models. 'Additive' model components represent different X-ray sources or radiation processes, while 'multiplicative' components describe modifications by other physical processes like interstellar or intrinsic absorption. Further features are the simulation of spectra for given models and detector responses, and a simultaneous multi-source fitting for coded-mask instruments.

A set of different XSPEC models has been applied in order to describe the different radiation processes in magnetic cataclysmic variables (Section 2.5) and shall be summarized in the following. 

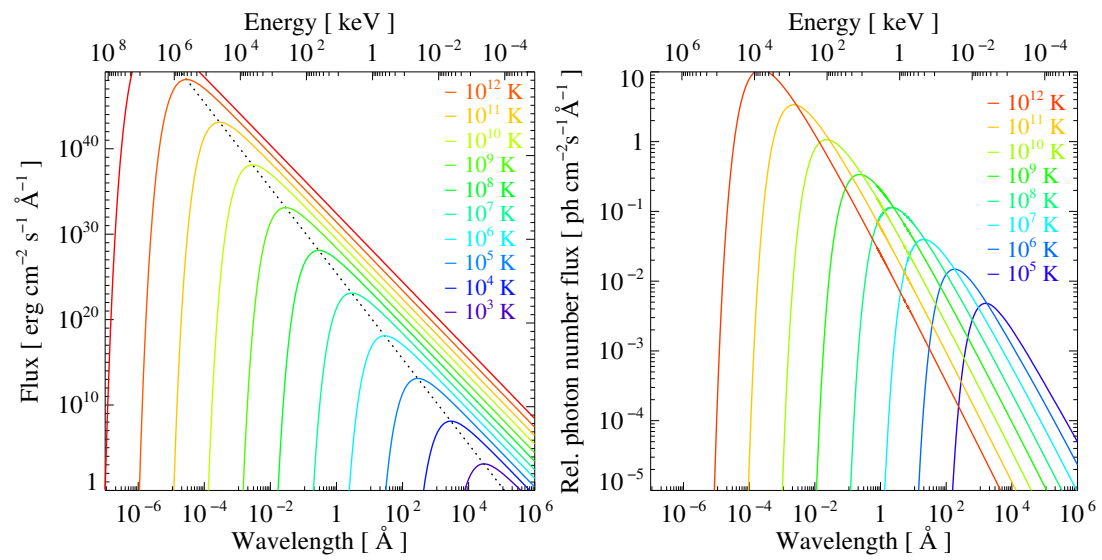

Figure 3.1: Two main radiation processes in MCVs. Left: Planck functions for increasing temperatures of the emitting object and Wien's Displacement Law (dotted line). Right: Thermal bremsstrahlung at different emission temperatures.

\subsubsection{Black body radiation}

The black body concept assumes an object in thermal equilibrium. Max Planck (1858 - 1947) described the spectral distribution of black body radiation postulating the quantization of energy in the interaction between light and matter, and derived the empirical formula for the intensity

$$
B_{\lambda}=\frac{8 \pi h c}{\lambda^{5}} \cdot \frac{1}{e^{h c / \lambda k T}-1}
$$

where $h$ is Planck's constant, $c$ the speed of light in vacuum, and $k$ the Boltzmann constant. This continuum spectral energy distribution is characteristic for each black body temperature $T$ (left panel in Figure 3.1). The wavelength at which the emission reaches maximum intensity is given by Wien's Displacement Law

$$
\lambda_{\max }=\frac{b}{T}
$$

with Wien's constant $b$, again depending only on the temperature.

The Planck formula (Equation 3.3) is implemented in the form of two equivalent XSPEC models, which primarily differ in the physical meaning of their 
normalizations. BBODY obeys

$$
f_{\mathrm{BB}}(E)=K_{\mathrm{BB}} \cdot \frac{8.0525 E^{2} d E}{(k T)^{4}\left(e^{E / k T}-1\right)}
$$

with the norm $K_{\mathrm{BB}}=L_{39} / D_{10}^{2} . \quad L_{39}$ is the source luminosity in units of $10^{39} \mathrm{erg} \mathrm{s}^{-1}$ and $D_{10}$ the distance to the source in units of $10 \mathrm{kpc}$. The normalization $K_{\mathrm{BBR}}=R^{2} / D_{10}^{2}$ of BBODYRAD represents the emitting surface area in terms of the source radius $R$ in $\mathrm{km}$ and the distance to the source. The model flux is given by

$$
f_{\mathrm{BBR}}(E)=K_{\mathrm{BBR}} \cdot \frac{1.0344 \cdot 10^{-3} E^{2} d E}{e^{E / k T}-1}
$$

\subsubsection{Thermal plasma emission}

According to classical electrodynamics, accelerated (or decelerated) charged particles irradiate, mainly perpendicularly to the direction of the acceleration. Traversing the surrounding matter, the free electrons interact with the Coulomb field of the shell electrons and the nuclear charge of the atoms. The emerging radiation is called bremsstrahlung or free-free emission. High-energy electrons in astrophysical plasmas are braked by excitation and ionization of the atoms. The radiation energy depends on the velocity of the electron and hence on the temperature of the environment. In thermal equilibrium, the electrons follow a Maxwellian velocity distribution and emit a characteristic continuum spectrum in dependence on the temperature (right panel in Figure 3.1). This thermal bremsstrahlung spectrum can be described as

$$
I(E, T) \propto G_{\mathrm{ff}}(E, T) Z^{2} n_{\mathrm{e}} n_{\mathrm{i}} \frac{e^{-E / k T}}{\sqrt{k T}},
$$

where $Z$ is the ion charge number, $n_{\mathrm{e}}$ the electron and $n_{\mathrm{i}}$ the ion density (Charles \& Seward 1995). The Gaunt factors $G_{\mathrm{ff}}$, slowly increasing as the energy $E$ decreases, have been calculated for example by Karzas \& Latter (1961). The XSPEC model BREMSS adapts the method of Kellogg et al. (1975), who interpolate the Karzas \& Latter (1961) Gaunt factors by second-order polynomial fits. The only free parameter of the model is the plasma temperature $k T$ (Figure 3.1). 
The real spectrum of a hot plasma shows line formation in addition to the thermal bremsstrahlung-like continuum. MEKAL plasma emission models (Mewe et al. 1985, 1986; Liedahl et al. 1995) include the contribution of the continuum and of the element lines from a hot, optically thin plasma under the assumptions of collisional ionization equilibrium and Maxwellian electron and ion energy distributions. The calculation of the continuum radiation involves free-free, free-bound, and two-photon emission. The line transitions stem from electron impact, radiative and dielectronic recombination, and from inner-shell excitation and ionization. The XSPEC MEKAL model adopts the ionization balance from Arnaud \& Rothenflug (1985) and Arnaud \& Raymond (1992) and has a normalization corresponding to the emission measure. The solar metal abundance ratios used in this work follow the results of $3 \mathrm{~d}$ hydrodynamic calculations by Grevesse et al. (2007), increasing the mean metal abundance by about $20 \%$ compared to the values of Anders \& Grevesse (1989).

\subsubsection{Cooling flow models}

Cooling flow models describe the energy loss by radiative cooling in an optically thin plasma at low densities. Mushotzky \& Szymkowiak (1988) introduce a model for cooling flows in galaxy clusters, which sums the contributions of plasma photon spectra over a distribution of temperatures or emissivities respectively. The mass flow rate is assumed to be constant throughout the cooling flow. The observed spectrum

$$
\operatorname{Obs}(E) \propto \frac{\dot{M}}{4 \pi D^{2}} \int \frac{P(E, T)}{\varepsilon_{\text {total }}(T)} d T
$$

depends on the plasma photon spectrum $P(E, T)$ at a temperature $T$ in the energy bin $E$, on the mass flow rate $\dot{M}$, and on the total bolometric emissivity $\varepsilon_{\text {total }}(T)$ from the shell at $T$. As in most plasma models, the element abundance strongly correlates with the other parameters, in particular with temperature, density, and mass flow rate. The CFLOW and MKCFLOW models in XSPEC, relying on Mushotzky \& Szymkowiak (1988), are the sum of ten MEKAL emission models with the total normalization representing the mass flow rate. CFLOW includes a power law distribution of the emissivity versus temperature, while in the MKCFLOW model, the emissivity stays constant for shells having temperature intervals of the same size. 


\subsubsection{Galactic and intrinsic absorption}

XSPEC offers several models to account for photo-electric absorption in the interstellar medium. PHABS makes use of the cross sections from BalucinskaChurch \& McCammon (1992) and Yan et al. (1998) and follows

$$
f(E)=e^{-N_{\mathrm{H}} \sigma(E)},
$$

where $N_{\mathrm{H}}$ represents the equivalent hydrogen column and $\sigma(E)$ the photoelectric cross-sections without Thomson scattering. A more complex approach is pursued in the TBABS package (Wilms et al. 2000), which incorporates the galactic absorption due to gas, grains, and $\mathrm{H}_{2}$ molecules. The cross sections stem from Verner \& Ferland (1996) and Verner et al. (1996). The most recent version $\mathrm{TBNEW}^{1}$, used in this work, refines the cross-section calculation around the iron, oxygen, and neon edges.

In order to reproduce intrinsic absorption by partially ionized material in the systems, warm absorber models can be chosen. ABSORI (Done et al. 1992; Zdziarski et al. 1995) also considers the spectral impact of Compton reflection, resorting to photoionization rates from Reilman \& Manson (1979) and to recombination rates from Shull \& van Steenberg (1982). The model depends on the temperature of the absorbing material, its ionization degree, and the spectral shape which is approximated by a power law. Cross sections at energies above $5 \mathrm{keV}$ are extrapolated. The XSTAR package (Kallman \& Bautista 2001; García et al. 2005) provides warm absorber and emitter spectra from photoionized material with the parameters element abundances, ionization, degree of broadening by turbulence, and - for the absorber model - column density.

An alternative which accounts for the geometric properties of the system are partial covering absorbers. The PCFABS model is closely related to the PHABS photo-electric absorption and introduces the dimensionless covering fraction factor $x$ between 0 and 1 :

$$
f(E)=x e^{-N_{\mathrm{H}} \sigma(E)}+(1-x),
$$

which is in particular convenient for the phase-resolved spectroscopic analysis of magnetic cataclysmic variables. PARTCOV allows using any absorber model as partially covered.

\footnotetext{
1 http://pulsar.sternwarte.uni-erlangen. de/wilms/research/tbabs/ provides further explanations.
} 


\subsection{A multi-temperature black body approach to the accre- tion region}

The standard approach to model the spectral signature of the accretion region in MCVs is a single absorbed black body, representing a mean temperature of the hot white-dwarf atmosphere throughout the spot. The real temperature structure of the region, which is heated by irradiation and by dissipation of kinetic energy, is still widely unknown. It has to cover a wide range of temperatures between the base of the column and the unheated surface of the star. The empirical multi-temperature black body model approximates the effective surface temperature of the accretion region by a Gaussian temperature distribution. The highest temperature $k T_{\max }$ is found at the center of the spot, while the temperature limit for large distances from the center is the effective temperature $k T_{*}$ of the white dwarf. The Gauss function is discretized by approximating the spot with concentric rings of constant width initially. To each ring, the temperature at its mean distance from the region center is assigned. In order to end up with temperature steps not exceeding $5 \mathrm{eV}$ between neighbored components for the typical parameter range in polars, we opt for a maximum number of fifteen rings. The outer boundary of the accretion region is defined at that distance, where the temperature reaches $1 \%$ of the difference between $k T_{\max }$ and $k T_{*}$. The temperature distribution in dependence on the ring number $n$ is then given by

$$
k T_{\mathrm{n}}=k T_{*}+\left(k T_{\max }-k T_{*}\right) \cdot e^{\ln 0.01 \cdot n(n-1) / 15 \cdot 14},
$$

and the associated emitting surface areas are

$$
A_{\mathrm{n}}=(2 n-1) A\left(k T_{\max }\right) \text {. }
$$

A derivation of these relations can be found in Appendix A. On their basis, the Gaussian-temperature model has been implemented in XSPEC as an additional model, following the XSPEC model BBODYRAD, the normalization of which is proportional to the emitting surface area (Equation 3.6), and summing up the black body components. Equation 3.11 describes the temperature of each model component in dependence on $k T_{*}$ and $k T_{\max }$, while Equation 3.12 couples the effective areas via the BBODYRAD norm to the one of the hottest component. The resulting spectrum shows a shallower slope than a singletemperature black body (Figure 3.2). The highest X-ray flux arises from the hottest components with small effective areas, while the low-temperature black bodies covering larger surface areas contribute only little to the total soft X-ray 


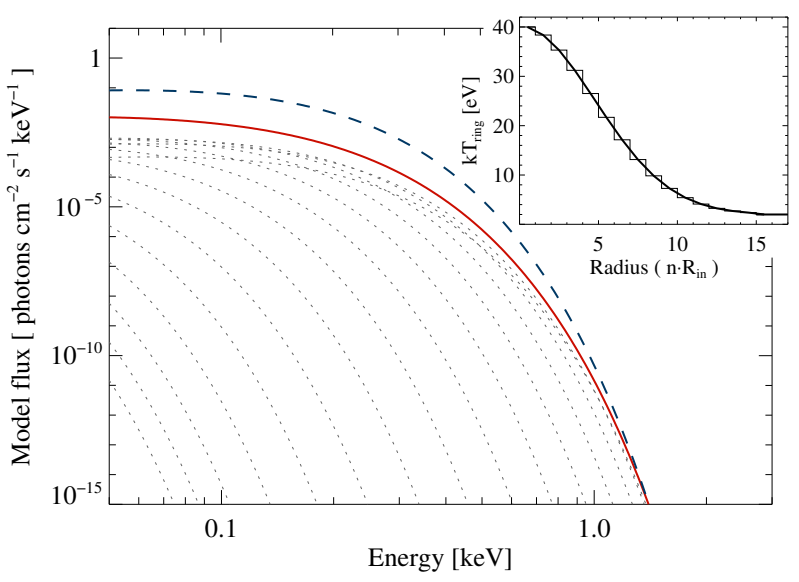

Figure 3.2: A 15-temperature black body model (red) with its individual components between 2 and $40 \mathrm{eV}$ (dotted) compared to a single black body at $35 \mathrm{eV}$ (dashed) with the X-ray flux and the emitting surface area corresponding to the multi black body. The small panel shows the discretized Gaussian temperature distribution.

Table 3.1: Temperatures, normalizations, and contributions to the flux in the XMMNewton energy range $0.1-10.0 \mathrm{keV}$ and to the bolometric flux for an example multi black body model with $k T_{\max }=40 \mathrm{eV}$ and $k T_{*}=2 \mathrm{eV}$.

\begin{tabular}{rrrcc}
\hline \hline$n$ & $\mathrm{kT}[\mathrm{keV}]$ & norm & $F_{\text {n }} / F_{\text {XMM }}$ & $F_{\text {n }} / F_{\text {bol }}$ \\
\hline 1 & 40.0 & 0.004 & $1.02 \mathrm{e}-01$ & $7.96 \mathrm{e}-02$ \\
2 & 38.4 & 0.013 & $2.51 \mathrm{e}-01$ & $2.03 \mathrm{e}-01$ \\
3 & 35.3 & 0.022 & $2.78 \mathrm{e}-01$ & $2.41 \mathrm{e}-01$ \\
4 & 31.2 & 0.031 & $2.07 \mathrm{e}-01$ & $2.06 \mathrm{e}-01$ \\
5 & 26.5 & 0.040 & $1.10 \mathrm{e}-01$ & $1.38 \mathrm{e}-01$ \\
6 & 21.7 & 0.049 & $4.08 \mathrm{e}-02$ & $7.58 \mathrm{e}-02$ \\
7 & 17.1 & 0.058 & $9.45 \mathrm{e}-03$ & $3.46 \mathrm{e}-02$ \\
8 & 13.1 & 0.066 & $1.23 \mathrm{e}-03$ & $1.37 \mathrm{e}-02$ \\
9 & 9.8 & 0.075 & $7.17 \mathrm{e}-05$ & $4.86 \mathrm{e}-03$ \\
10 & 7.3 & 0.084 & $1.67 \mathrm{e}-06$ & $1.68 \mathrm{e}-03$ \\
11 & 5.4 & 0.093 & $1.04 \mathrm{e}-08$ & $5.55 \mathrm{e}-04$ \\
12 & 4.1 & 0.106 & $2.44 \mathrm{e}-11$ & $2.11 \mathrm{e}-04$ \\
13 & 3.2 & 0.111 & $2.02 \mathrm{e}-14$ & $8.15 \mathrm{e}-05$ \\
14 & 2.7 & 0.119 & $5.57 \mathrm{e}-17$ & $4.46 \mathrm{e}-05$ \\
15 & 2.4 & 0.128 & $5.14 \mathrm{e}-19$ & $2.99 \mathrm{e}-05$ \\
\hline
\end{tabular}


emission. Increasing the surface temperature from $1 \mathrm{eV}(11600 \mathrm{~K})$ to its tenfold $10 \mathrm{eV}$ at a maximum temperature of $40 \mathrm{eV}$, for example, changes the total flux in the XMM-Newton energy range only by $5 \%$. Thus, in most cases the temperature and the surface area of the coolest component can remain fixed, and the only free parameters of the model are the temperature and the normalization of the hottest black body. Table 3.1 lists the temperatures, normalizations (proportional to the emitting surface areas), and flux contributions for an example model with $k T_{\max }=40 \mathrm{eV}$ and $k T_{*}=2 \mathrm{eV}$.

Developed on the basis of a rigorously simplified picture of the accretion region with the objective of minimizing the complexity of the equations, this model eventually accounts for a more realistic und thus more complex geometry in which the emitting surface areas associated to the different temperatures obey a similar distribution: small regions of high temperatures, extended regions of lower temperatures. Further comparisons between a singletemperature black body and the multi-component black body models can be found in the spectral analyses of the magnetic cataclysmic variables AI Tri and QS Tel in Sections 5.3 and 6.4.

\subsection{A multi-temperature plasma approach to the accretion column}

The post-shock accretion flow, where the plasma is decelerated and cooled, holds a complex structure (see Section 2.5). The main cooling processes arise from bremsstrahlung and cyclotron radiation. Their efficiency - and thus the density and temperature distribution in the post-shock flow - depends on the local mass flow density, on the magnetic field strength, and on the mass of the primary. Our multi-temperature plasma approach to model the associated hard $\mathrm{X}$-ray spectrum bases on the emission region models of Fischer \& Beuermann (2001). They calculate the radiative transfer for bremsstrahlung and cyclotron radiation in a stationary two-fluid plasma and establish temperatures and column densities for different local mass flow rates $\dot{m}$ and magnetic field strengths $B$ (cf. Section 2.5.2). Figure 3.3 shows the temperature distribution in the postshock flow as a function of the distance to the shock front for various sets of $B$ and $\dot{m}$ according to Fischer \& Beuermann (2001). We adopt their temperature and density structures to account for the wide range of temperatures in the post-shock accretion column and to derive relations between the temperatures and the emission measures of several optically thin MEKAL components. 


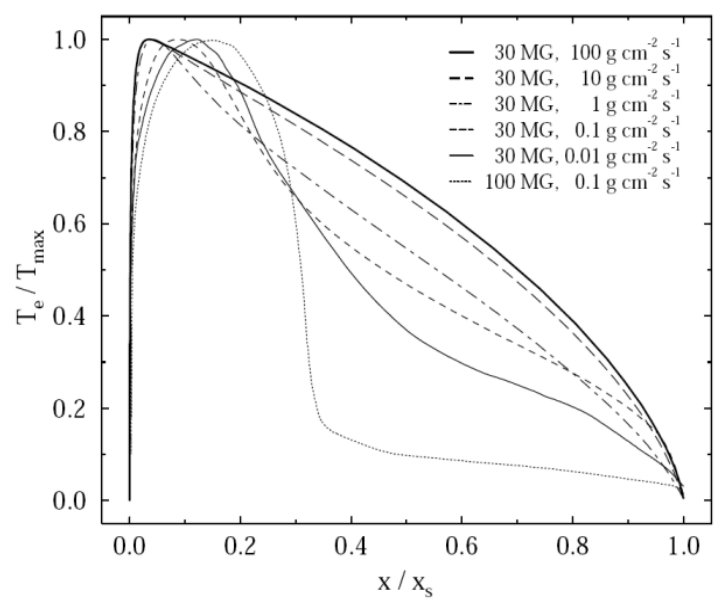

Figure 3.3: Normalized temperature structure in the post-shock flow. The electron temperature is given as a function of the column density for different sets of magnetic field strength and local mass flow density. The column density $x$, a measure for the distance to the shock front, equals $x_{\mathrm{s}}$ at the white dwarf surface and zero at the shock. From Fischer \& Beuermann (2001).

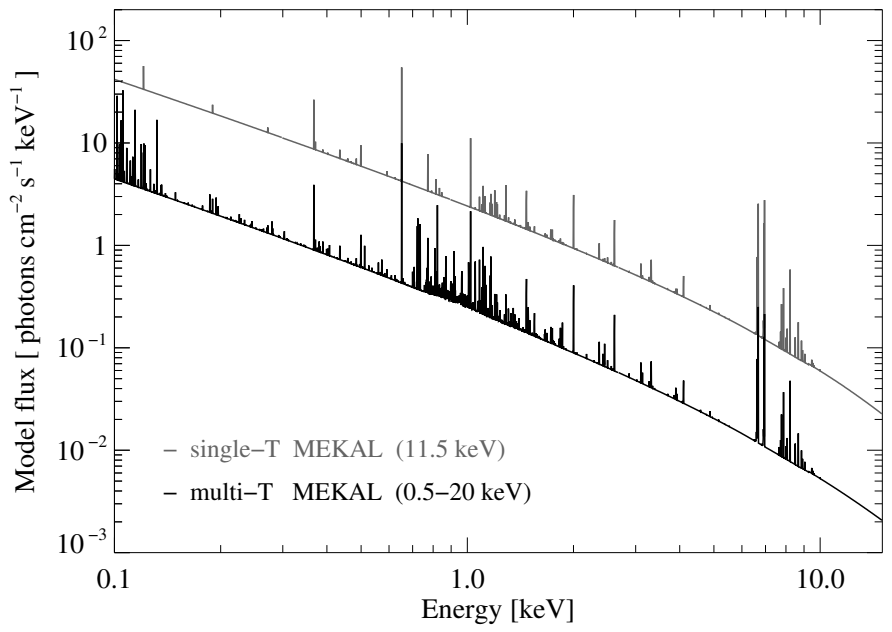

Figure 3.4: Single- (grey) and multi-temperature (black) MEKAL models. The single MEKAL spectrum is shifted by a factor of 10 for clarity. 
Under the assumption that optically thin conditions dominate, the X-ray emission of the accretion column is then approximated by the sum of seventeen MEKAL spectra. The parameter range covers local mass flow densities between $\dot{m}=0.01$ and $100 \mathrm{~g} \mathrm{~cm}^{-2} \mathrm{~s}^{-1}$ and magnetic field strengths of $B=10,30$, and $100 \mathrm{MG}$ for a characteristic primary mass of $\mathrm{M}_{\mathrm{WD}}=0.6 \mathrm{M}_{\odot}$. The temperature structures for other values of $B$ and $\dot{m}$ are estimated by means of a bilinear interpolation over the grid of Fischer \& Beuermann (2001). The highest mass flow densities apply to the case of dense blobs in the accretion stream (Beuermann 2004). The element abundance, the temperature of the coolest component, and the emission measure are the free parameters for each $B-\dot{m}$ combination. Additionally, we assume the intrinsic absorption of the individual model components as uniform enough to be described by a single common absorption term assigned to the total spectrum. An example column spectrum with temperatures between $20.0 \mathrm{keV}$ and $0.5 \mathrm{keV}$ is displayed in Figure 3.4, compared to a single MEKAL model at $k T_{\mathrm{MEKAL}}=11.5 \mathrm{keV}$, which corresponds to the flux weighted mean of the individual constituents of the composite spectrum. The cooler components strengthen the emission lines at energies below $3 \mathrm{keV}$, while the hotter components raise the continuum above $2 \mathrm{keV}$. Both the single- and the multi-temperature MEKAL models are employed in the spectral analysis in the course of this work.

\subsection{Caveats and conclusions}

The physical properties of the accretion column and the accretion region have been subject to diverse theoretical approaches and observational studies. Our multi-temperature models work as an approximation to the temperature distributions, but do not claim to provide an accurate physical description of the accretion process. At present, all the theoretical models used in spectral analyses of MCVs rely on various assumptions to simplify the complex situation (Section 2.5.2). Their parameters are closely connected and directly respond to small changes in the model. The element abundance for example is highly dependent on the temperature structure and the accretion rate. Therefore, one should be very cautious in deriving system parameters like the white-dwarf mass, the magnetic field, or the accretion rate from such spectral fits - in fact, they are rather prerequisites than results. The spectral resolution of the currently available X-ray data of magnetic cataclysmic variables also limits the determination of the parameters and the evaluation of the different methods to 
model the emission regions. Considering the temperature and density distribution, nonetheless, can improve the fit quality and provides a more realistic description than the single-temperature approaches. The Fischer \& Beuermann (2001) models to the post-shock flow stand for sub-columns of identical mass flow densities. A comprehensive model would consist of various sub-columns over several magnitudes of $\dot{m}$, each with a separate absorption term, as outlined for instance by Rousseau et al. (1996) and Beuermann (2004). Again limited by the spectral resolution, the deduction of a distribution in local mass flow densities, however, is currently beyond the means of an analytical study. 


\section{CHAPTER 4}

\section{$\mathrm{X}$-ray instrumentation}

\subsection{Missions overview}

When the X-ray activity of the Sun was measured for the first time by a photon counter on-board a sounding rocket in the 1940s (Friedman et al. 1951), its low intensity led to the presumption that it should not be possible to detect any X-ray source outside the solar system. In 1962 however, the field of $\mathrm{X}$-ray astrophysics commenced with another rocket-borne experiment. During its flight, lasting only a few minutes, the diffuse cosmic X-ray background and the first discrete celestial X-ray source Sco X-1 were discovered (Giacconi et al. 1962). A rapid development of the cosmic X-ray research started, involving technological advances and increasing instrumental sensitivity. The early instruments comprised proportional and scintillation counters as known from particle physics, providing a low spatial and energy resolution. Additional mechanical collimators reduced the field of view and introduced the option of indirect imaging by successively scanning larger regions of the sky. After the sounding rockets carrying Geiger photon counters, balloons and crystal scintillators opened the window towards higher energies between 20 and $60 \mathrm{keV}$. From 1970 to 1973, UHURU, the first satellite exclusively dedicated to cosmic $\mathrm{X}$-ray astrophysics, collected an X-ray sky map and revealed more than 300 $\mathrm{X}$-ray sources.

Riccardo Giacconi and Bruno Rossi in 1960 adopted an idea developed by Hans Wolter almost twenty years ago for the construction of microscopes (Giacconi 2003): Reflection of X-rays becomes possible under small angles, establishing focusing capabilities. The so-called Wolter type I mirror consists of two coaxially mounted parts, the front one of parabolic and the back one of hyperbolic shape (Figure 4.1), which double reflect the incident light into a 

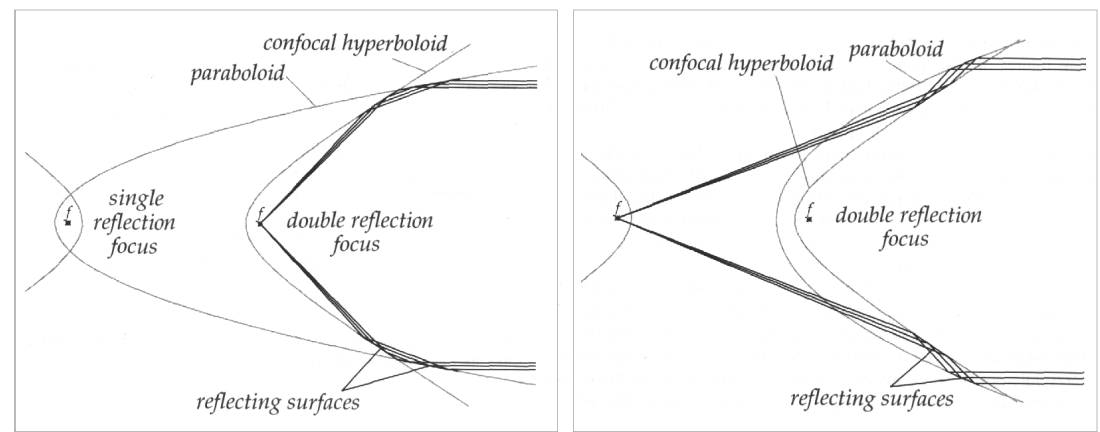

Figure 4.1: Schematic view of the Wolter telescopes type I (left) and II (right). Adapted from Friedrich (2008).

common focus. In 1979, for the first time a satellite was equipped with a Wolter I telescope: HEAO-2, later-on renamed to Einstein. Present-day instruments like XMM-Newton carry multiple nested systems of Wolter-type mirrors.

A multiplicity of dedicated and non-dedicated missions followed UHURU, which have essentially extended the catalog of known objects and classes. Among the most prominent were the X-ray and $\gamma$-ray HEAO series in the 1970, the European EXOSAT and the Japanese GINGA in the 1970, the Roentgen Satellite ROSAT (1990-1999), which combined a large all-sky survey with a variety of pointed observations, the still working Rossi X-ray Timing Explorer RXTE, started in 1995, the NASA mission Chandra and the ESA satellite XMM-Newton (both launched in 1999), which feature a considerable spectral and spatial resolving power, or the ESA $\gamma$-ray observatory INTEGRAL (since 2002). CCDs considerably improved the energy resolution and widened the energy range supplied. The X-ray targets comprise for instance neutron stars, active galactic nuclei, quasi-stellar objects, or pulsars. High-resolution grating spectrographs, as currently employed in XMM-Newton and Chandra and as planned for future missions, allow for detailed spectroscopic studies of X-ray relevant astrophysical processes.

The following Sections are dedicated to the instrumentation of the XMMNewton and ROSAT satellites which this work is based onto. 


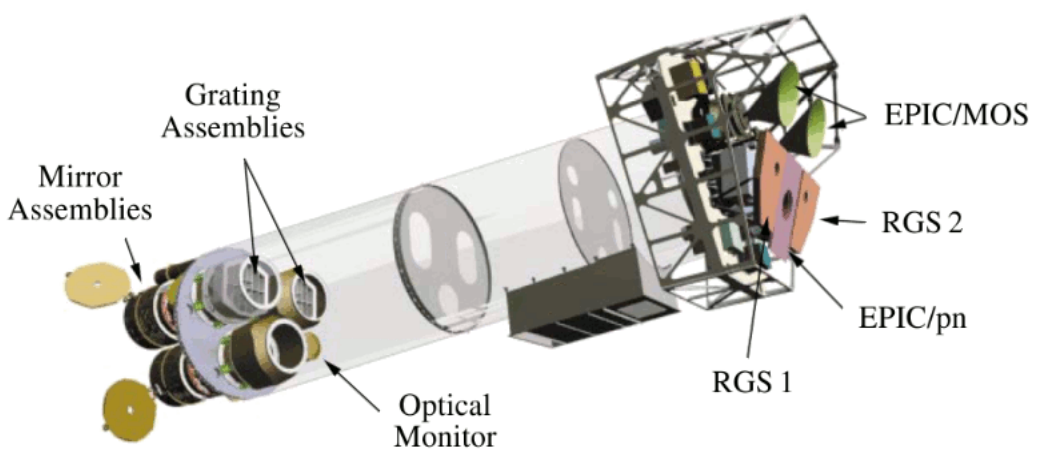

Figure 4.2: Sketch of the XMM-Newton payload with the different X-ray instruments, showing the three Wolter I mirror assemblies at the lower left, and at the right end the instrument platform with EPIC/MOS, EPIC/pn, and RGS. The OM camera in the lower part of the payload hides behind the mirror modules. Adapted from Jansen et al. (2001).

\subsubsection{XMM-Newton}

The ESA X-ray Multi Mirror Mission XMM-Newton (Jansen et al. 2001; Ehle et al. 2007), honoring Sir Isaac Newton (1643 - 1727), has been launched in December 1999 into a highly eccentric 48 hours orbit. Its primary objective has been high quality spectroscopy over a large energy range. The three parallel mounted telescopes (Figure 4.2) with a large effective area comprise 58-fold nested Wolter type I mirror assemblies and reach an angular resolution of 15 arcsec half energy width at $1.5 \mathrm{keV}$ and $8 \mathrm{keV}$. Six instruments work largely independently and allow for simultaneous data acquisition in different wave bands and observation modes (cf. Figure 4.3).

The three focal plane detectors of the European Photon Imaging Camera (EPIC) system with circular fields of view of 30 arcmin in diameter (Turner et al. 2001) are located behind the three telescope mirrors. They obtain spectra at an energy resolution of $E / \Delta E=20-50$ and high quantum efficiency in the energy range between $0.1 \mathrm{keV}$ and $10.0 \mathrm{keV}$, covering the main $\mathrm{K}$ shell transitions of light and $\mathrm{L}$ shell transitions of heavy elements. The EPIC instruments are equipped with identical filter wheels with four optical and UV blocking filters of different thickness each - two thin, one medium, one thick -, designated 


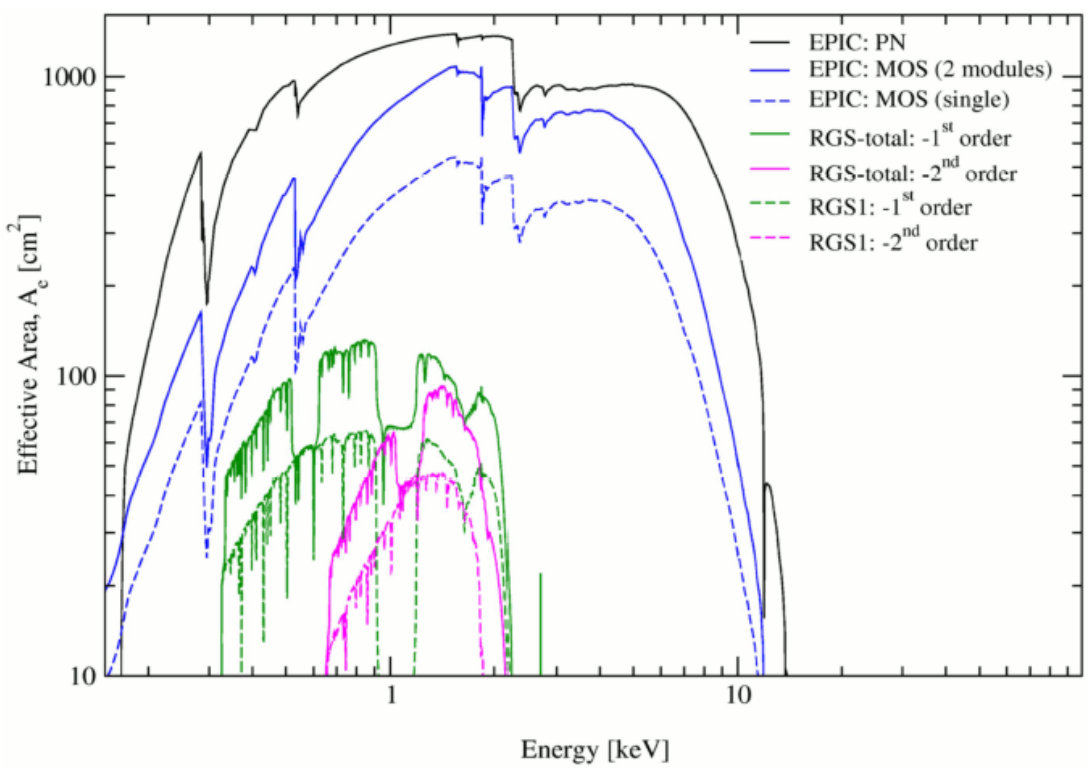

Figure 4.3: Net effective area and energy bands covered by the X-ray instruments EPIC and RGS on-board XMM-Newton. From Ehle et al. (2007).

to targets of different brightness and spatial extent. The EPIC/pn CCD camera (Strüder et al. 2001), based on a p-n technology and the concept of sideward depletion, consists of twelve individually operated back-illuminated CCDs on a single wafer. It provides a high time resolution of up to $7 \mu$ s (Ehle et al. 2007), but with $1.1 \operatorname{arcsec} \mathrm{px}^{-1}$ a lower angular resolution than EPIC/MOS. The two Metal Oxide Semi-Conductor (MOS) cameras (Strüder et al. 2001) share the light paths with the Reflection Grating Spectrometers (Figure 4.4). Each holds seven (MOS1: six, since one CCD failed in March 2006) front-illuminated CCDs in the focal plane with one read-out node for the outer six (five) and one for the innermost CCD and reaches a spatial resolution of $1.1 \operatorname{arcsec} \mathrm{px}^{-1}$. The EPIC cameras are operated in photon counting mode with an on-board pre-processing of the data, providing so-called event lists, which comprehend - in table form - information per received event like position, arrival time, and energy. These event lists, assuring effective data compression and thus saving telemetry, are the basis for the further data extraction and for an imaging, 


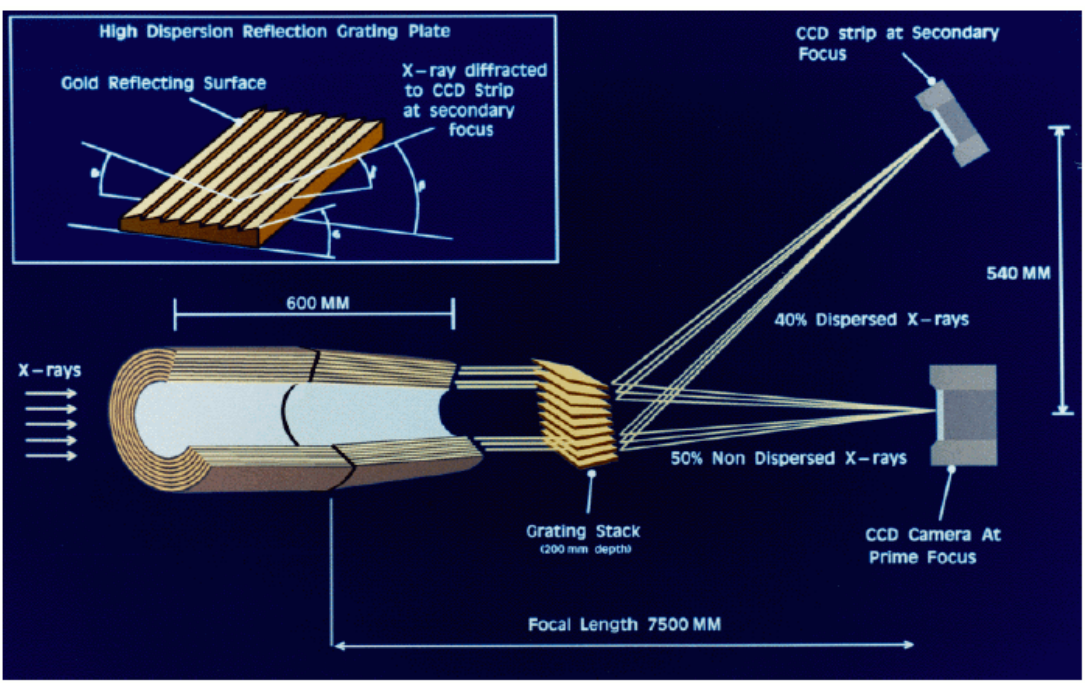

Figure 4.4: Light path in two of the three XMM-Newton telescopes (illustration not to scale). Grating assemblies deflect about $40 \%$ of the incident light to the RGS detector in the secondary focus, about $45 \%$ reach the MOS camera in the prime focus directly. From Ehle et al. (2007).

photometric, and spectroscopic analysis. Two different operational modes are available: the imaging mode with different frame configurations for spatially resolved event lists; the timing mode (comprising the burst mode) for an increased time resolution waiving the two-dimensional position information. The EPIC data of bright sources are occasionally affected by pile-up effects, when two or more photons reach the same pixel or a sample of closely neighbored pixels within one read-out cycle. The seemingly higher amplitude assigned to such an event results in an overestimation of its energy and thus in delusive hardening of the spectrum. On the other hand, the number of counts in the affected pixel(s) are underestimated, dimming the resulting image. Whilst in the case of the EPIC/MOS detector pile-up causes a loss of flux, but only little distortion of the energy spectrum, the effects on EPIC/pn spectra are evident and have to be considered in a thorough analysis. The pile-up probability calculates from the probability function of statistical (Poissonian) distributed events: 


$$
P_{\text {pile-up }}=1-e^{-C R \cdot t_{\text {readout }}}
$$

with the count rate $C R$ and the readout time $t_{\text {readout }}$ gives the chance that two events reach the detector within one readout cycle. Related phenomena with contrary effects are the optical and the X-ray loading of observations. Photons from an optically or UV bright source - either one close to the target or even the target itself - may pass the blocking filters, increasing the noise and changing the detector charges. Optical loading evidently affects thin filter exposures the most and results in an energy scale shift of $3.65 \mathrm{eV}$ per optical photon (Smith 2008). To compensate for it and to avoid spectral hardening, the current energy shifts are included in the offset map, which is calculated for each exposure. In the case of sources with high X-ray flux, X-ray events can erroneously contribute to the energy shift in the offset map, softening the source spectra (Smith 2004).

The Optical Monitor (OM, Mason et al. 2001) in Ritchey Chrétien design works simultaneously with the X-ray telescopes in the optical and ultraviolet wavelength range between $1700 \AA$ and $6500 \AA$ and is equipped with an assembly of broadband color filters. Mounted parallel to the X-ray mirror modules, it encompasses a 17 arcmin field of view central within the X-ray FOV. Its performance is optimized for the detection of faint sources up to about $24 \mathrm{mag}$. The imaging mode for highly resolved images and the fast mode for additional timing information at the expense of spatial coverage can be combined using five user defined frames. Exposure times range between $0.8 \mathrm{ksec}$ and $5.0 \mathrm{ksec}$ with an overhead of $0.3 \mathrm{ksec}$ per exposure. Two grisms for low-resolution spectroscopy and an image expander for enhanced spatial resolution are available.

Two identical Reflection Grating Spectrometers (RGS, den Herder et al. 2001) are mounted behind two of the three Wolter I mirrors and share their optical paths with the EPIC/MOS detectors (Figure 4.4). An array of 181 grazing-incidence reflection gratings in each RGS diffracts the light onto eight (originally nine) back-illuminated CCDs, operated in frame transfer mode. The RGS spectra span the soft X-ray range between $0.33 \mathrm{keV}$ and $2.5 \mathrm{keV}$ with an energy resolution of $E / \Delta E=100-500$ and a wavelength accuracy of $7 \mathrm{~m} \AA \mathrm{rms}$. The relatively small effective area limits the RGS observations to bright objects; most cataclysmic variables for example are too faint to collect a fair number of counts with RGS. 

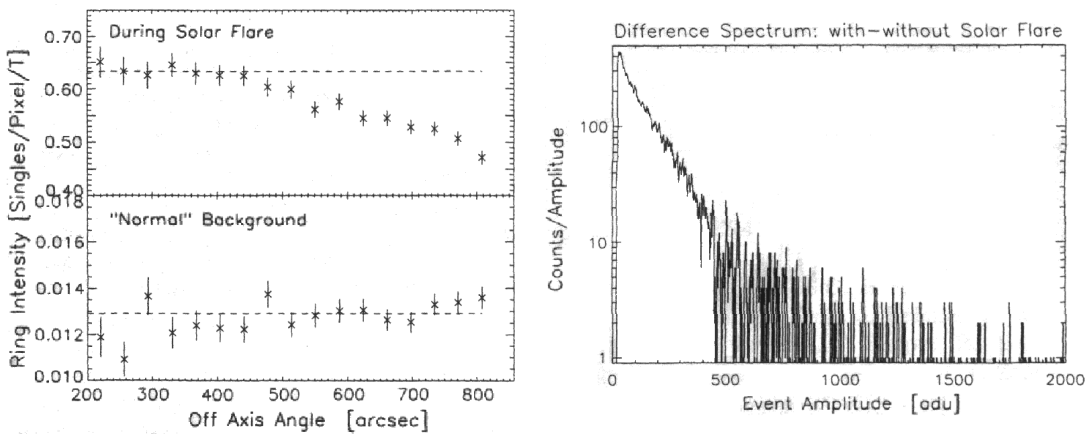

Figure 4.5: Effects of soft proton flares. Left: Encircled ring intensity over off-axis angle for the flaring and the quiescent background. Right: Difference between the flaring and the quiescent background spectra. Adapted from Strüder et al. (2000).

\subsubsection{The influence of an enhanced $X$-ray background}

In-orbit performance tests of the EPIC cameras evinced that the detected background signal was decidedly higher than reckoned in the ground calibration, mainly due to an underestimate of the particle flux in space by more than a factor of two (Briel et al. 2000) and to the large effective collecting area of the XMM-Newton mirrors. The contributions and calibration treatment of the four main background components - minimum ionizing particles, low-energetic charged particles, X-ray fluorescent radiation, and Compton electrons from $\mathrm{X}$-rays and $\gamma$-rays - are expounded by Strüder et al. (2000).

A regular increase of the X-ray background originates in the Van Allen radiation belt. When the satellite passes this zone every 48 hours, the particle flux exceeds the detector limits, and any observation has to be stopped for at least three hours. Erratic fluctuations in the background signal on time scales up to hours occur, when accelerated particles reach the detectors. These so-called "soft protons" most probably have their origin in the magnetospheric reconnection of the particle flux from the active Sun and are channeled to the focal plane by random scattering on the mirrors and the optical elements (Lumb et al. 2002; de Luca \& Molendi 2004). The number of detected single pixel events grows rapidly, and the spectrum steepens exponentially towards lower energies (Strüder et al. 2000, 2001). The vignetting properties deviate considerably from those of the quiescent background, and the intensity falls up to $30 \%$ with increasing off-axis angle. Figure 4.5 shows the influence of solar proton flares 
on the EPIC/pn spectrum and vignetting. The optical blocking filters may attenuate the disturbance of the observation, as the particles lose energy when passing the filters. This is most efficiently reached with the thick filter. As soon as a steep rise in the count rate is detected, the filter wheel is closed to avoid instrumental damage. There is hardly any possibility to distinguish softproton induced events from real X-ray events in science exposures. The soft proton flares may reach intensities of more than 1000 times the quiescent background (Kuntz \& Snowden 2008). Read \& Ponman (2003) describe the soft proton flares as unpredictable in time, spatial, and spectral distribution. The increased background can be identified in light curves of single events with energies above $10 \mathrm{keV}$, where no source signal is expected, or in light curves extracted from definitely source-free ("cheesed") event lists. The usual method to correct for the influence of soft protons consists in the strict removal of the affected time intervals from the analyzed data set. Sufficiently large bad time intervals have to be chosen, as the low-energetic events appear to turn on before the high-energetic ones (Read \& Ponman 2003).

\subsubsection{The Science Analysis Software Package SAS}

The Science Analysis Software Package SAS (Loiseau et al. 2007; Snowden et al. 2008), maintained by the XMM-Newton Science Operations Centre and the Survey Science Centre, manages the extraction of the raw data and its preparation for a further analysis. The observational data are delivered as $O b$ servation Data Files (ODFs), which contain the instrument science, housekeeping, and ancillary data. Pipeline Products from the Survey Science Centre allow for a first inspection of the data. For a thorough re-reduction, the SAS summarizes all the data needed and combines the raw ODFs with the calibration information in the Current Calibration Files (CCF). The production of calibrated event lists includes the removal of "bad" events and the correction for the instrumental and celestial background. In order to identify valid X-ray events and to distinguish them for example from cosmic ray tracks, event patterns have been predefined for the EPIC cameras. An on-board recognition scheme searches for signals in $5 \times 5$ pixel matrices and assigns a pattern number to each recognized event. Figure 4.6 shows a selection of event patterns used for filtering the EPIC imaging and timing data. During the SAS data reduction, the user specifies the valid patterns which are considered as true X-rays and used to filter the EPIC event lists. The standard filtering scheme includes single events for timing mode data, single and double events for EPIC/pn, and 


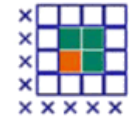

9
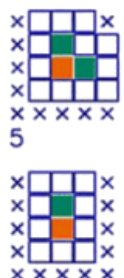

1
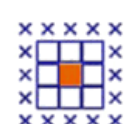

0
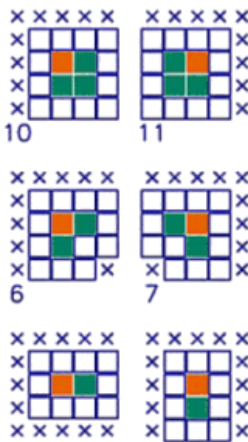

2
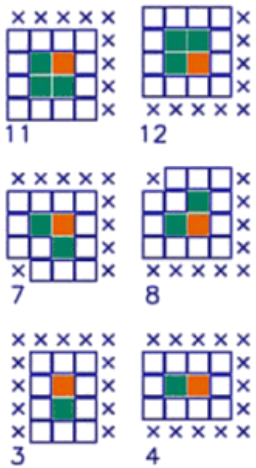

12
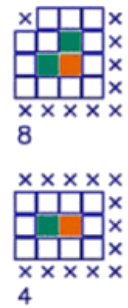

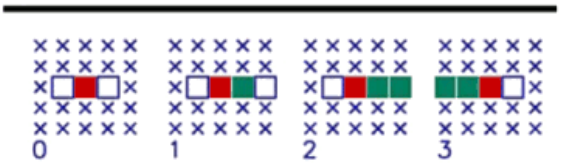

Figure 4.6: Selection of EPIC event patterns for imaging (upper rows) and timing mode (lowest panel), respectively. Pattern number 0 corresponds to single events, $1-4$ to doubles, $5-8$ to triples and $9-12$ to quadruples. The central pixel with the highest energy is marked in red, the other pixels above the threshold in green. White pixels have energies below the threshold, and the crossed pixels can be of all types. Adapted from Loiseau et al. (2007).

single to quadruple events for EPIC/MOS imaging mode data. Pile-up and $\mathrm{X}$-ray loading (Section 4.1.1) may disturb the mapping of the event patterns in different ways: Pile-up lets singles migrate to doubles, whilst in the case of X-ray loading, doubles are registered as singles. Excising the center of the point spread function is the usual method to correct for both the contaminations. On the basis of the filtered event lists, source and background images, light curves, and spectra can be extracted. Filtering expressions provide for a restriction to certain energy intervals, time spans, and/or spatial regions and thus in particular for the creation of phase-resolved spectra or energy-resolved light curves. 


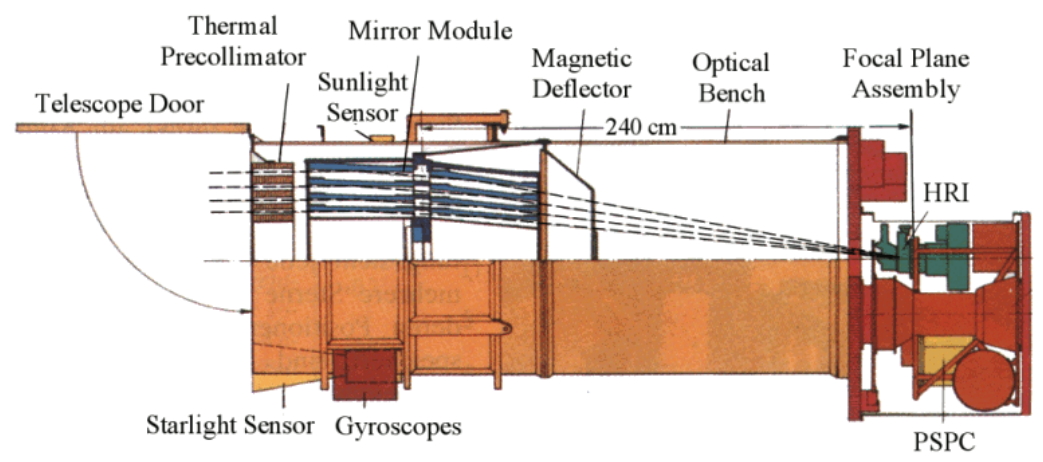

Figure 4.7: Cut through the X-ray telescope XRT on-board ROSAT. The sketch shows the four-fold nested Wolter I mirror assembly to the left, and to the right the detector carousel comprising the three focal plane instruments, two PSPC proportional counters plus the High Resolution Imager HRI. Adapted from Trümper (1990).

\subsubsection{ROSAT}

The ROentgen SATellite ROSAT ${ }^{1}$ (e.g. Trümper 1990), a German-British-US mission, has been launched in June 1990, and operation ceased in February 1999. The first part of the mission, lasting about half a year, was dedicated to an imaging all-sky survey. More than 60000 new X-ray sources of nearly all astrophysical object classes were detected during this time. In the following eight years, pointed observations of selected objects were performed. The scientific payload of ROSAT consisted of the X-Ray Telescope XRT (Figure 4.7), covering the $0.1-2.5 \mathrm{keV}(6-100 \AA)$ spectral range, and the XUV Wide Field Camera WFC $(60-300 \AA / 0.04-0.2 \mathrm{keV})$ mounted co-aligned. The XRT was equipped with a four-fold nested confocal Wolter I mirror assembly and three focal plane instruments: two gas-filled Position Sensitive Proportional Counters PSPC plus the High Resolution Imager HRI, which could be alternately positioned in the focus. The PSPC detectors obtained spectrally resolved $\mathrm{X}$-ray images in four energy bands within a field of view of 2 degrees, reaching a spatial resolution around 25 arcsec and an energy resolution of $\Delta E / E=0.4$ @ $1 \mathrm{keV}$. In contrary, the HRI provided no energy information, but a higher spatial resolution up to 3 arcsec. The WFC camera comprised three nested

\footnotetext{
${ }^{1}$ More information on the ROSAT mission is collected under http: //www. mpe.mpg.de/xray/wave/rosat/index.php.
} 
Wolter-Schwarzschild mirrors, focusing the incoming light onto one of two redundant Microchannel Plate MCP X-ray photo multipliers, and was equipped with eight alternative absorption filters. It features a large 5 degree field of view and an angular resolution of $1 \mathrm{arcmin}$, lower than that one of the X-ray detectors.

Major scientific results from the ROSAT mission have been for instance the X-ray and XUV source catalogs, the detection of isolated neutron stars and of the X-ray emission from comets, the X-ray observation of the impact of the comet Shoemaker-Levy on Jupiter in 1994, and the discovery of soft shadows in the diffuse X-ray background.

\subsection{Probing the XMM-Newton calibration at low energies}

The calibration process of instruments operated on-board X-ray missions commences during the construction phase in laboratories and test facilities. Inflight observations of standard stars with well known properties and crosscalibrations with other instruments help to improve the ground calibration. The current status of XMM-Newton, summarized in Kirsch (2007), benefits from the contributions of a large international calibration team and features inter alia an accuracy of the energy scale of $\pm 10 \mathrm{eV}$ and of the EPIC instrumental effective area of better than $\pm 5 \%$ at energies above $0.4 \mathrm{keV}$. Mainly with the instrumental background rapidly increasing towards the lowest energies, uncertainties remain in the soft energy band, which are described in the calibration documentation and release notes ${ }^{2}$. The steep decline of the effective area below the O-edge at $E=0.53 \mathrm{keV}$ complicates the reconstruction of the instrumental response function. The EPIC/MOS CCDs undergo considerable changes of the energy redistribution with time and with the source position on the CCD, partly hardening and partly softening the spectra below $0.5 \mathrm{keV}$ (Read et al. 2006). These variations are investigated in the calibration process, reducing the time dependence of the residuals to an order of $10 \%$ over the mission lifetime (Stuhlinger et al. 2008).

The in-flight calibration relies on non-variable objects, to which accurate spectral models exist. The pool of standard stars for the soft X-ray range is small, since most of the calibration objects like blazars and supernova remnants

\footnotetext{
2 Available under

http://xmm.vilspa.esa.es/external/xmm_sw_cal/calib/documentation/ and http://xmm.vilspa.esa.es/external/xmm_sw_cal/calib/rel_notes/.
} 
have their flux maxima at higher energies. For the XMM-Newton spectra below $1 \mathrm{keV}$, primarily the isolated neutron star RX J1856.4-3754, the supernova remnant 1ES 0102-72.2, and the O-star $\zeta$ Pup are employed (Kirsch 2007). ${ }^{3}$ Beuermann et al. $(2006,2008)$ present the soft X-ray standards HZ 43 A, Sirius B, and RX J1856.4-3754 from a cross-calibration of Chandra LETG, ROSAT PSPC, and EUVE data, and derive emended model spectra. The results on HZ 43 A and additional data of AM Her allow Beuermann (2008) to determine corrections to the ROSAT PSPC effective area at energies below $0.28 \mathrm{keV}$. Archival XMM-Newton data exist for two of the objects, HZ43 A and RX J1856.4-3754 (summarized in Tables B.1 to B.3). For the use with XMMNewton observations of magnetic cataclysmic variables, where the white dwarf primary shapes the soft part of the spectrum, the calibration of XMM-Newton in the energy range below $1 \mathrm{keV}$ is investigated in the following, describing the soft X-ray standards, the calibration method, and the results.

\subsubsection{Objects}

\subsubsection{The hot white dwarf $\mathrm{HZ} 43 \mathrm{~A}$}

The binary system HZ 43 consists of a DA white dwarf with an optical brightness of $B=12.5$ and of a dimmer nearby dM3.5e secondary. Roughly simultaneously, it was identified as an exceptionally intense EUV source (Lampton et al. 1976; Margon et al. 1976) and as very luminous in soft X-rays (Hearn et al. 1975, 1976), with both the wavelength ranges governed by emission from the white dwarf. As for its spectrum, analyses in different wave bands have shown a considerable depletion of helium and heavier elements (upper abundance limits e. g. by Barstow et al. 1995; Dupuis et al. 1998). The nearly pure hydrogen spectrum qualifies HZ $43 \mathrm{~A}$ as a standard star for optical, ultraviolet, and soft X-ray flux calibration, and thus has been subject to careful spectral modeling. Beuermann et al. (2006, see also Beuermann et al. 2008) establish a hydrogen NLTE model at an effective temperature of $T_{\text {eff }}=51111 \pm 660 \mathrm{~K}$ and a surface gravity of $\log g=7.90 \pm 0.08$ (cgs), compiled with the Tübingen Model Atmosphere Package TMAP (Werner et al. 2003). A concise description of the model and the treatment of interstellar absorption with abundances from Wilms et al. (2000) can be found in their paper (Beuermann et al. 2006).

\footnotetext{
${ }^{3}$ The observations underlying the current calibration are also listed under http://xmm2.esac.esa.int/cgi-bin/ept/preview.pl.
} 
The dM3.5e companion HZ 43 B was identified in optical images at a distance of about $2^{\prime \prime}$ from HZ 43 A (Humason \& Zwicky 1947). M dwarfs are known to emit X-rays, which are softer in the case of inactive stars and harder in the case of activity. Due to the low luminosity, however, Napiwotzki et al. (1993) estimated that the ROSAT instruments were not sensitive enough to measure a potential contribution of HZ 43 B to the total X-ray flux. O'Dwyer et al. (2003) scanned the WGACAT catalog of ROSAT PSPC observations (White et al. 2000) systematically for hard X-ray emitting white dwarfs. They find the predominant part of the HZ 43 flux in the $0.1-0.4 \mathrm{keV}$ band, but about $0.47 \%$ of the PSPC counts at energies above $0.4 \mathrm{keV}$, and assign the hard flux to emission from the secondary star. Beuermann et al. (2006) confirm the indication of a weak hard X-ray component and approximate it by bremsstrahlung at $k T_{\text {brems }}=0.6 \mathrm{keV}$.

\subsubsection{The isolated neutron star RX J1856.4-3754}

RX J1856.4-3754 (shortly RX J1856) is a member of the so-called magnificent seven, a sample of nearby isolated neutron stars with thermal spectra, discovered or confirmed in ROSAT observations (see Haberl 2007 for a review). Its classification as the X-ray brightest isolated neutron star dates from Walter et al. (1996). Van Kerkwijk \& Kulkarni (2001) report on an H $\alpha$ nebula with comet-like morphology around RX J1856, which they have identified in optical photometry and spectroscopy. The puzzling lack of X-ray pulsations, otherwise seen in the majority of the isolated neutron stars (Haberl 2007), has been settled with the discovery of weak $7.055 \mathrm{~s}$ pulsations in XMM-Newton EPIC light curves by Tiengo \& Mereghetti (2007). Two characteristics qualify RX J1856 as a standard star for soft X-ray calibrations: its smooth X-ray spectrum and its optical faintness $\left(V \sim 27^{\mathrm{m}} 7\right)$, obviating a contamination of the $\mathrm{X}$-ray spectra by low-energy photons. Thus, the International Astronomical Consortium for High Energy Calibration (IACHEC ${ }^{4}$ ) aims to establish RX J1856 as a soft $\mathrm{X}$-ray standard. The thermal spectrum, free of any features besides the hydrogen series at longer wavelengths, is excellently described by two black bodies at temperatures between $30 \mathrm{eV}$ and $65 \mathrm{eV}$, one probably representing a heated spot, one the neutron-star surface (Burwitz et al. 2003). Based on a crosscalibration of Chandra and ROSAT spectra, Beuermann et al. (2006) derive a two black body model, attuned to the optical flux, at $k T_{\mathrm{bb}, 1}=62.83 \pm 0.41 \mathrm{eV}$ and $k T_{\mathrm{bb}, 2}=32.26 \pm 0.72 \mathrm{eV}$. This fit yields an interstellar absorption of

\footnotetext{
${ }^{4}$ http://www.iachec.org/index.html
} 
$N_{\mathrm{H}}=8.91 \pm 0.37 \cdot 10^{17} \mathrm{~cm}^{-2}$, when assuming a hydrogen ionization degree of $x_{\mathrm{H}}=0.25$. The XMM-Newton calibration team employs RX J1856 as a calibration target for the soft energy range and models the X-ray spectrum with a single absorbed black body at $N_{\mathrm{H}}=5.7 \pm 0.7 \cdot 10^{20} \mathrm{~cm}^{-2}$ and $k T_{\mathrm{bb}}=63.7 \pm 0.03 \mathrm{eV}$ (Stuhlinger et al. 2008).

\subsubsection{Data selection and method}

Using SAS v8.0 together with the most recent calibration files (July 2008 release), we test the precision of the flux calibration and derive empirical effective area corrections on the soft objects HZ 43 A and RX J1856. The EPIC spectra are cross calibrated against Chandra and ROSAT PSPC data by means of the Beuermann et al. (2006) photon spectra, which are accurate up to $\pm 5 \%$ at energies above $0.25 \mathrm{keV}$.

Two observations of HZ 43 and twelve of RXJ1856 are listed in the XMMNewton data archive, most of them in small window mode and thin filter (see Tables B.1 to B.3). We extract the EPIC spectra from extended circular source regions with radii between 1 and 2 arcmin in the case of HZ 43 and between 25 and 75 arcsec in the case of RX J1856, depending on the spatial extent of the objects on the CCD in the individual exposures. If possible, the background regions are located on the same chip as the source. For the MOS data in small window mode, we have selected source-free background areas on an outer CCD. Four effects possibly deteriorate the data quality and had to be considered in the data reduction: soft proton flares, optical and X-ray loading, and pile-up. The high X-ray flux of both the sources notwithstanding, a restrictive flare screening was performed in order to achieve a high data quality. Optical and X-ray loading as well as pile-up (Section 4.1.1) may affect the soft energy range strongly and appear most strikingly in spectra with a steep shape (Ehle et al. 2007). We have calculated the pile-up probability for each exposure and checked the single and double event fractions as given by the EPATPLOT task in different energy bands. All observations in full frame mode suffer considerably from pile-up; to a minor degree also the large window data. From the HZ 43 exposures, inner circles with radii between 20 and 75 arcsec have been excised in order to reduce the pile-up probability to values below $5 \%$.

For investigating the low energy calibration, we consider the EPIC spectra over all channels and waive the low energy cutoff in the EPCHAIN task. EPREJECT, aiming at a more precise reconstruction of the EPIC/pn energy re- 


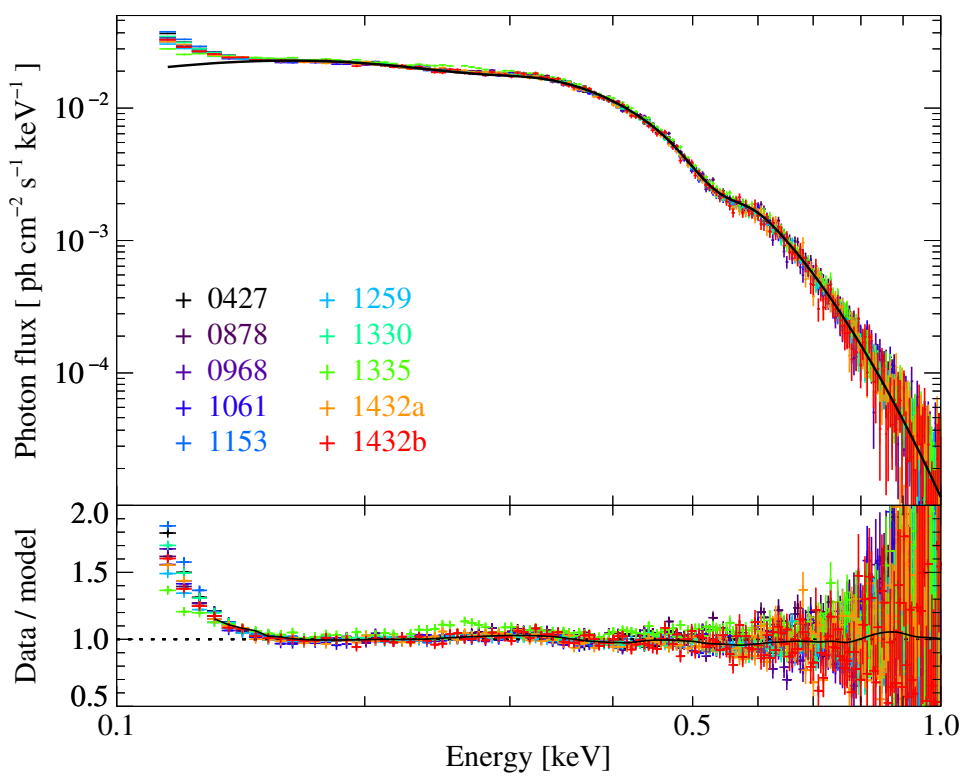

Figure 4.8: Flux stability of the RX J1856 EPIC/pn spectra. The plot shows the data from all the exposures in small window mode and thin filter between April 2002 and October 2007. Upper panel: Data points plus the Beuermann et al. (2006) model as described in the text (black line). Lower panel: Data-to-model ratio. Over-plotted is the resulting correction function (see Section 4.2.3.1). The 2007 March 25 observation (rev. 1335, green) deviates from the mean by several per cent for unknown reasons.

distribution in the softest range, is only available for data in full frame and in timing mode and therefore has not been performed. The unbinned spectra jointly are compared to the photon spectra of Beuermann et al. (2006), adopted for the use within XSPEC in the form of ATABLE additional models. The datato-model ratios reveal systematic deviations on an energy scale of several ten electron volts, which we attribute to uncertainties in the effective area determination, thus assuming them to be independent from the incident energy in good approximation. We derive a correction function to the instrumental effective area from the error weighted mean of the data-to-model ratios, smoothed with a three-point boxcar to get rid of statistical small scale fluctuations. An additional smoothing of the noisier parts of the spectra at energies above $0.8 \mathrm{keV}$ guarantees a continuous transition to the higher energy range, where the cor- 
rection function is set to one. With respect to the model accuracy (Beuermann et al. 2006), we add a systematic uncertainty of $5 \%$ to the weighted statistical errors derived from the averaged observations. The correction is directly applied to the spectrum generated within SAS v8.0, so the response function is calculated with the RMFGEN and ARFGEN tasks as usual. Finally, the low energy cutoff, elsewise defined within the SAS tasks, is chosen as the energy below which the standard star spectra show an increasing variation.

\subsubsection{Results}

\subsubsection{RX J1856.4-3754: EPIC/pn thin filter}

The EPIC/pn flux of RXJ1856 is stable to a high degree over all exposures (Figure 4.8), which cover a time span of more than five years. The 2007 March 25 observation (rev. 1335) forms a sole exception with its flux exceeding the mean by about $5 \%$. The reason for this discrepancy is unclear, but it might correlate with the source position on the chip, off-axis by 2 arcmin (Stuhlinger et al. 2008). Data and model agree well within the usable energy range between $0.18 \mathrm{keV}$ and $1.0 \mathrm{keV}$. The effective area correction of up to $\pm 5 \%$ (Figure B.1) lowers the reduced $\chi^{2}$ of the joint fit to the unbinned data from $\chi_{\text {red,i }}^{2}=1.59$ to $\chi_{\text {red,f }}^{2}=1.30$. For the binned spectra with a minimum of 20 counts per bin, diminishing the noise at shorter wavelengths, the joint fit yields $\chi_{\text {red }}^{2}=1.18$. The $\chi_{\text {red }}^{2}$ of the individual spectra improve by up to 0.75 . All the observations in use are listed in Table B.1.

\subsubsection{RX J1856.4-3754: EPIC/MOS thin filter}

The calibration of the XMM-Newton EPIC/MOS data is complicated by the energy redistribution function changing with the observation date (Kirsch 2007). In order to minimize potential systematic errors arising from the time dependence, only observations after September 2005 (rev. 1061) have been chosen for the calibration test (cf. Tables B.2 and B.3). They differ only little from each other and are close to the observation epochs of the polars AI Tri and QS Tel presented in this work. The MOS1 spectra conform with the Beuermann et al. (2006) photon spectra acceptably well, resulting in a higher overall $\chi^{2}$ than the EPIC/pn data. The adjustment of the MOS1 effective area reaches a maximum amplitude of $7 \%$ (Figure B.2), while the reduced $\chi^{2}$ shrinks from $\chi_{\text {red,i }}^{2}=2.4$ 


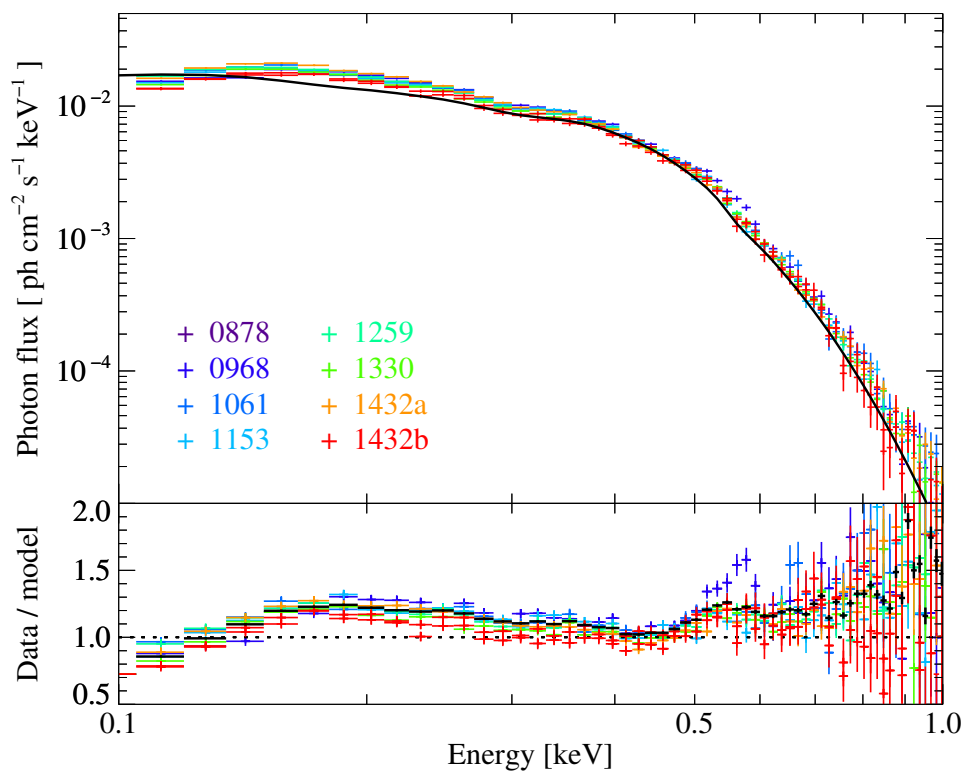

Figure 4.9: EPIC/MOS2 data of RX J1856 in small window mode and thin filter taken between September 2004 and October 2007 and the Beuermann et al. (2006) model (black line). The lower panel shows the corresponding data-to-model ratios with black crosses marking their weighted mean.

to $\chi_{\text {red,f }}^{2}=1.6$ in the $0.18-1.0 \mathrm{keV}$ range. The MOS2 spectra diverge from the model more strikingly, by as much as $20 \%$, with the consequence of a high $\chi_{\text {red,i }}^{2}=11$. A trend in the data-to-model ratios towards values larger than one may indicate a systematic underestimate of the MOS2 flux (Figure 4.9). The revision of the soft band at energies below $1.0 \mathrm{keV}$ results in an adequate joint fit with $\chi_{\text {red,f }}^{2}=1.7$ (Figure B.3), but leaves the question open, whether flux adjustments were necessary also at higher energies. As for the energy range to be used in the data analysis, a low-energy cutoff at $E_{\min }=0.18 \mathrm{keV}$ is recommended for both the MOS instruments as the most restrictive limit, but the reconstruction of the energy redistribution may also do well at lower energies down to $E_{\min }=0.1 \mathrm{keV}$. 


\subsubsection{Interpolation to the medium filter}

The EPIC instruments on-board XMM-Newton are equipped with identical filter wheels, carrying four filters each (Section 4.1.1). The thin and the medium filters consist of a $1600 \AA$ thick poly-imide film, onto which aluminum layers of $400 \AA$ and $800 \AA$, respectively, are evaporated (Turner et al. 2001). The properties and transmission function of the thick filter, composed of polypropylene, aluminum, and tin, deviate from the other filters to a larger degree. In order to account for the filter characteristics and for the number of optically generated photons which can pass the filter, individual response matrices and effective areas are calculated for each configuration.

The majority of the archival RXJ1856 EPIC observations have been performed using the thin filter. We confine the adjustment of the calibration to the effective area and thus consider it to be essentially independent of the incident energy. The correction functions therefore reflect but the instrumental properties and should basically not dependent on the chosen filter. If so, the relation between the medium and the thin filter correction functions is directly correlated with the relation between the medium and the thin filter data itself. In order to derive the effective area correction for medium filter data, we fold the energy redistribution matrix with the instrumental effective area for each observation in thin filter and for the 2004 September 24 MOS exposures in medium filter (listed in Tables B.2 and B.3). The ratio between the medium filter and the mean thin filter responses, multiplied with the correction functions as derived in Sections 4.2.3.1 and 4.2.3.2, gives us the adjustments to the effective area for medium filter observations.

The same approach can be used to transfer the correction functions to data obtained in the large window and full frame modes. It is recommended to skip the SAS EPREJECT task during the extraction of full frame data, if the correction functions shall be applied. Large window observations of RX J1856 are available with both the MOS cameras on April 15, 2005 (rev. 0980). The correction functions, adjusted to the corresponding instrumental response, reduce the $\chi^{2}$ of the fits to these data sets considerably, validating the modifications to the effective area. Like the EPIC/pn data, the MOS observations on March 25, 2007 (rev. 1335) exhibit an increased source flux and thus cannot be consulted for comparison. The deviations between the correction functions derived from data that have been obtained in different frame modes lie below $1 \%$. 


\subsubsection{HZ43 A}

HZ 43 A, successfully used in the cross-calibration of ROSAT PSPC with Chandra data by Beuermann et al. (2006) and Beuermann (2008), shows an $\mathrm{X}$-ray bright and steep spectrum up to $0.28 \mathrm{keV}$, and therefore has been considered as a possible standard star for the lowest energy range. It demonstrates the fundamental difficulty in cross-calibrating Chandra and XMM-Newton spectra: Chandra requires $\mathrm{X}$-ray bright objects to obtain a high signal-to-noise, while high X-ray fluxes cause strong pile-up effects in the XMM-Newton EPIC data. Due to the brightness of HZ 43 over all the wave bands, optical and X-ray loading can additionally deform the EPIC spectra. Both the effects show up and are too strong to overcome them by excising an inner part of the source region in the EPIC/pn data. The EPIC/MOS spectra, less susceptible to distortion by pile-up, appear to be more reliable. A comparison with RX J1856 confirms the effective area corrections derived for MOS1 and MOS2 in Section 4.2.3.2.

The EPIC spectra of HZ 43 A show evidence of harder source photons as proposed by O'Dwyer et al. (2003) and Beuermann et al. (2006). The number of background subtracted source counts above and below $0.28 \mathrm{keV}$ relates as $150: 1$. The flux of the harder component is too low to analyze its spectral shape in detail.

\subsubsection{Conclusions}

Probing the calibration of the EPIC instruments in the soft energy range between 0.18 and $1.0 \mathrm{keV}$, we find it to be accurate within $\pm 5 \%$ and $\pm 7 \%$ for EPIC/pn and MOS1 respectively, whereas the MOS2 data of RX J1856 deviate by up to $20 \%$ from the Beuermann et al. (2006) model. The correction functions to the instrumental effective areas flatten the systematic residuals, in particular around the $0.37 \mathrm{keV} \mathrm{C} \mathrm{VI} \mathrm{line.} \mathrm{They} \mathrm{primarily} \mathrm{apply} \mathrm{to} \mathrm{boresight} \mathrm{ob-}$ servations of objects with soft X-ray spectra like polars, since they may depend on the incident photon energy and on the location of the source on the chip. By our employing an absorbed two black body model according to Beuermann et al. (2006) for RX J1856, the S-shape residuals which remain in the single black body fit of the XMM-Newton calibration team (Stuhlinger et al. 2008) vanish. With systematic uncertainties on the order of $7 \%$ at energies below $0.8 \mathrm{keV}$, adjusting the effective area can significantly improve the fit quality in the softest energy band of white dwarfs and polars. 


\section{CHAPTER 5}

\section{The long-period polar AI Tri}

AI Tri (RX J0203.8+2959) was first described within a sample of ROSATdiscovered bright soft X-ray sources by Beuermann \& Thomas (1993). Their classification of AI Tri as an AM Her type binary (also called polar) was later on confirmed in a multi-wavelength study by Schwarz et al. (1998), who identified cyclotron humps in optical spectra obtained during a high state of the system. Its orbital period of $P_{\text {orb }}=4.6 \mathrm{hrs}$ is one of the longest known among polars, whereas the magnetic field strength of $B=38 \pm 2 \mathrm{MG}$ and the amplitude of the long-term brightness variations between $V=18^{\mathrm{m}} \cdot 0-15{ }^{\mathrm{m}} 5$, detected by Schwarz et al. (1998), lie in the typical parameter range of this class. Multicolor photometry presented by these authors reveals the phase dependence of the light curve minima and possible optical quasi-periodic oscillations (QPOs) with periods between 6.5 and 14 min. Katajainen et al. (2001) use photopolarimetric $U B V R I$ observations during intermediate accretion states to further constrain the accretion geometry. Based on the wavelength dependence of the light curve minima and the variations in both the linear and the circular polarization, Katajainen et al. (2001) suggest a high inclination of $i \sim 70^{\circ} \pm 20^{\circ}$ and accretion onto two almost equally fed magnetic poles, while Schwarz et al. (1998) prefer the model of a single dominating accretion region. Since its identification by Beuermann \& Thomas (1993), AI Tri is known to belong to the number of AM Her systems that show an evident dominance of soft over hard $\mathrm{X}$-ray emission. With its distinct soft X-ray flux, it is a valuable object to test the picture of the energy balance between hard post-shock and soft reprocessed component in MCVs. 


\subsection{Observations and data reduction}

\subsubsection{XMM-Newton}

With XMM-Newton, we obtained a $20 \mathrm{ksec}$ exposure during a high state of AI Tri on August 22, 2005 (archived under observation ID 0306841001), corresponding to more than one binary orbital cycle. All observations with the EPIC instruments were performed in full frame mode with the thin filter. Simultaneously, the UV light curve at an effective wavelength of $2310 \AA$ has been measured with the Optical Monitor $(\mathrm{OM})$ used in timing mode and the UVM2 filter. RGS spectra are not available from this pointing, since the X-ray flux of the object was too low. Due to technical problems, a first attempt in the same configuration on August 15, 2005 resulted in partially corrupted data (archived under observation ID 0306840901). During 4 to $5 \mathrm{ksec}$ of this exposure, EPIC/MOS, RGS (1st order), and OM data are usable in addition to $1.1 \mathrm{ksec}$ with EPIC/pn. An overview of all the data obtained is given in Table 5.1.

Light curves, spectra, and images have been extracted making use of the XMM-Newton Software Analysis System SAS v8.0, dividing the source spectra by the adjusted effective area as described in Section 4.2. Single and double events have been processed in the case of EPIC/pn, supplemented by quadruple events for MOS. Source data were collected from an aperture of 25 arcsec around the source center, background data from a larger source free region as close to the source position as possible, which then was scaled and subtracted from the source data. When extracting the mean spectrum and phase-resolved spectra, we have excluded the innermost 5 arcsec of the source region during the intervals of highest count rate to diminish potential pile-up, which can also arise in the case of moderately bright sources when a high background signal is detected (cf. Section 4.1.1). For the EPIC/pn data on August 15, 2005, we extended the source region to $30 \operatorname{arcsec}$ and excised the innermost 7 arcsec in order to account for pile-up effects. Appropriate spectral response matrices have been generated applying the most recent calibration files (July 2008 release) for the individual instruments with the thin filter. EPIC and OM timing data have been barycenter corrected and binned to $10 \mathrm{~s}$ time intervals. Spectral bins comprise a minimum of 20 counts, and a minimum of 30 counts in the case of the mean EPIC/pn spectrum on August 22, 2005. 


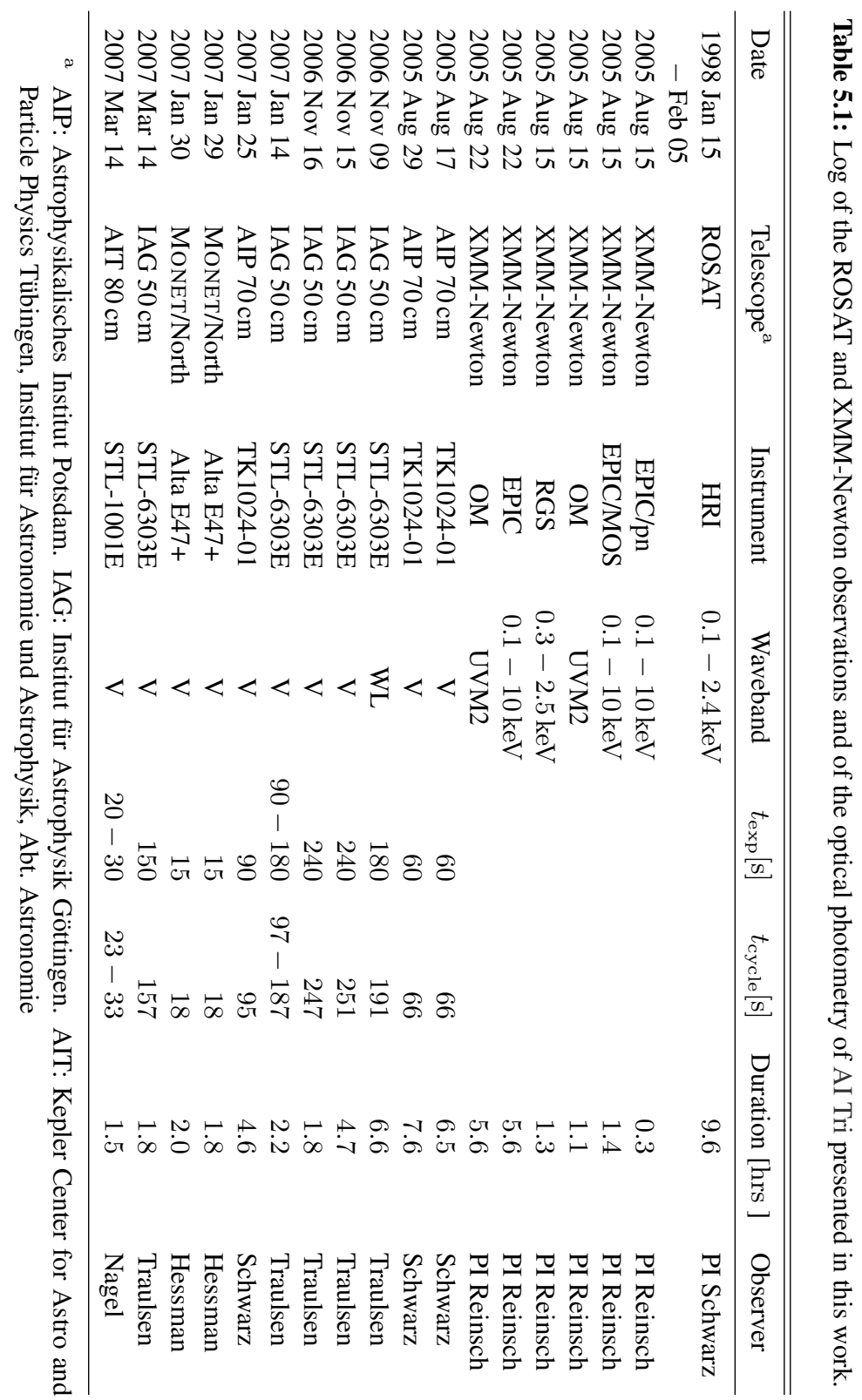




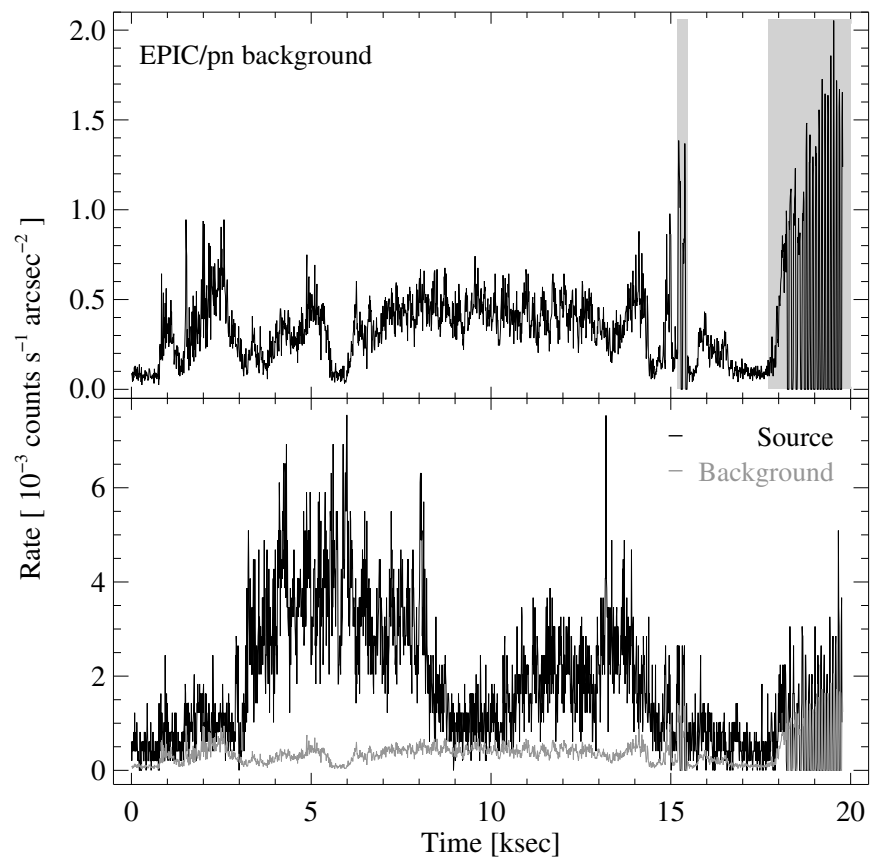

Figure 5.1: Background and source light curves of the $20 \mathrm{ksec}$ XMM-Newton observation of AI Tri with a time binning of $10 \mathrm{~s}$. Top: EPIC/pn background light curve. Intervals excluded from the analysis are marked in light grey. Bottom: Comparison between the EPIC/pn source (black) and background (grey) light curves. The source signal clearly dominates the background during most of the integration time.

\subsubsection{Dealing with the flaring background}

Large parts of the $20 \mathrm{ksec}$ exposure on August 22, 2005 suffer from soft proton flares, excited by high solar activity (Section 4.1.1.1). Figure 5.1 shows the uncorrected source and background light curves of AI Tri, and Figure 5.2 the $\mathrm{X}$-ray background above $130 \mathrm{keV}$ on August 21 and 22, 2005 in two different energy ranges as measured by the XMM-Newton Live Radiation Monitor ${ }^{1}$. In addition to the enhanced proton flux, the satellite nears the Van Allen radiation

\footnotetext{
${ }^{1}$ The XMM-Newton Live Radiation Monitor data are available at http://xmm2.esac.esa.int/external/xmm_obs_info/radmon/.
} 

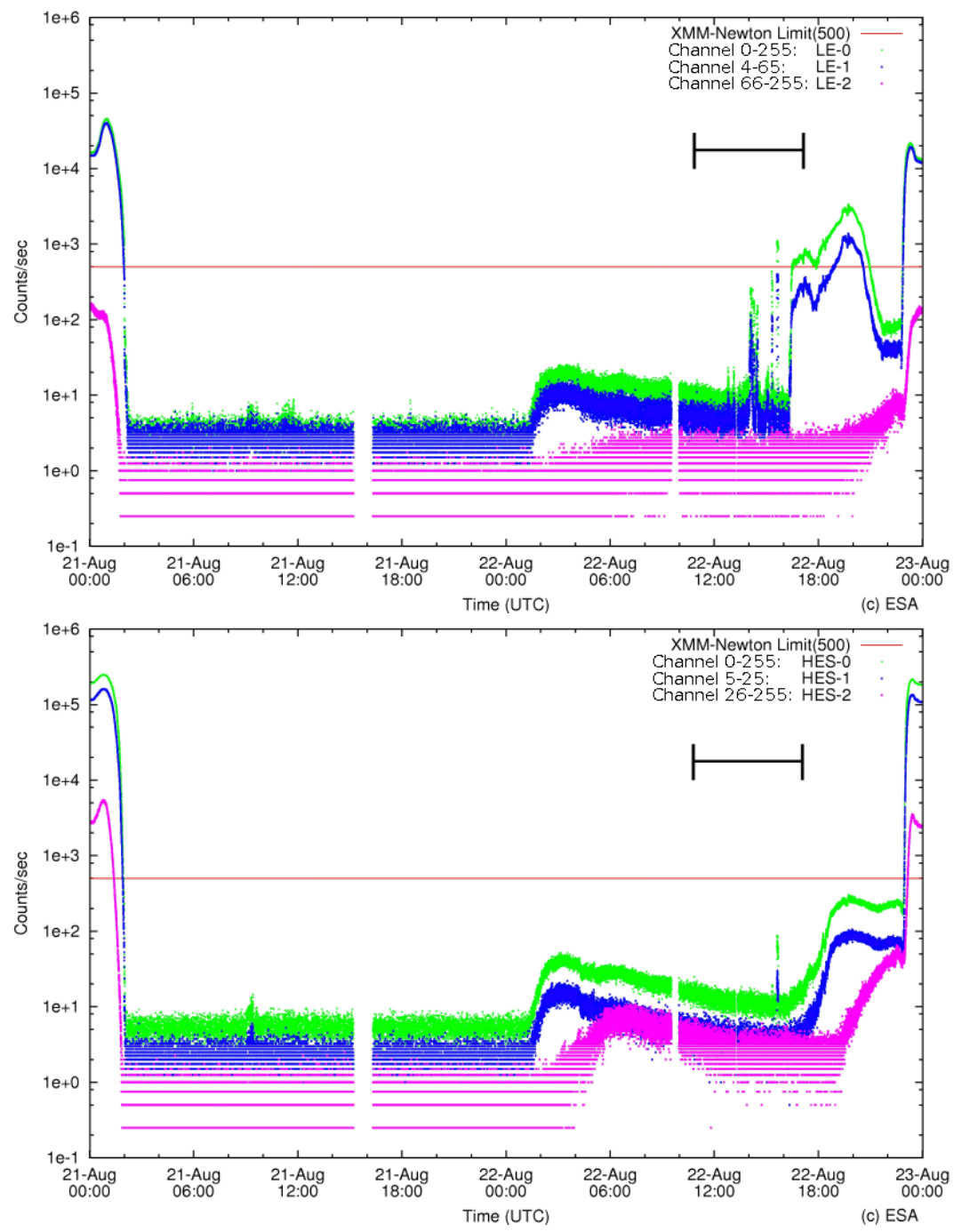

Figure 5.2: Background activity as measured with the XMM-Newton radiation monitor on August 21 and 22, 2005. Upper panel: Electron count rates at $130 \mathrm{keV}-1.5 \mathrm{MeV}$ and protons at $1-4.5 \mathrm{MeV}$ in different channels. Lower panel: Electrons at $1.0-1.75 \mathrm{MeV}$ and protons at $8-40 \mathrm{MeV}$. The observation interval is marked in black. Adapted from http://xmm2.esac.esa.int/external/xmm_obs_info/radmon/. 
belt towards the end of the observation time. Two approaches help to check for flaring events in the observational data of a highly variable source. Modulations of the light curves extracted for energies above $10 \mathrm{keV}$, where no source signal is expected, indicate the presence of an enhanced soft-proton background. The behavior of the flares in different energy bands can be evaluated in background light curves from source-free event lists. These energy-resolved background light curves are crucial for a restrictive proton flare screening, as the low-energy flares typically turn on earlier than the high-energetic ones (Read \& Ponman 2003).

In order to test the data quality, temporal, spatial, and spectral variability of the flaring background have been analyzed and checked against the nonvariable object RXH J020343.2+300506 in the FOV. Light curves, spectra, and images, extracted from twelve different background regions during intervals of high and low flaring activity respectively, have been compared and crosscorrelated. Following results can be stated:

1. Spatial variability is negligible on sufficiently small scales, so the background that shall be subtracted from the source signal can be extrapolated from a region close to the source (cf. Figure 4.5).

2. The background influences the light curves only marginally compared to the count rate of the source (Figure 5.1).

3. Proton flares reaching more than 100 times the intensity of the quiescent background are not completely eliminated during the data reduction and leave an increased number of high energy counts in the spectrum.

4. The spatially resolved background spectra are nearly identical within their error bars above energies of $0.2 \mathrm{keV}$. Though generally varying unpredictably from observation to observation (de Luca \& Molendi 2004), the spectral shape remains essentially constant during the $20 \mathrm{ksec}$ pointing.

5. In addition to the spectral steepening at soft energies (Figure 4.5), a high-energy tail arises from the flaring events in the EPIC spectra above $3-4 \mathrm{keV}$.

Images, light curves, and spectra of the non-variable comparison source RXH J020343.2+300506, extracted with respect to the caveats, are obviously 
cleaned from the soft proton flaring events. Owing to the comparatively high signal of AI Tri, an adequate data quality is reached. Only a strong flare after about $15 \mathrm{ksec}$ and the last $10 \%$ of the observation, where the satellite nears the Van Allen radiation belt, need to be excluded from the analysis. Our thorough treatment of the data still cannot guarantee that the proton flaring signal is completely eliminated from the spectra. The potential remaining traces of the flaring events in the spectrum have to be taken into account during the data analysis.

\subsubsection{ROSAT HRI}

In addition to the XMM-Newton observations, we use unpublished archival data obtained between January 15 and February 5, 1998 with the ROSAT HRI detector. The total integration time of $35 \mathrm{ksec}$ is split into nine observation intervals. The individual light curves have been extracted with standard tasks of the EXSAS/MIDAS (Zimmermann et al. 1993) software packages, barycenter corrected, and combined to a common light curve profile.

\subsubsection{Optical observations}

At the Göttingen $50 \mathrm{~cm}$, the Potsdam $70 \mathrm{~cm}$, the Monet/North (Texas) $1.2 \mathrm{~m}$, and the Tübingen $80 \mathrm{~cm}$ telescopes, we have carried out optical $V$-band and white-light photometry during ten nights between August 2005 and March 2007. AI Tri was found in high states or intermediate high states of accretion at an estimated brightness between $V=17^{\mathrm{m}} 0-15^{\mathrm{m}} 5$ during the observations. For data reduction and differential photometry, we use TRIPP (Schuh et al. 2003) and ESO-MIDAS routines. Light curves have been acquired by aperture photometry on the basis of the same reference stars for all the data sets, as far as possible with respect to the different fields of view of the individual instruments. Apparent magnitudes of the system have been calculated against the $V$-band magnitudes of the comparison stars USNO-B1.0 1199-0026710 $\left(V=13^{\mathrm{m}} \cdot 29 \pm 0 .{ }^{\mathrm{m}} 05\right)$ and USNO-B1.0 1199-0026672 $\left(V=14^{\mathrm{m}} 62 \pm 0.05\right)$ determined by Schwarz (1996). Fluxes are given relative to their orbital mean, and flux errors have been derived by means of photon statistics. We correct the times heliocentrically to obtain the minima timings, and to the barycenter on the basis of the JPL ephemeris (Standish 1998), respectively, for comparability with the XMM-Newton data. 


\subsection{Photometry}

\subsubsection{XMM-Newton X-ray}

From our $20 \mathrm{ksec}$ XMM-Newton exposure, we have extracted the first continuous X-ray light curves of AI Tri over a full binary orbit (Figure 5.3b-d). Two $\mathrm{X}$-ray bright phases during the photometric phase intervals $\varphi_{\text {phot }}=0.05-0.40$ and $\varphi_{\text {phot }}=0.50-0.80$, as defined by the ephemeris given in Equation 5.1, commence with a steep rise at $\varphi_{\text {phot }}=0.05$. The flux variation is most pronounced in the soft energy band with the bright phases lasting about $70 \%$ of the orbital period, whereas hard X-ray emission remains visible at a low level incessantly. The hard X-ray flux increases by a factor of about three at the beginning of the first bright soft phase and slightly varies during the second one. The shape of the soft X-ray light curves is characterized by high variability on time scales down to less than one minute, as typical of soft polars. Figure 5.5 shows details of the intensity maxima around photometric phases 0.2 and 0.7 , binned to time intervals of $10 \mathrm{~s}$. Although no regular or periodic pattern is detectable, the rapid variations are obvious.

The associated hardness ratio $\mathrm{HR}=(\mathrm{H}-\mathrm{S}) /(\mathrm{H}+\mathrm{S})$, where $\mathrm{H}$ and $\mathrm{S}$ represent the counts at energies above and below $0.5 \mathrm{keV}$ respectively, correlates largely with the light curves in the soft energy band, tending to 1 during low and to -1 during high soft emission phases (Figure 5.3d). Throughout the soft minimum at $\varphi_{\text {phot }}=0.45$, the hardness ratio levels off around zero and thus differs from the behavior in the low flux range between photometric phases 0.80 and 1.05 , where it rises to an average value of 0.7 . Similar characteristics of the light curves are found from the archival ROSAT HRI data obtained between January 15 and February 5, 1998 (Figure 5.3a).

\subsubsection{XMM-Newton UV}

The observation with the XMM-Newton Optical Monitor and UVM2 filter at an effective wavelength of $2310 \AA$, carried out simultaneously with the EPIC instruments, provides the first mid ultraviolet light curve of AI Tri and spans more than one rotational cycle, interrupted only by four small gaps between the individual exposures (Figure 5.4b). The modulation of the UV flux has a roughly similar shape but a larger amplitude than the optical light curves during high states of accretion presented in Section 5.2.3. Dominated by an almost sinusoidal variation with the orbital period, it shows an additional sharp dip in the light curve minimum near $\varphi_{\text {phot }}=0.0$, slightly shifted in phase against the 


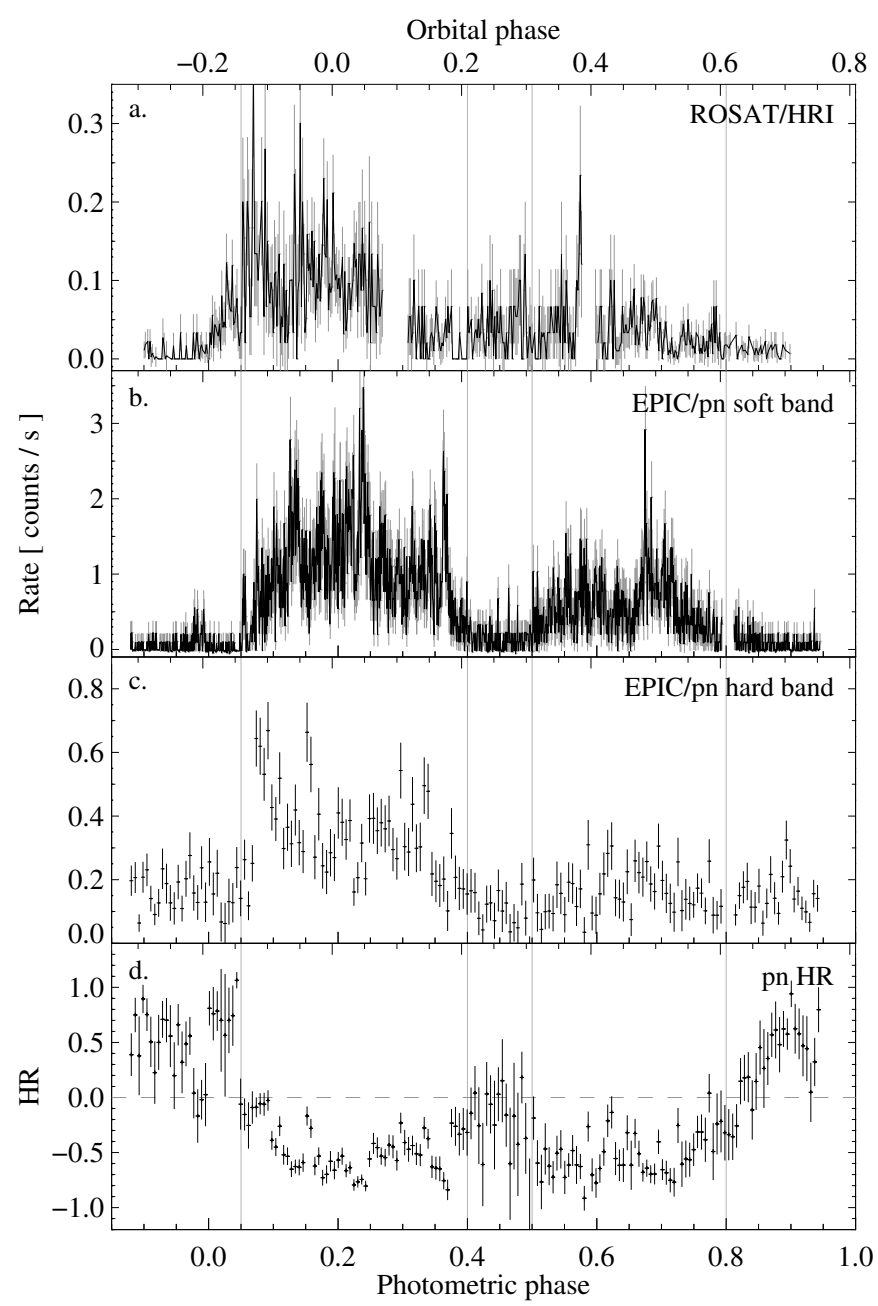

Figure 5.3: X-ray light curves of AI Tri. $a$. Archival ROSAT HRI data of Jan/Feb. 1998 with a total integration time of about $35 \mathrm{ksec}$, combined to a common light curve profile and rebinned to $30 \mathrm{~s}$ intervals. $b$ - $d$. $20 \mathrm{ksec}$ EPIC/pn light curve on August 22, 2005 split into the soft component $\mathrm{S}(E \leq 0.5 \mathrm{keV})$, the hard component $\mathrm{H}(E \geq 0.5 \mathrm{keV})$, and the associated hardness ratio $\mathrm{HR}=(\mathrm{H}-\mathrm{S}) /(\mathrm{H}+\mathrm{S})$. The soft band data have been binned to $10 \mathrm{~s}$, the hard band and the hardness ratio to $100 \mathrm{~s}$ intervals. 


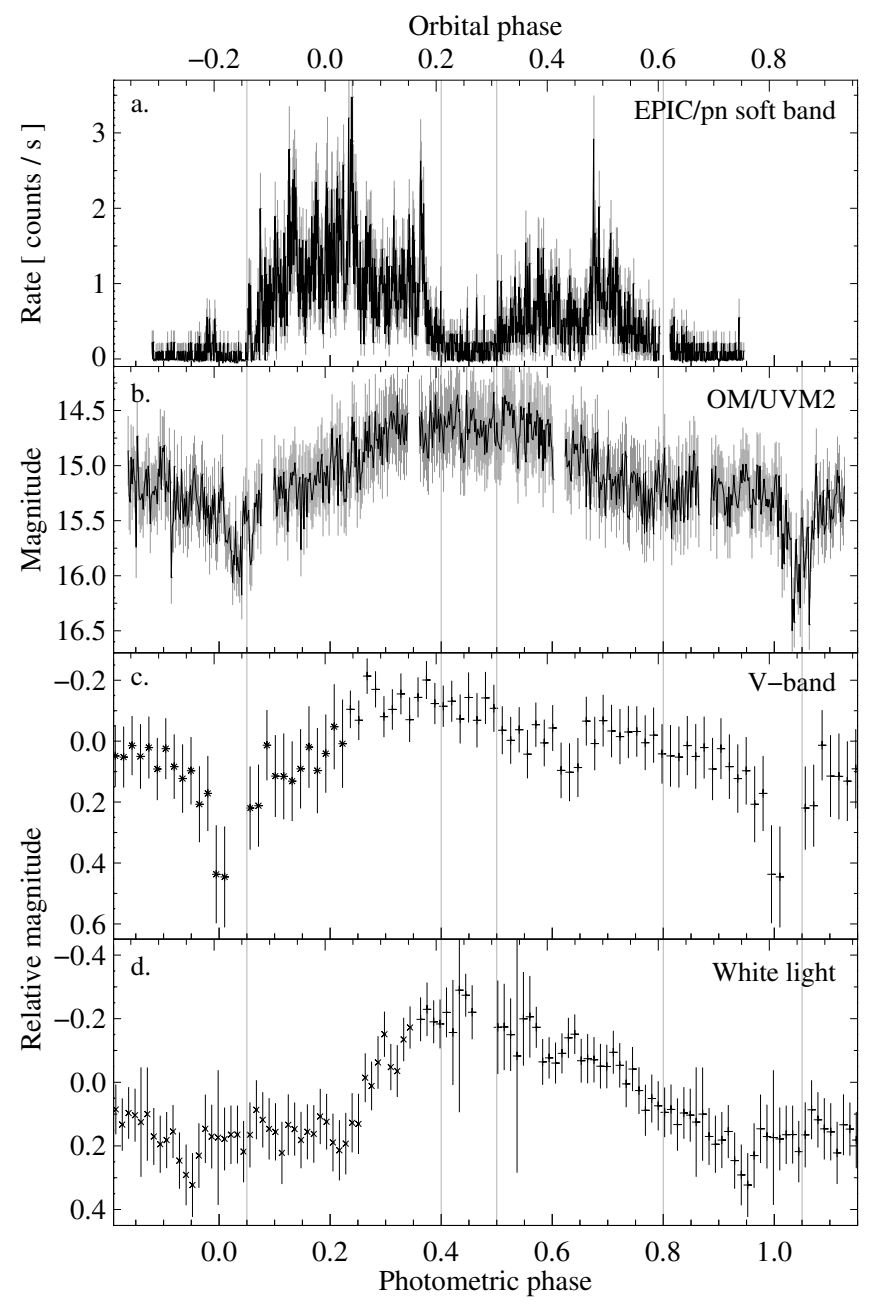

Figure 5.4: $X$-ray, ultraviolet OM, and optical $V$-band light curves of AI Tri, folded over photometric phase on the basis of the ephemeris given in Equation 5.1. a. Soft component $\mathrm{S}(0.1 \mathrm{keV} \leq \mathrm{E} \leq 0.5 \mathrm{keV})$ of the 2005 August $22 \mathrm{EPIC} / \mathrm{pn}$ light curve. $b$. OM light curve in the ultraviolet UVM2 filter at an effective wavelength of $2310 \AA$ in time bins of $30 \mathrm{~s} . c-d$. Optical data obtained at the Göttingen $50 \mathrm{~cm}$ telescope on November 15 and 9, 2006 and plotted twice for clarity. 


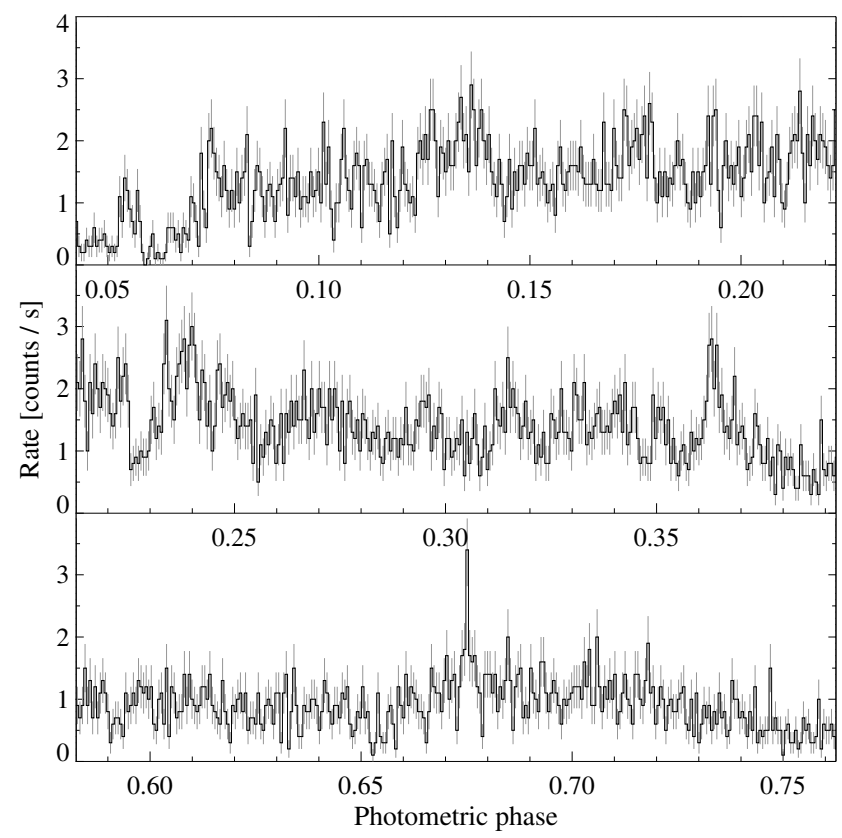

Figure 5.5: Short-term variability in the 2005 August 22 EPIC/pn light curve of AI Tri, binned at a time resolution of $10 \mathrm{~s}$, showing rapid, irregular flickering. Error bars are over-plotted in grey.

optical minimum dip (Section 5.2.3). The maximum at $\varphi_{\text {phot }}=0.45$ appears to be flattened, but due to the lack of further UV data it remains unclear if it is a recurring or an unique feature.

\subsubsection{Optical}

The optical $V$-band light curves of AI Tri (e.g. Figure 5.4c) during high and intermediate high states of accretion are largely consistent with the optical photometry presented by Katajainen et al. (2001) and Schwarz et al. (1998). The orbital modulation deviates from a sinusoidal variation and has a slightly asymmetric shape with steeper rise and smoother decline. As a new feature, an irregular pattern of narrow small dips is superposed with one dip recurring near photometric phase zero. This dip was not seen in earlier optical data. 
Table 5.2: Heliocentric timings of the $V$-band minima used for the determination of the improved ephemeris.

\begin{tabular}{ccrr}
\hline $\mathrm{HJD}\left(T_{\min }\right)$ & $\Delta T_{\min }$ & \multicolumn{1}{c}{$O-C$} & \multicolumn{1}{c}{ Cycle } \\
\hline $49243.93769^{\mathrm{a}}$ & 0.0008 & 0.0246 & -11448 \\
$50042.36460^{\mathrm{a}}$ & 0.0017 & 0.0109 & -7284 \\
$50043.51433^{\mathrm{a}}$ & 0.0009 & 0.0070 & -7278 \\
$50046.39873^{\mathrm{a}}$ & 0.0029 & 0.0498 & -7263 \\
$50047.54640^{\mathrm{a}}$ & 0.0014 & 0.0352 & -7257 \\
$50049.26561^{\mathrm{a}}$ & 0.0013 & 0.0013 & -7248 \\
$50049.45623^{\mathrm{a}}$ & 0.0011 & -0.0046 & -7247 \\
$50122.32779^{\mathrm{a}}$ & 0.0019 & 0.0379 & -6867 \\
$50123.28184^{\mathrm{a}}$ & 0.0013 & 0.0135 & -6862 \\
$50423.36179^{\mathrm{a}}$ & 0.0009 & 0.0018 & -5297 \\
$50748.74373^{\mathrm{a}}$ & 0.0047 & -0.0540 & -3600 \\
$50748.95188^{\mathrm{a}}$ & 0.0063 & 0.0316 & -3599 \\
53612.47423 & 0.0003 & -0.0184 & 11335 \\
54055.59920 & 0.0014 & -0.0162 & 13646 \\
54115.23676 & 0.0006 & 0.0078 & 13957 \\
54126.35826 & 0.0007 & 0.0091 & 14015 \\
54129.62484 & 0.0008 & 0.0451 & 14032 \\
54174.27501 & 0.0072 & -0.0937 & 14265 \\
\hline
\end{tabular}

a Schwarz et al. (1998)

The orbital period of AI Tri has not been known accurately enough to extrapolate the photometric ephemeris given by Schwarz et al. (1998) to the epochs of the new observations. Using timings of six new $V$-band minima mapped between August 2005 and March 2007 and the data published by Schwarz et al. (1998), we have updated their ephemeris of the system. The heliocentric minima, listed in Table 5.2, are determined by Gaussian fits to the light curves excluding the sharp dips. By means of a least-square method, the inverse square sum of observed minus calculated minimum times $(O-C)^{-2}$ is minimized for narrowly spaced trial periods within a $10 \sigma$ range around the value of Schwarz et al. (1998). We derive the improved $V$-band ephemeris

$$
\operatorname{HJD}\left(T_{\min }\right)=2451439.0400(12)+0.19174568(10) \cdot E
$$

and use them throughout this work for folding the data with the photometric phase. 


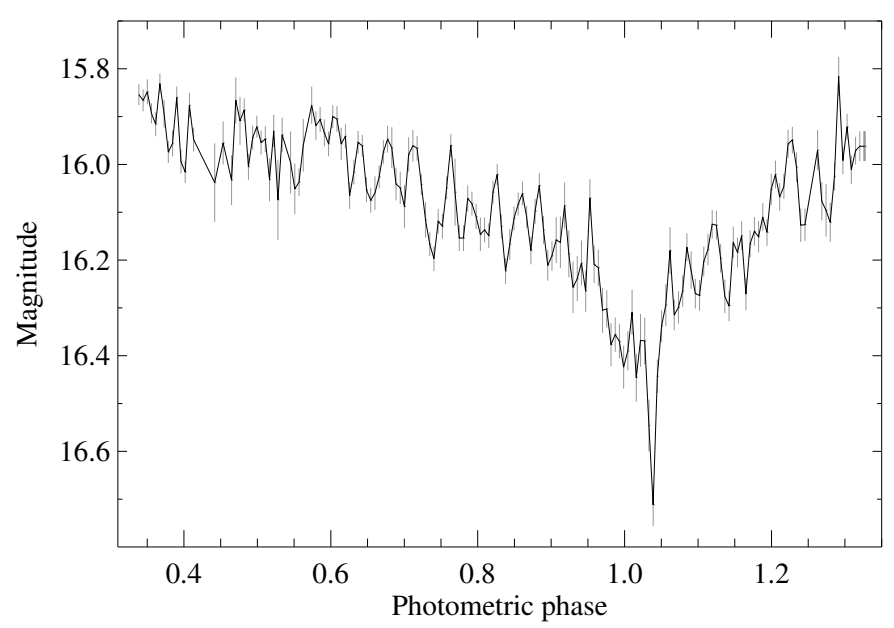

Figure 5.6: The high-resolution $V$-band light curve of AI Tri acquired on January 25, 2007 at the $70 \mathrm{~cm}$ telescope of the Astrophysical Institute Potsdam, ruled by flickering substructure on a time scale of several minutes. Cycle time is $100 \mathrm{~s}$.

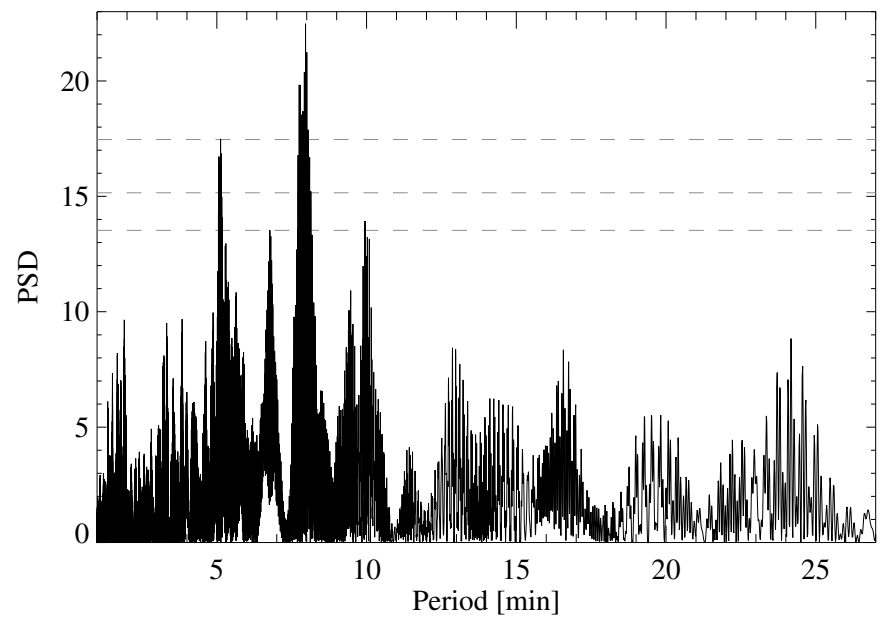

Figure 5.7: Periodogram based on the highest time resolved $V$-band data of January 2007 with the $95 \%, 99 \%$, and $99.9 \%$ significance levels in grey. The substructure is due to the daily alias pattern. 
The optical light curves are highly variable with strong and potentially recurring flickering, which Schwarz et al. (1998) describe as possible quasi-periodic oscillations (QPOs) at frequencies between $6.5 \mathrm{~min}$ and $14 \mathrm{~min}$. We examine three highly resolved light curves with cycle times below $100 \mathrm{~s}$ and proper signal-to-noise, obtained between January 25 and 30, 2007, to investigate this feature. In these light curves, substructure on a time scale of several minutes is clearly identifiable, as demonstrated for instance in Figure 5.6. After subtracting a strongly smoothed light curve to get rid of the dominating $4.6 \mathrm{hrs}$ cycle, a period search was performed according to the Lomb-Scargle analysis of unevenly spaced data (Lomb 1976; Scargle 1982). The periodogram shows the typical broad maxima, which could be associated to quasi-periodic flickering between $5 \mathrm{~min}$ and $10 \mathrm{~min}$, but may also arise from a superposition of different patterns (Figure 5.7). At two periods around $8 \mathrm{~min}$ and $5 \mathrm{~min}$, the power spectrum reaches the $99.9 \%$ confidence level according to Scargle (1982), so the features may be considered as real.

\subsubsection{Irregular mode on August 17, 2005}

The optical light curve of AI Tri obtained at the Potsdam $70 \mathrm{~cm}$ telescope on August 17, 2005 (Figure 5.8d) differs markedly from the majority of those taken between August 1993 and October 1997 (Schwarz et al. 1998) and between August 2005 and March 2007 (this work) at different sites. At a magnitude around $V=16^{\mathrm{m}} 0$ close to high state of accretion, its photometric minimum appears to be shifted by 0.2 in phase, the shape of the light curve is more asymmetric, and its amplitude larger than in normal states. In consequence, we have not included this peculiar light curve in the determination of the new ephemeris in Section 5.2.3. While the narrow minimum dip discovered in the $V$-band light curves from November 2006 on is missing, striking variability marks the lightcurve structure. The following $V$-band observation on August 29, 2005 fits in the prevailing shape again. Schwarz et al. (1998) describe a comparable phase shift with an altered light curve shape for their $R V$ photometry of October and November 1992 as 'irregular mode'. They point out changes in the accretion region, particular its shape, size, or location, as possible explanations for the feature.

The irregular mode is found in close proximity to the curtailed XMM-Newton observation on August 15, 2005 (Figure 5.8a-c). The EPIC count rate in the soft energy band with pronounced flickering is by a factor of about eight higher than one week later, whereas only little hard X-ray emission was registered 


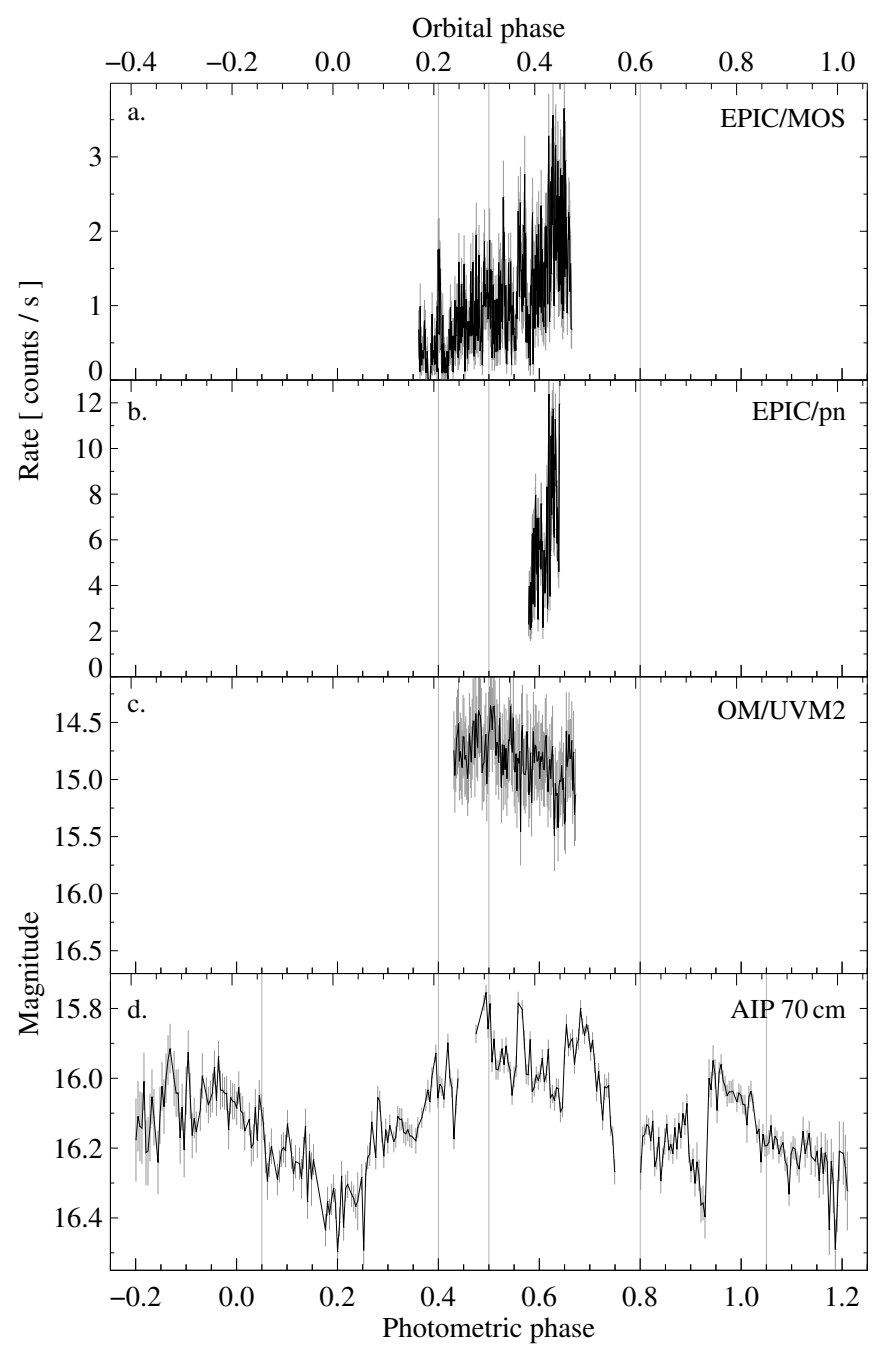

Figure 5.8: Multi-wave band light curves of AI Tri, obtained with XMM-Newton on August 15, 2005 and at the Potsdam $70 \mathrm{~cm}$ telescope on August 17, 2005 ( $V$-band data shown in the lowest panel). The X-ray light curves are binned to $10 \mathrm{~s}$ intervals, the ultraviolet to $30 \mathrm{~s}$ respectively. The grey lines mark the phases of high and low soft X-ray flux as stated for the $20 \mathrm{ksec}$ XMM-Newton exposure. 
during the short integration times of 1.1 to $5 \mathrm{ksec}$, not exceeding the low level of hard X-rays detected on August 22, 2005. The associated hardness ratios stay almost constant at about -0.87 , even during the $\varphi_{\text {phot }}=0.4-0.5$ interval, where they increase to zero in the August 22 data. The ultraviolet light curve resides at similar magnitudes as on August 22. Due to the insufficient coverage of the orbital period, the appearance of the soft X-ray minimum cannot be revealed in the August 15 data.

\subsection{X-ray spectroscopy}

The background subtracted X-ray spectra of AI Tri, obtained with the EPIC/pn, MOS1, and MOS2 detectors, have been analyzed by means of multi-component models in XSPEC v12.5 (Arnaud 1996; Dorman et al. 2003). The contribution of the white dwarf has been approximated by absorbed black bodies, mainly forming the soft spectral range below $0.5 \mathrm{keV}$ and originating in the accretion heated stellar surface around the base of the accretion column. To describe the interstellar absorption, the TBNEW model is employed with the abundances of Wilms et al. (2000) and the cross-sections following Verner \& Ferland (1996) and Verner et al. (1996). For an appropriate fit to the diffuse hot plasma in the post-shock flow, we have compared a number of different emission models (Table 5.3). This component requires the main effort as the models cannot accurately reflect the complicated and widely unknown temperature distribution in the (post-)shock region. Approximations like cooling flow spectra and multi-temperature plasma models are described in Chapter 3. Furthermore, potentially remaining traces of proton flares could influence the hard X-ray spectrum above 2 to $3 \mathrm{keV}$, where the flaring signal rises while the source count rate decreases simultaneously. MEKAL emission models (cf. Section 3.1.2) provide spectra that include continuum and element lines from a hot, optically thin plasma. The plasma component in the AI Tri spectra requires an additional absorption term to account for the material surrounding the emission region in the accretion column. We use the solar metal abundance ratios of Grevesse et al. (2007) in the MEKAL models and the partial covering PCFABS for the intrinsic absorption. EPIC/pn and MOS data are fitted simultaneously, since an individual treatment of the instruments does not raise the fit accuracy significantly, as F-statistic tests also exhibit.

A single black body gives a first approximation to the soft component, resulting in a mean temperature of $k T_{\mathrm{bb}}=39.5_{-2.5}^{+2.7} \mathrm{eV}$ and an interstellar ab- 


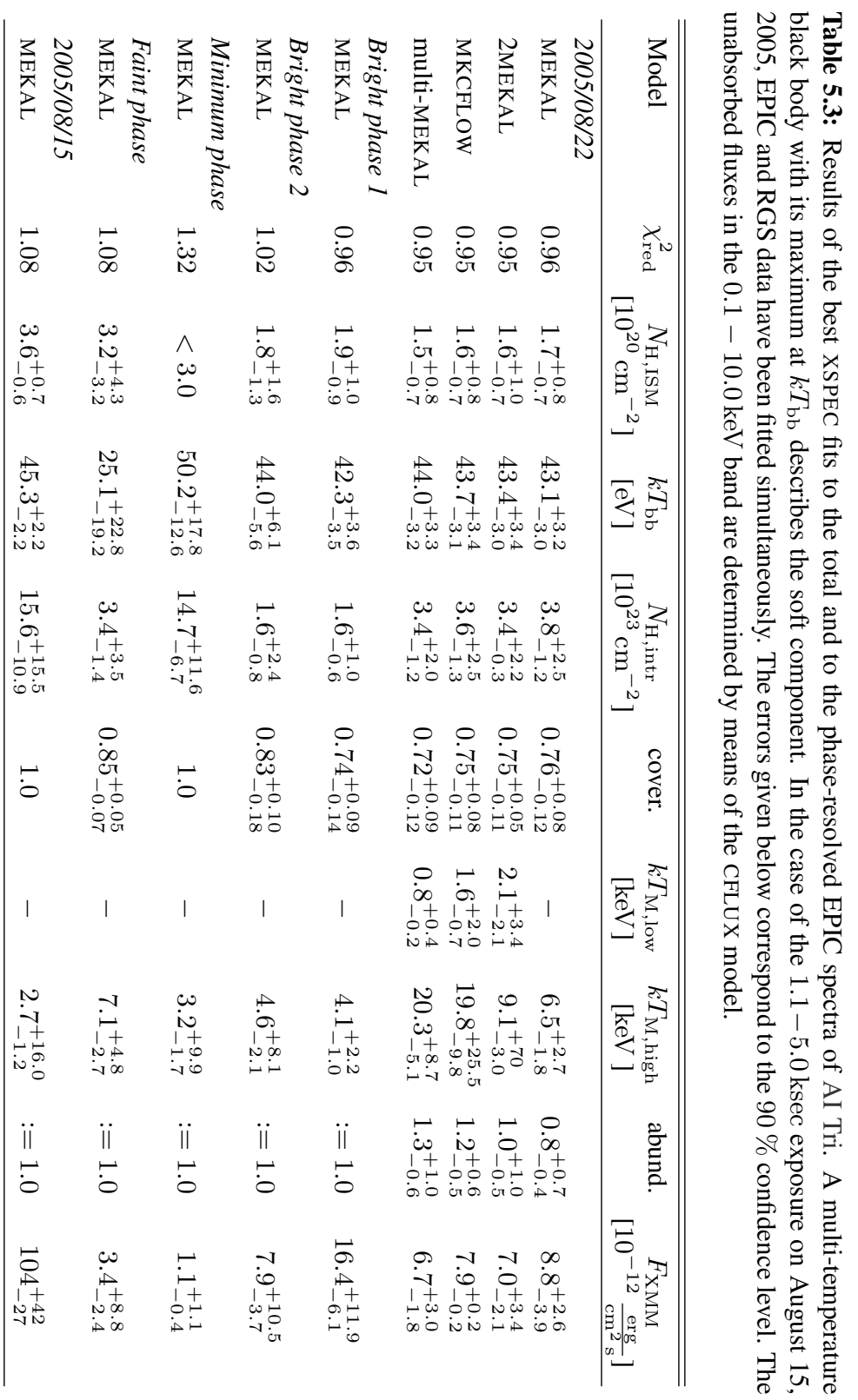


sorption of $N_{\mathrm{H}, \mathrm{ISM}}=1.4_{-0.7}^{+0.8} \cdot 10^{20} \mathrm{~cm}^{-2}$. As an alternative to the single black body model, we have fitted multi-temperature black bodies to the soft energy band as described in Section 3.2. With slightly enhanced fit statistics in the $0.15-0.5 \mathrm{keV}$ range (see Figure 5.9), the multi black body model at temperatures between $k T_{\mathrm{bb}, \text { low }}:=1 \mathrm{eV}$ and $k T_{\mathrm{bb}, \text { high }}=43.1_{-3.0}^{+3.2} \mathrm{eV}$ yields a higher flux towards ultraviolet wavelengths and comes along with a marginally higher column absorption of $N_{\mathrm{H}, \mathrm{ISM}}=1.7_{-0.7}^{+0.8} \cdot 10^{20} \mathrm{~cm}^{-2}$. The absorption given by TBNEW is thus on the same order as the galactic $N_{\mathrm{H}}$ of up to $5 \cdot 10^{20} \mathrm{~cm}^{-2}$ towards AI Tri listed by Kalberla et al. (2005) and Dickey \& Lockman (1990).

The accretion column spectrum is fitted well by MEKAL plasma emission at a mean temperature of $k T_{\text {MEKAL }}=6.5_{-1.8}^{+2.7} \mathrm{keV}$ and an element abundance of $0.8_{-0.4}^{+0.7}$ times the solar. Larger residuals remain around $1 \mathrm{keV}$ and between 3 and $5 \mathrm{keV}$. The multi-temperature plasma models, more precisely describing the emission lines, further improve the fit accuracy (Figure 5.10), as for example the preliminary CEMEKL model presented by Traulsen et al. (2007). The best convergence is achieved when using a multi-MEKAL plasma spectrum with the temperature structure following the models of Fischer \& Beuermann (2001). At a magnetic field strength of $B=40 \mathrm{MG}$ and an averaged mass flow density of $\dot{m}=0.1 \mathrm{~g} \mathrm{~cm}^{-2} \mathrm{~s}^{-1}$, it results in a reduced $\chi_{\text {red }}^{2}=0.96$ at $312 \mathrm{de}-$ grees of freedom, plasma temperatures between $k T_{\mathrm{MEKAL} \text {, low }}=0.8_{-0.2}^{+0.4} \mathrm{keV}$ and $k T_{\text {MEKAL,high }}=20.3_{-5.1}^{+8.1} \mathrm{keV}$, and $1.3_{-0.6}^{+1.0}$ times the solar metal abundance (Figure 5.11). The comparably low mean plasma temperature primarily is owing to the employment of the partial covering absorber with $N_{\mathrm{H} \text {,intr }}=$ $3.8_{-1.2}^{+2.5} \cdot 10^{23} \mathrm{~cm}^{-2}$.

Neither of the tested plasma models provides a finally satisfying description of the spectral slope at energies above $4 \mathrm{keV}$ (Figure 5.10). The deviations may stem from still unresolved emission lines, requiring a modified temperature structure or metal abundance. Alternatively, reflection of the continuum from the white-dwarf surface may harden the spectrum for energies above several keV, as Done et al. (1995) expound for the GINGA spectra of EF Eri. The residuals around $1 \mathrm{keV}$ can indicate photoionization effects. An additional warm absorber model component like ABSORI or XSTAR (Section 3.1.4) better accounts for the spectral shape, but introduces too many free parameters.

In addition to the EPIC spectra, we have converted the ultraviolet data into spectral information. The total OM/UVM2 flux in the wavelength range between $2050 \AA$ and $2450 \AA$ calculates from the corrected count rate using the conversion factor from white dwarf standards, where a count rate of $1 \mathrm{cts} \mathrm{s}^{-1}$ is equivalent to $F=2.2 \cdot 10^{-15} \mathrm{erg} \mathrm{cm}^{-2} \mathrm{~s}^{-1} \AA^{-1}$. Figure 5.12 shows the addi- 


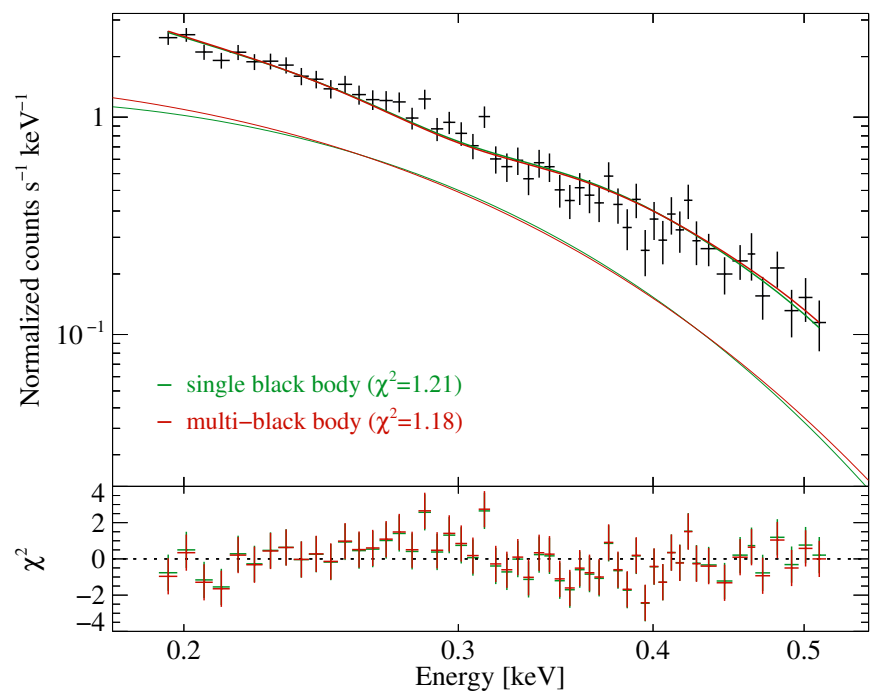

Figure 5.9: Comparison of unabsorbed single and multi-temperature black body models together with the soft part of the orbital mean EPIC/pn spectrum of AI Tri.

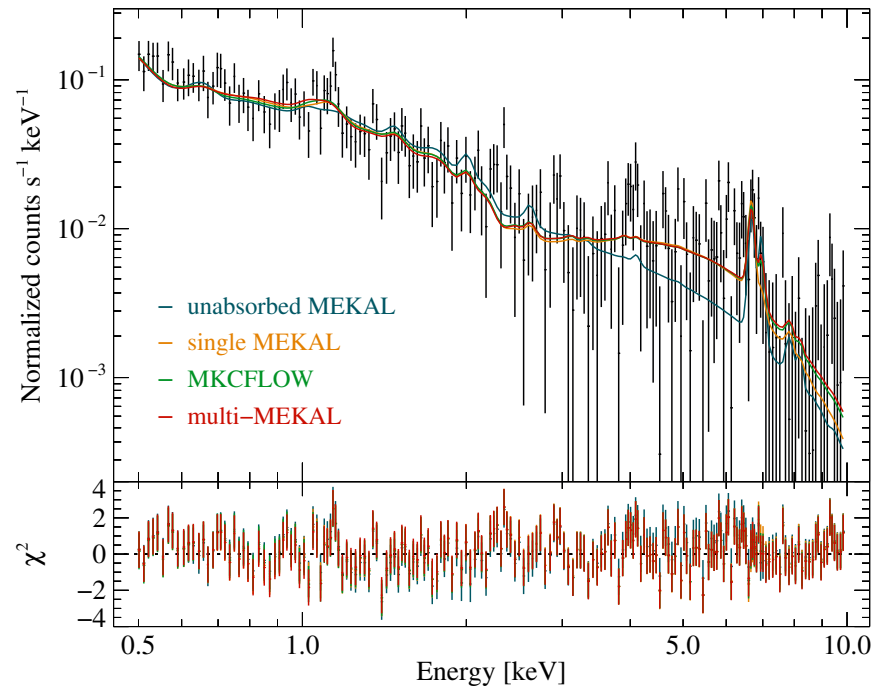

Figure 5.10: Single and multi-temperature plasma models together with the EPIC/pn spectrum at energies above $0.5 \mathrm{keV}$, binned to at least $20 \mathrm{cts} / \mathrm{bin}$. 
tional spectral point for the Optical Monitor and the EPIC/pn spectra together with the best-fit black body plus (multi-)MEKAL models for both the exposures on August 15 and 22, 2005. On August 22, the flux measured in the OM/UVM2 filter exceeds the extrapolated flux of the black body components by a factor of several hundred. Among the effects that may account for the discrepancy, two are decisive: The accretion stream gains in influence in the ultraviolet and particularly in the optical range, and the emitting surface area is supposed to be considerably larger in the ultraviolet regime. Moreover, the contribution of cyclotron emission to the UV flux is not included in the XSPEC model. The UV data point therefore has to be considered as an upper limit, which is not violated by the spectral components required to model the observed X-ray flux.

\subsubsection{Phase-resolved modeling}

The $20 \mathrm{ksec}$ XMM-Newton exposure has been divided into four phase intervals, characterized by the changing amount of soft X-ray emission and the associated hardness ratio (cf. Figure 5.3). Source and background spectra have been extracted and modeled for each of these intervals separately. With respect to the number of counts being lower than in the complete data set, we have used absorbed multi black body plus single MEKAL models, fixing the element abundances to the solar distribution.

Two broad maxima in the soft X-ray emission during $\varphi_{\text {phot }}=0.05-0.40$ and $\varphi_{\text {phot }}=0.50-0.80$ with hardness ratios around -0.7 govern the XMMNewton X-ray light curves. Consequently, the black body luminosity and the integrated observed X-ray flux exceed the averaged values from the best fits to the total spectrum. The hydrogen absorption as well as the black body and MEKAL temperatures agree remarkably well during the two bright phases. The intrinsic absorption drops to values around $N_{\mathrm{H} \text {,intr }}=1.6 \cdot 10^{23} \mathrm{~cm}^{-2}$, less than half the orbital mean. The spectral fit to the hard component of the second bright phase is affected with larger uncertainties in the mean temperature of the accretion column, because the signal-to-noise is poorer than during the first one. On the whole, the two bright phase spectra may be considered as identical within the error bars.

During the faint phase $\left(\varphi_{\text {phot }}=0.80-1.05\right.$, excluding an intermittent soft flare before $\varphi_{\text {phot }}=1.0$ ), the soft X-ray flux has dropped to its minimum and is dominated by the hard component, maximizing the hardness ratio. The integrated X-ray flux and particularly the black body luminosity diminish, whereas 
the temperatures stay almost constant with respect to the large error bars. The low signal-to-noise and the high background signal during the faint phase cause the uncertainties. The intrinsic absorption is with $N_{\mathrm{H}, \mathrm{intr}}=3.4_{-1.4}^{+3.5} \cdot 10^{23} \mathrm{~cm}^{-2}$ comparable to the value averaged over the whole orbital cycle.

The fit to the soft minimum phase around $\varphi_{\text {phot }}=0.45$ also suffers from a low count rate, resulting in a poorer fit accuracy. While the black body flux diminishes, the intrinsic absorption increases rapidly to $N_{\mathrm{H} \text {,intr }}=1.5_{-0.7}^{+1.2}$. $10^{24} \mathrm{~cm}^{-2}$, totally covering the emission region. This high column density comes along with an enhanced MEKAL norm, so that the plasma component has a larger part in the total X-ray flux within the XMM-Newton energy range.

\subsubsection{XMM-Newton data on August 15, 2005}

From the 1.1 to 5 ksec XMM-Newton observation, performed on August 15, 2005 close to the irregular $V$-band light curve described in Section 5.2.4, EPIC and RGS first order spectra have been extracted and fitted simultaneously (Figure 5.13). The EPIC/MOS and RGS data cover phases $\varphi_{\text {phot }}=0.35-0.65$ and EPIC/pn $\varphi_{\text {phot }}=0.58-0.65$ respectively, which is equivalent to the soft minimum phase and about the first half of the second bright phase in the $20 \mathrm{ksec}$ exposure on August 22, 2005 (Figure 5.8). Since most of the EPIC and RGS signal are detected at energies below $1 \mathrm{keV}$ and the hard X-ray component has almost vanished, we choose an absorbed multi black body model following Section 3.2 plus a single MEKAL with a fixed solar element abundance. With $k T_{\mathrm{bb}, \text { high }}=45.3_{-2.2}^{+2.2} \mathrm{eV}$ and $N_{\mathrm{H}, \mathrm{ISM}}=3.6_{-0.6}^{+0.7} \cdot 10^{20} \mathrm{~cm}^{-2}$, the black body parameters differ only marginally from the best fit to the $20 \mathrm{ksec}$ exposure on August 22, 2005. The flux measured in the OM/UVM2 filter is similar in both the observations. While it exceeds the extrapolated flux of the black body components on August 22, 2005, indicating that a major fraction of the UV emission originates in a different spectral component, the model with a lower limit of $k T_{\text {low }}=1 \mathrm{eV}(11600 \mathrm{~K})$ matches the ultraviolet data in the 2005 August 15 exposure (Figure 5.12). The hard X-ray flux contribution is considerably lower than on August 22, 2005, resulting in a MEKAL temperature of $k T_{\text {MEKAL }}=2.7_{-1.2}^{+16.0} \mathrm{keV}$. A high intrinsic absorption of $N_{\mathrm{H} \text {,intr }}=1.6_{-1.1}^{+1.6} \cdot 10^{24} \mathrm{~cm}^{-2}$ compensates for the steeper spectral shape. The source flux estimated from the XSPEC fits was at least by a factor of five higher than during the $20 \mathrm{ksec}$ exposure obtained one week later. This significant increase in the flux mainly arises from the dominant soft X-ray component and a larger emitting surface area. 


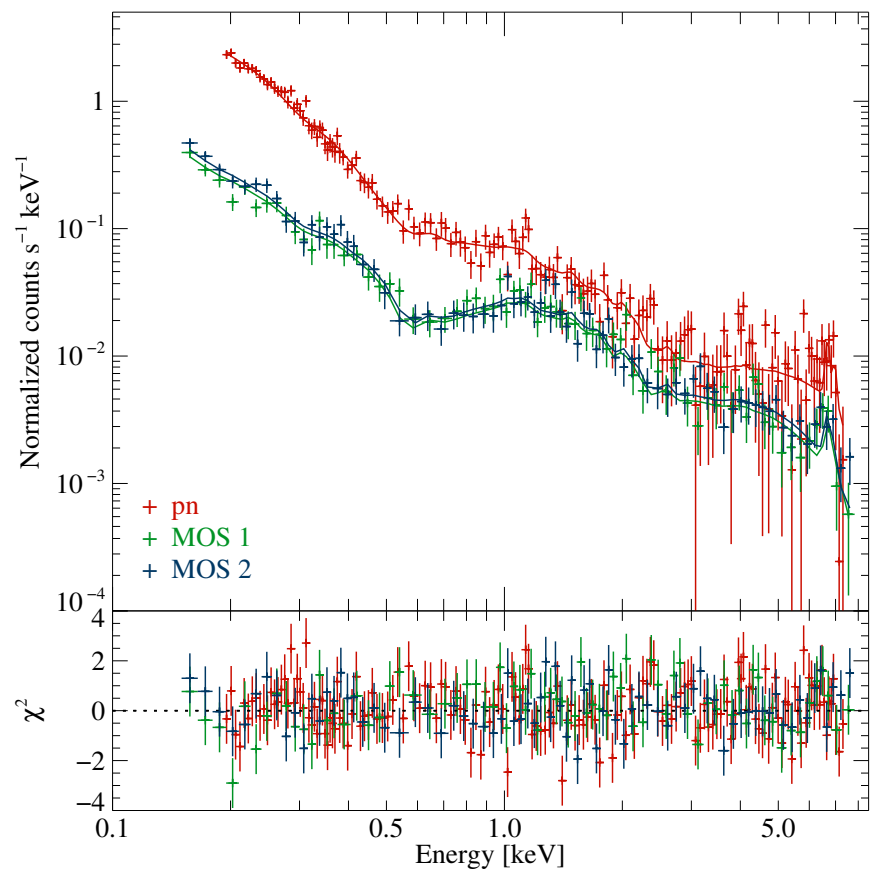

Figure 5.11: EPIC spectra extracted from the $20 \mathrm{ksec}$ exposure of AI Tri. The model consists of mildly absorbed black bodies at $N_{\mathrm{H}, \mathrm{ISM}}=1.5_{-0.7}^{+0.8} \cdot 10^{20} \mathrm{~cm}^{-2}$ and temperatures between $k T_{\mathrm{bb}, \text { low }}:=1 \mathrm{eV}$ and $k T_{\mathrm{bb} \text {, high }}=44.0_{-3.2}^{+3.3} \mathrm{eV}$, combined with multiMEKAL plasma emission between $k T_{\text {MEKAL, low }}=0.8_{-0.2}^{+0.4} \mathrm{keV}$ and $k T_{\text {MEKAL, high }}=$ $20.3_{-5.1}^{+8.7} \mathrm{keV}$. The averaged element abundance is $1.3_{-0.6}^{+1.0}$ times the solar.

\subsection{Discussion}

\subsubsection{The flaring structure of the light curves}

The light curves of polars in different wavelength regimes are linked to the individual components of the system, radiating at different energies. The optical light curves, stamped by emission from all the three components accretion stream, white dwarf, and secondary, are of complex nature. Since the optical light curves of AI Tri show no evidence of an eclipse by the secondary M star, the origin of their main modulation remains uncertain and could be due to 


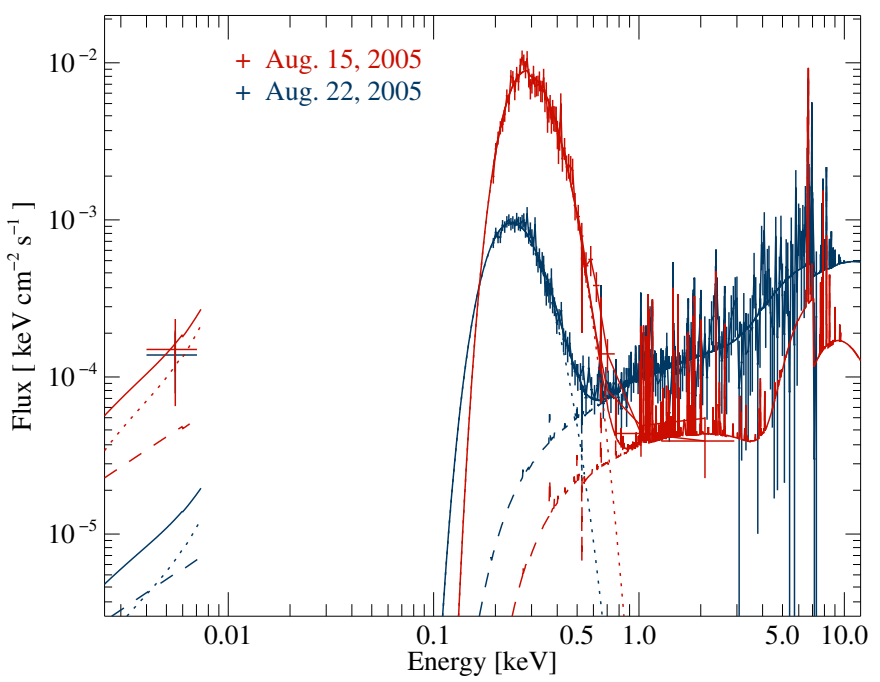

Figure 5.12: $\mathrm{EPIC} / \mathrm{pn}$ spectra from both the XMM-Newton pointings on August 15 and 22, 2005 together with the corresponding OM/UVM2 spectral points (left). Overplotted are the best-fit models (solid lines) as described in the text and the individual components absorbed black body (dotted) and MEKAL (dashed) for each exposure.

cyclotron beaming as pointed out by Schwarz et al. (1998).

The striking variability characterizing the X-ray as well as the optical light curves most likely originates in an inhomogeneous structure of the accretion stream. Kuijpers \& Pringle (1982) and Frank et al. (1988) ascribe this to the formation of lumpy dense blobs or filaments under the magnetic pressure in the system. These blobs excite rapid variations in the X-ray flux, when they impinge on the white-dwarf atmosphere, are thermalized, and release their energy in the form of additional soft X-ray and / or UV radiation. Individual accretion events cannot be identified in the time-resolved X-ray light curves of AI Tri, though their shape is probably formed by multiple superposition of emission from blobby events (cf. Figure 5.5). Single events, Schwarz et al. (2005) for example identify in the purely 'blobby' accreting polar V1309 Ori.

Substructure on time scales of several minutes appears in the optical light curves of AI Tri. Schwarz et al. (1998) describe quasi-periodic oscillations (QPOs) at frequencies of 6.5 - $7 \mathrm{~min}$ and 13.5 - $14 \mathrm{~min}$ in their multi-wave band photometry of AI Tri, for the first time simultaneously seen at two fre- 


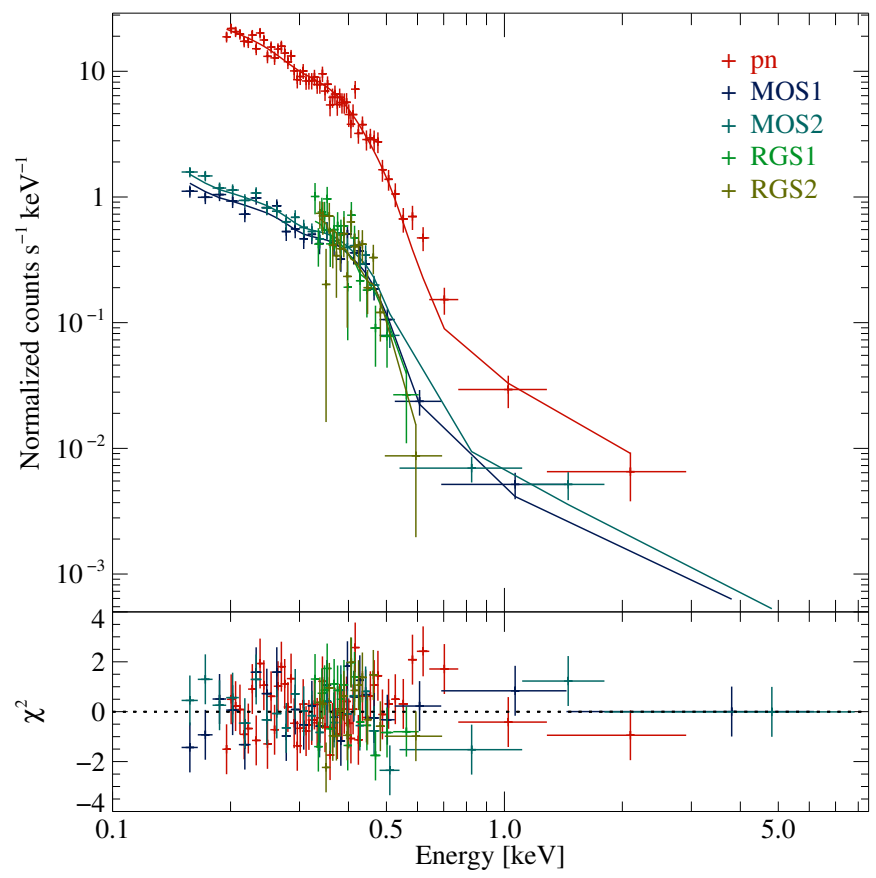

Figure 5.13: Simultaneous multi-temperature black body plus MEKAL fit to the EPIC and RGS spectra on August 15, 2005 with integration times between $1.1 \mathrm{ksec}$ and $5 \mathrm{ksec}$. Only little flux has been registered in the hard energy band at $0.5-10.0 \mathrm{keV}$, and it shows a steeper shape than in the $20 \mathrm{ksec}$ exposure obtained one week later.

quency multiples. A Lomb-Scargle period analysis of the high time-resolution $V$-band data of January 2007 reveals possible QPO periods of $8 \mathrm{~min}$ and - less significant - of $5 \mathrm{~min}$, but no evidence of a $14 \mathrm{~min}$ periodicity in this shortlived feature (Section 5.2.3). The smaller peaks around $6.8 \mathrm{~min}$ and $10 \mathrm{~min}$ hardly reach the $90 \%$ confidence level during our observations and therefore might not be considered as significant.

Furthermore, the optical light curves are superposed by additional dips migrating in phase. The recurrent sharp dip that appears during the light curve minimum at photometric phase zero at least from November 2006 on, possibly is correlated with the diversion of the accretion stream out of the orbital plane near the stagnation region. A quasi-simultaneous dip in the UV flux, a broad 
$\mathrm{X}$-ray minimum, and a sharp increase in the hardness ratio up to $\mathrm{HR}=1.0$, supporting the idea of stream absorption, accompany the minimum dip at the corresponding phase in the 2005 August 22 XMM-Newton observation. In the photo-polarimetric data of November 1998 presented by Katajainen et al. (2001), a linear polarization peak arises around $\varphi_{\text {phot }}=0.0$. The existence of a minimum dip in the regular $V$-band light curve on August 29, 2005, taken during intermediate high state of accretion at a mean brightness of $V \sim 17^{\mathrm{m}} 5$, cannot be convincingly stated with respect to the low flux. Additional singular dips seem not to be permanent features. They could be interpreted as another sign for an inhomogeneous cross section of the accretion stream or might be caused by other geometrical effects. The occurrence of irregular optical light curves in October/November 1992 (Schwarz et al. 1998) and on August 17, 2005, and the long-term evolution in the shapes of the soft X-ray light curves (Section 5.4.2) emphasize the diversity in the system's appearance.

\subsubsection{The nature of the soft $X$-ray minima}

Orbital ephemeris of the system have been determined by Schwarz et al. (1998) from long-slit spectroscopy. They define $\varphi_{\text {orb }}=0$ at the blue-to-red zero crossing of the radial velocities and identify it with the inferior conjunction of the secondary. Assuming a constant relation between orbital and photometric phase, we adopt their result $\varphi_{\text {phot }}-\varphi_{\text {orb }}=0.191 \pm 0.080$ to refer to orbital phase in the following.

The X-ray light curves obtained with ROSAT and XMM-Newton between January 1991 (Schwarz et al. 1998) and August 2005 (this work) show a variety of shapes, particularly in the soft component, and only little repeatability. Two minima imprint the XMM-Newton light curves shown in Figure 5.3 near $\varphi_{\text {phot }}=0.0\left(\varphi_{\text {orb }}=0.81\right)$ and $\varphi_{\text {phot }}=0.45\left(\varphi_{\text {orb }}=0.26\right)$, mainly pronounced in the soft X-ray flux and therefore most likely representing the orbital cycling of the bright accretion region and column. The ROSAT HRI light curves taken between January 15 and February 5, 1998 show similar shapes, but lack a definitive soft $X$-ray minimum around $\varphi_{\text {orb }}=0.26$. The former ROSAT data presented by Schwarz et al. (1998) differ from the more recent observations. A single distinct bright phase during $\varphi_{\text {phot }}=0.9-1.3\left(\varphi_{\text {orb }}=0.7-1.1\right)$ dominates the ROSAT PSPC light curves of July / August 1993, accompanied by a low hardness ratio close to HR $=-1$. In the ROSAT HRI data of August 1995 , a high count rate after $\varphi_{\text {phot }}=0.5\left(\varphi_{\text {orb }}=0.3\right)$ conforms with the second bright phase of the XMM-Newton observations. Many of the observed 


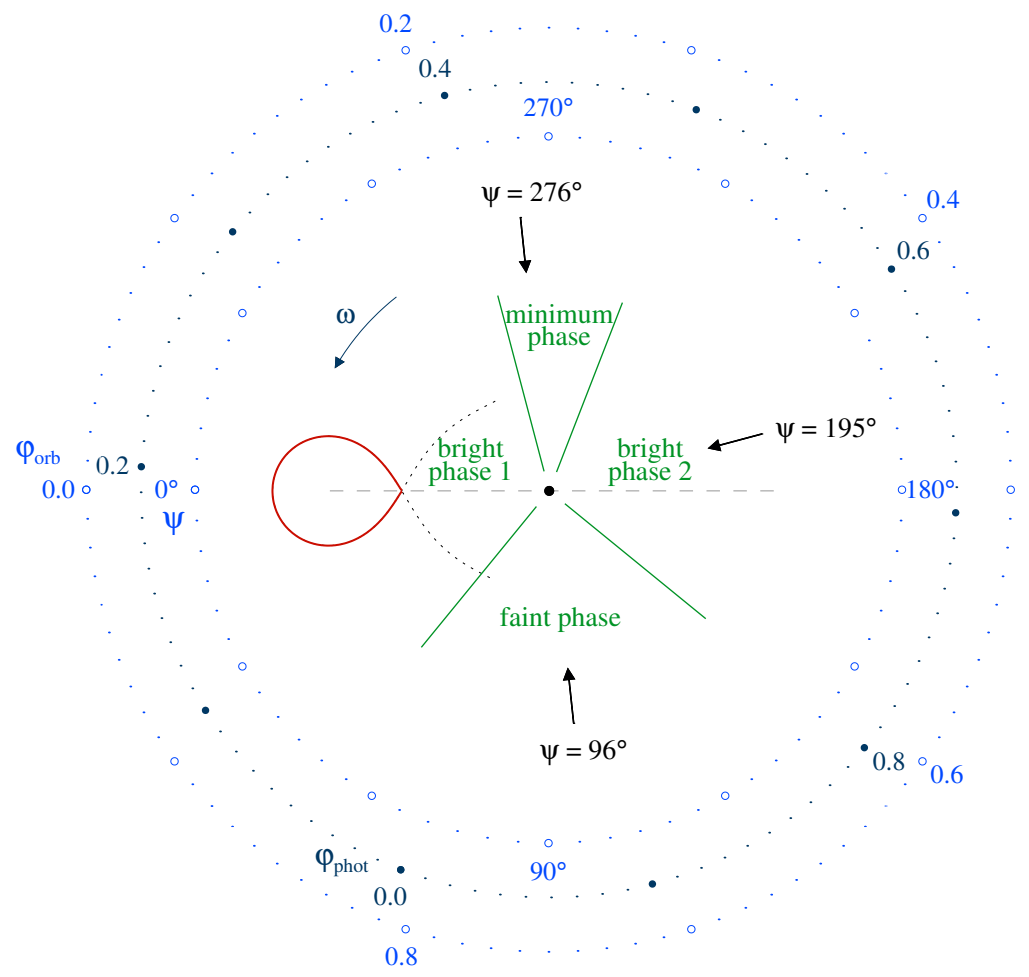

Figure 5.14: Sketch of the binary geometry on the basis of the spectroscopic phasing derived by Schwarz et al. (1998) with the Roche lobes calculated for a mass ratio of $q=\mathrm{M}_{\mathrm{WD}} / \mathrm{M}_{2}=1.5 . \varphi_{\text {phot }}$ and $\varphi_{\text {orb }}$ mark the photometric and the spectroscopic phase respectively; $\psi$ is the longitude on the white dwarf (angular distance to the connecting line between primary and secondary). The locations of the individual phase intervals are indicated to illustrate the formation of the most prominent light curve features.

features change in the long term, including the soft X-ray minimum around $\varphi_{\text {orb }}=0.26$, which most prominently shows up in the XMM-Newton data on August 22, 2005. A pronounced faint phase before or around $\varphi_{\text {orb }}=0.8$ $\left(\varphi_{\text {phot }}=0.0\right)$, though, appears to be common to all the X-ray light curves and largely coincides with the UV and optical minima and with a pronounced peak 
in the linear polarization (Katajainen et al. 2001).

The minima in the X-ray light curves in principle may have their origin in a variety of mechanisms. These include an eclipse by the secondary, emission from two independent accreting poles, random mass transfer variations on time scales of several hours, a total or partial self-eclipse of the accretion region by the white dwarf or absorption in the accretion stream. Since both the X-ray minima around $\varphi_{\text {orb }}=0.26\left(\varphi_{\text {phot }}=0.45\right)$ and $\varphi_{\text {orb }}=0.81\left(\varphi_{\text {phot }}=0.0\right)$ occur far off the inferior conjunction, none of them can be explained with an occultation of the white dwarf by the secondary star. The optical light curves also show no evidence of eclipses.

The existence of two accreting poles, visible around phases $\varphi_{\text {orb }}=0.034$ and $\varphi_{\text {orb }}=0.459$ respectively, could give an alternative explanation for the two broad maxima in the XMM-Newton light curves. Katajainen et al. (2001) favor a two-pole accretion geometry on the basis of their polarimetry data. If the accreting poles pass the line of sight at the center of the bright phases, they are located at longitudes of $\psi=195^{\circ}$ and $\psi=348^{\circ}$ on the white dwarf. This were a rather peculiar accretion geometry where the accretion stream was forced to pass almost the whole accretor to reach the second pole (cf. Figure 5.14) or where a major part of the accreted material had to move against the direction of the binary rotation. Both scenarios involve an energetically very inefficient process. We thus consider one main bright pole, centered around $\varphi_{\text {orb }}=0.234$, as the dominant source of the bright soft X-ray emission. This still leads to an unusual longitude $\psi=276^{\circ}$ of the accretion region, but is more strongly supported by the phase-resolved spectral models (Section 5.3.1).

A self-eclipse when the accretion region passes behind the limb of the white dwarf is in accordance with the length of the major faint phase, whilst the dip phase interval with $\Delta \varphi=0.1$ is too short to be convincingly explained by this means. During $\varphi_{\text {orb }}=0.21-0.31$, the hardness ratio nears zero, whilst it temporarily reaches $\mathrm{HR}=1$ within the faint phase, where the hard X-ray flux is expected to vanish in the case of a self-eclipse. The remaining low hard $\mathrm{X}$-ray flux could originate in an extended emission region that is not totally eclipsed or in a potential second accretion pole only visible in the high energy range. With the inclination $i \sim 70^{\circ}$ of the system (Katajainen et al. 2001), a partial or total self-eclipse is plausible, if the accretion region is located at a colatitude larger than about $20^{\circ}$.

Stream absorption when the accretion column crosses the line of sight, obscuring large parts of the accretion region and causing dips with a typical duration of $\Delta \varphi=0.1$ in the soft X-ray light curves, may also induce the soft 
X-ray minima. The decline in the X-radiation detected around $\varphi_{\text {orb }}=0.26$, which coincides with the maxima of the optical and the UV light curves, most persuasively arises from such a stream dip. The short ingress and egress times, the duration of the phase interval, and the hardness ratio close to zero are consistent with an eclipse of the accretion region by the stream. Figures 5.15 and 5.16 illustrate the geometry of a system with non-eclipsing component stars $\left(i=70^{\circ}\right)$ and with an occultation of the accretion region by the stream. The length of the faint phase around $\varphi_{\text {orb }}=0.81$, on the contrary, and the slow ingress do not match with the characteristics of a stream dip. If this interval though corresponded to stream absorption, the accretion region would be found at a longitude of $\psi=96^{\circ}$, consistent with the standard accretion geometry of polars (Cropper 1988). In consequence, the optical and the UV light curves surprisingly would be in anti-phase with the X-ray data. Osborne et al. (1987) describe such a scenario for QQ Vul, another long-period polar.

Mostly, stream absorption dips come along with an evident rise in the hydrogen column density. The temporary increase in the hardness ratio from -0.8 up to values around 0.0 (Figure 5.3) during the phase of the soft X-ray minimum confirms this characteristics. Under the assumption that the spectral parameters stay largely constant over the whole orbital cycle, as also indicated by the phase-resolved spectral fits (Section 5.3.1), the variations in the hardness ratio are directly connected to simultaneous changes of the column density, in particular to an increased absorption around $\varphi_{\text {orb }}=0.26$. The partial covering absorption terms in the phase-resolved black body plus MEKAL fits show the expected behavior, diminishing during the bright phases and markedly rising during the minimum phase, and thus corroborate the interpretation of the flux minimum around $\varphi_{\text {orb }}=0.26$ as stream absorption.

Figure 5.15: (following pages)

Example of a non-eclipsing polar that would produce a stream dip in the X-ray light curve. The system geometry and a closer view to the accreting white dwarf are illustrated for different orbital phases (not corresponding to the phasing of AI Tri).

$\varphi=0.15$ : The accretion region passes behind the white-dwarf limb. $\varphi=0.30$ : Faint phase. $\varphi=0.50$ : The accretion region reappears, and the bright light curve interval commences. $\varphi=0.70$ : First part of the bright phase. $\varphi=0.88$ : Stream eclipse, dip phase. $\varphi=1.00$ : Inferior conjunction, second part of the bright phase.

System parameters in this simulation are $i=70^{\circ}, P_{\mathrm{orb}}=4.6 \mathrm{hrs}, B_{\mathrm{WD}}=40 \mathrm{MG}$, $M_{\mathrm{WD}}=0.6 \mathrm{M}_{\odot}$, and $q=M_{1} / M_{2}=1.5$. The accretion pole is located at an azimuth of $\psi=95^{\circ}$ and a colatitude of $\beta=50^{\circ}$.

Source code by A. Staude, kindly provided by R. Schwarz, Potsdam. 

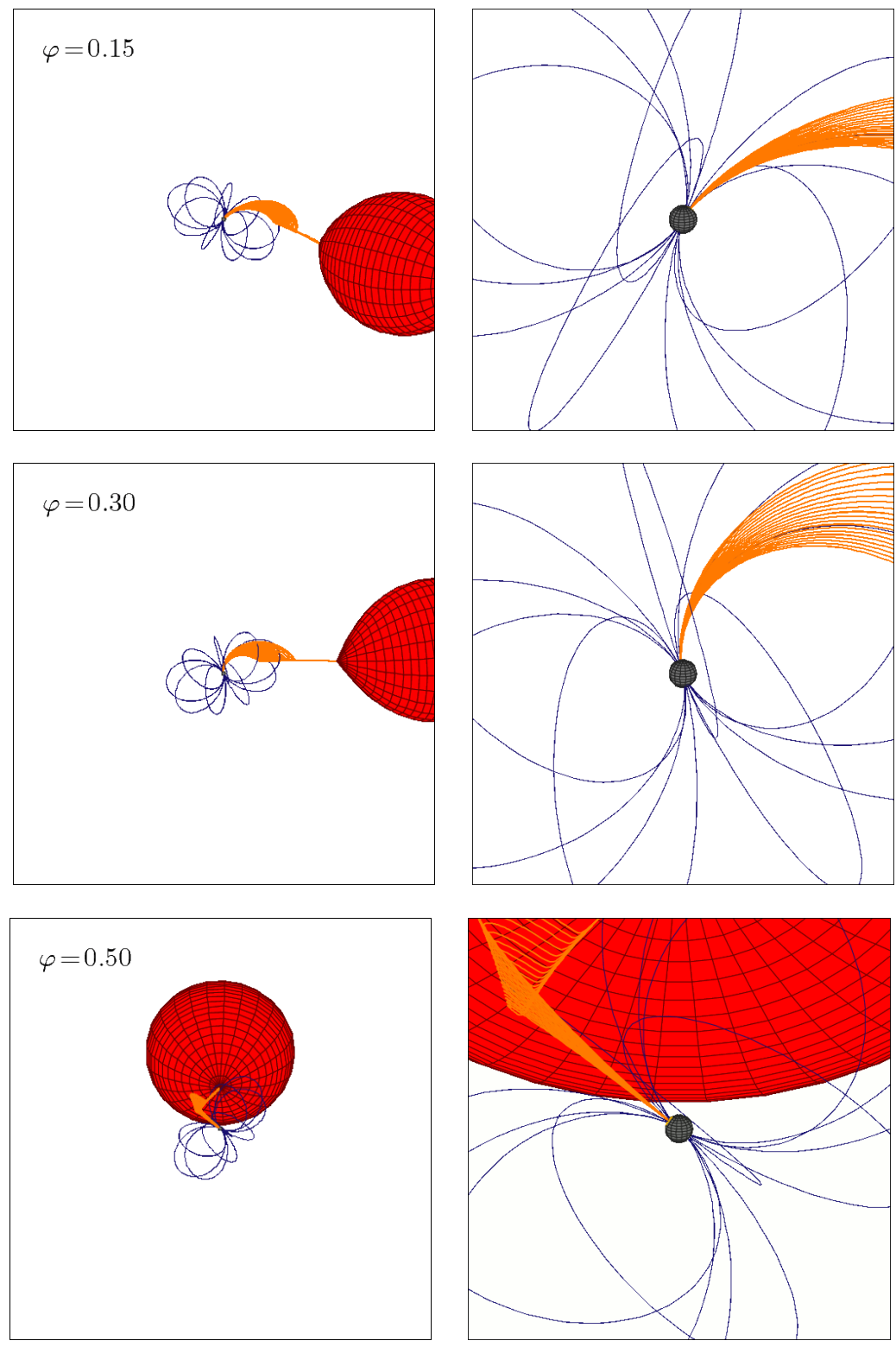

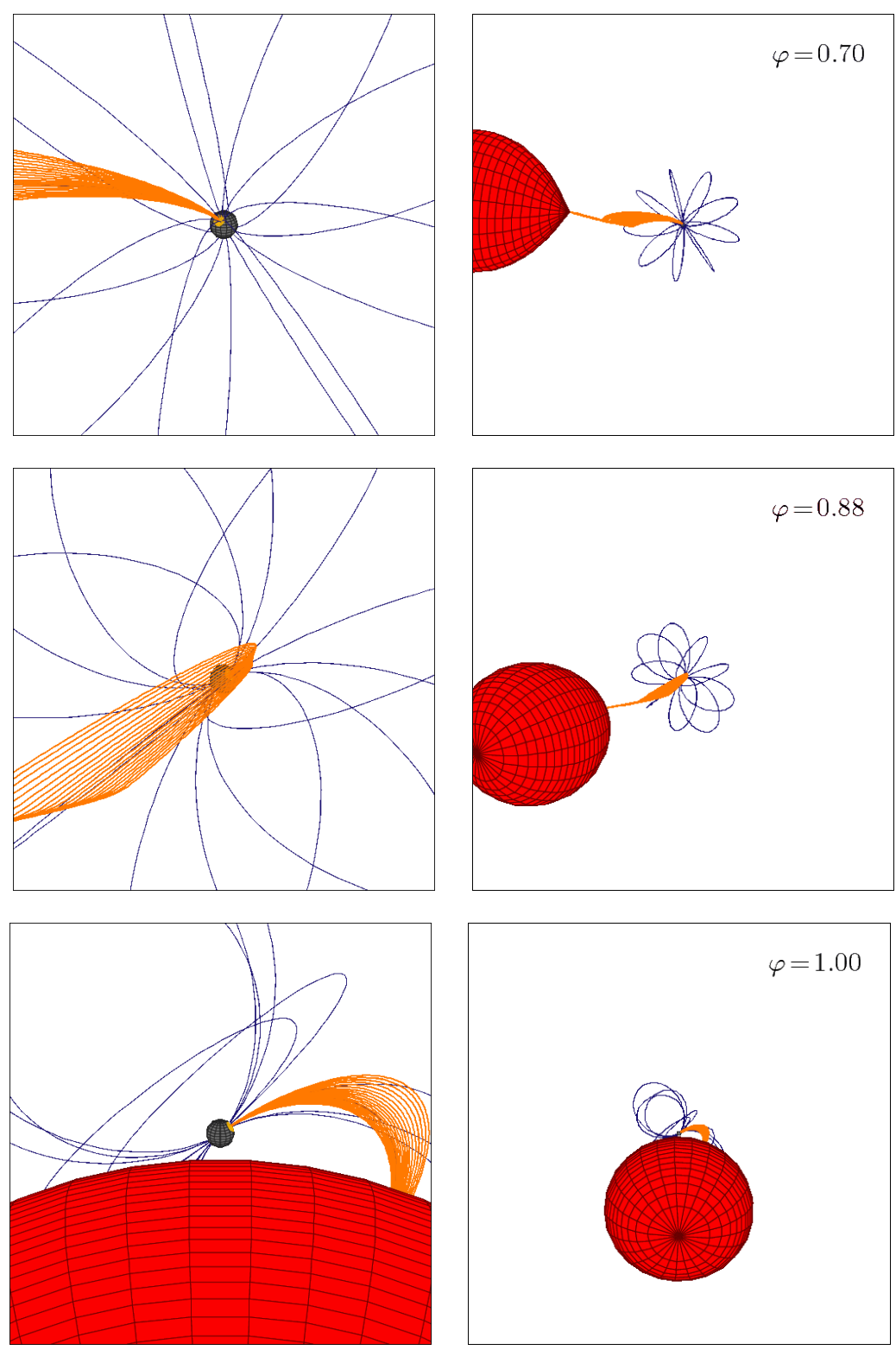


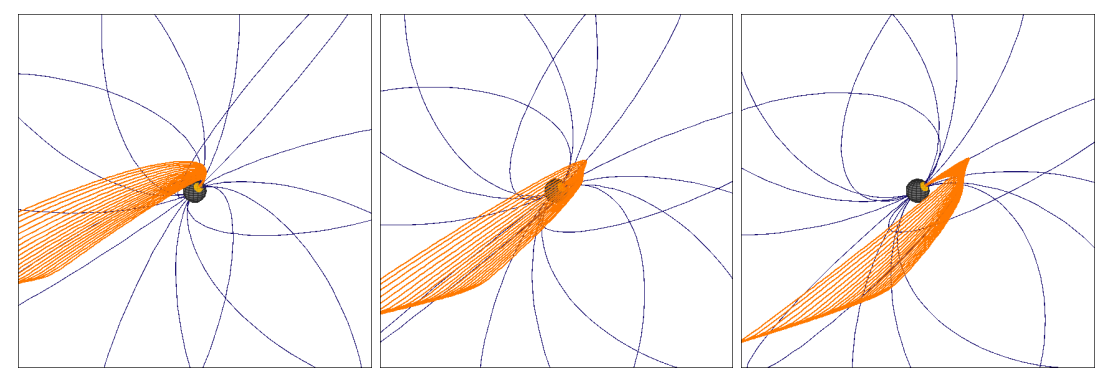

Figure 5.16: Illustration of the accretion stream crossing the line of sight and eclipsing the soft X-ray emission region on the white dwarf. System parameters as in Figure 5.15.

\subsubsection{Geometry of the system}

As shown in the previous Section, the most convincing accretion geometry of AI Tri comprises one main self-eclipsing accretion region which is visible from $\varphi_{\text {orb }}=0.86$ to $\varphi_{\text {orb }}=0.61$, interrupted by a possible stream absorption dip around $\varphi_{\text {orb }}=0.26$. Self-eclipses of the accretion region require a relatively high inclination of the system, which on the other hand is restricted by the lack of eclipses by the secondary. This is consistent with the conclusions of Schwarz et al. (1998) from optical high-resolution spectroscopy and of Katajainen et al. (2001) from their polarimetric study. The center of the bright phases at $\varphi_{\text {orb }}=0.234$, close to the absorption dip, corresponds to a longitude $\psi=276^{\circ}$ of the accretion region. The available data give no clue on the position of a potential second accreting pole on the white dwarf. Under the assumption of a phase shift of $\varphi_{\text {phot }}-\varphi_{\text {spec }}=0.191 \pm 0.080$ (Schwarz et al. 1998), this exceptional system geometry departs from the standard picture described for example by Cropper (1988). Mainly constrained by the synchronous rotation of the system, the azimuth is normally found between $\psi=0^{\circ}$ and $\psi=90^{\circ}$. A standard geometry might be preserved, in case the system rotated asynchronously with at least a small degree of asynchronism around $\left|P_{\text {orb }}-P_{\text {spin }}\right| / P_{\text {orb }}=2-5 \cdot 10^{-5}$. The resulting shift between photometric and spectroscopic phase at the time of the XMM-Newton observations would place the accretion region within the first quadrant, referring to the mid of the bright phase in the X-ray light curves. Schwarz et al. (1998) estimate an upper limit of $10^{-4}$ for the degree of synchronism from their phase-resolved spectroscopy and photometry. 
Puzzling questions arise from the XMM-Newton and archival ROSAT data: The emission during the second bright phase is lower than during the first one; the count rate changes distinctly between the different X-ray observations. An expanded accretion curtain along the ballistic accretion stream may reduce the flux between $\varphi_{\text {orb }}=0.3$ and $\varphi_{\text {orb }}=0.7$, as confirmed in the case of HU Aqr by Schwope et al. (2001). This accretion curtain thus would account for the slightly enhanced absorption in the spectral fits during the second bright phase. The asymmetric shape of the narrow emission line components in the optical spectra additionally supports this idea (Schwarz et al. 1998). By more or less effective shielding, the accretion curtain could induce the other strong variations detected in the X-ray light curves. Schwope et al. (2001) report similar effects for HU Aqr. Other possible reasons for the long-term evolution of the light curves are changes in size, structure, or location of the accretion region or switches between one-pole and two-pole accretion mechanisms, but there is no definite evidence of any of these effects found in the present 'regular mode' data.

In contrary, the irregular XMM-Newton and optical light curves on August 15 and 17, 2005 (Sections 5.2.4, 5.3.2) could belong to a change in the accretion geometry, as indicated by the high black body luminosity, the increased emitting surface area, and the shift of the optical light curve minimum by 0.2 in phase. This might correspondingly explain the irregular mode of the $V$-band light curves on October / November 1992 (Schwarz et al. 1998).

\subsubsection{On the soft-to-hard ratio}

Following the first identification of AI Tri as a soft X-ray source (Beuermann $\&$ Thomas 1993) and an approximate averaged hardness ratio HR $1=-0.96 \pm$ 0.03 in the $0.1-0.4 \mathrm{keV}$ against the $0.4-2.4 \mathrm{keV}$ bands listed in the RASS2 catalog (Voges et al. 1999), Schwarz et al. (1998) again stress the distinct soft $\mathrm{X}$-ray excess in their analysis of ROSAT data and determine hardness ratios HR 1 between 0.0 and -0.8 during the different orbital phases. With the two broad soft maxima dominating the XMM-Newton light curves, the soft X-ray excess in AI Tri is obvious. The averaged hardness ratio of the $2005 \mathrm{Au}-$ gust 22 XMM-Newton data, calculated in the ROSAT energy bands, lies with $\mathrm{HR} 1_{\text {bright }}=-0.63 \pm 0.03$ in a similar range during both the bright phases. Due to the larger number of hard counts within the spectral range covered by the XMM-Newton EPIC instruments, the mean hardness ratios in the EPIC bands during the bright phases are lower than the one given in the RASS2 survey, but 
Table 5.4: Hardness ratios and unabsorbed model fluxes in the $0.1-0.5 \mathrm{keV}$ and $0.5-$ $10.0 \mathrm{keV}$ energy bands for the phase-resolved AI Tri spectra and the best-fit models.

\begin{tabular}{lccc}
\hline \hline & HR & $\begin{array}{c}F_{\text {soft }} \\
{\left[10^{-12} \mathrm{erg} \mathrm{cm}^{-2} \mathrm{~s}^{-1}\right]}\end{array}$ \\
\hline $2005 / 08 / 22$ & $-0.43 \pm 0.09$ & $5.4_{-1.8}^{+3.3}$ & $1.2_{-0.1}^{+0.1}$ \\
Bright phase 1 & $-0.56 \pm 0.02$ & $14.9_{-5.9}^{+11.1}$ & $1.6_{-0.2}^{+0.2}$ \\
Bright phase 2 & $-0.53 \pm 0.05$ & $7.1_{-3.7}^{+13.0}$ & $0.8_{-0.2}^{+0.2}$ \\
Minimum phase & $-0.43 \pm 0.01$ & $0.6_{-0.2}^{+2.7}$ & $0.5_{-0.2}^{+0.5}$ \\
Faint phase & $+0.53 \pm 0.01$ & $2.4_{-2.3}^{+28.9}$ & $1.0_{-0.1}^{+0.2}$ \\
2005/08/15 & $-0.94 \pm 0.06$ & $104_{-28}^{+65}$ & $0.8_{-0.3}^{+0.3}$ \\
\hline
\end{tabular}

still clearly dominated by soft X-ray emission (Table 5.4). In the brighter 2005 August 15 data, $\mathrm{HR}_{\text {mean }}=-0.94 \pm 0.06$ extends to an equivalent level as the former ROSAT observations and is hardly influenced by counts above $2 \mathrm{keV}$.

Our best-fit multi-temperature black body plus MEKAL model to the 2005 August 22 XMM-Newton spectra yields the typical parameters of the highenergy distribution of CVs. Using the distance estimate of AI Tri (Schwarz et al. 1998) $d=620 \pm 100 \mathrm{pc}$, it results in an unabsorbed black body luminosity of $L_{\mathrm{bb}}=2.9_{-1.1}^{+1.9} \cdot 10^{32}(d / 620 \mathrm{pc})^{2} \mathrm{erg} \mathrm{s}^{-1}$ and an integrated X-ray flux $F_{\text {bol }}=1.3_{-0.6}^{+0.5} \cdot 10^{-11} \mathrm{erg} \mathrm{cm}^{-2} \mathrm{~s}^{-1}$. During the second bright phase, the total integrated flux reaches with $F_{\text {bright }, 2}=1.0_{-0.4}^{+2.0} \cdot 10^{-11} \mathrm{erg} \mathrm{cm}^{-2} \mathrm{~s}^{-1}$ less than half the flux of bright phase $1, F_{\text {bright, } 1}=2.4_{-0.8}^{+2.6} \cdot 10^{-11} \mathrm{erg} \mathrm{cm}^{-2} \mathrm{~s}^{-1}$, possibly lowered by absorption in an expanded accretion curtain (Section 5.4.3). The model fluxes in the soft and in the hard energy band $(0.1-0.5 \mathrm{keV}$ and $0.5-10.0 \mathrm{keV}$ respectively), shown in Table 5.4, reveal lower limits for the flux balance of $F_{\text {soft }} / F_{\text {hard }}=4.5_{-1.5}^{+2.8}$ and of $\left(F_{\text {soft }} / F_{\text {hard }}\right)_{\text {bright }, 1}=9.3_{-3.9}^{+7.0}$, $\left(F_{\text {soft }} / F_{\text {hard }}\right)_{\text {bright }, 2}=8.9_{-5.1}^{+16.4}$ during the bright phases. The flux in the soft $0.1-0.5 \mathrm{keV}$ band exceeds the hard flux during the whole orbital cycle (Table 5.4). The bolometric fluxes of the individual unabsorbed model components lead to lower ratios of $F_{\mathrm{bb}} / F_{\text {MEKAL }} \gtrsim 2.1_{-1.0}^{+1.9}$ and of $\left(F_{\mathrm{bb}} / F_{\text {MEKAL }}\right)_{\text {bright }, 1} \gtrsim$ $5.7_{-3.0}^{+6.1}$, as the multi-temperature MEKAL model, comprising components at temperatures down to $0.8 \mathrm{keV}$, significantly contributes to the flux in the soft energy band. In the short 2005 August 15 data, the total integrated unabsorbed flux $F_{\text {bol }}=1.6_{-0.5}^{+0.8} \cdot 10^{-10} \mathrm{erg} \mathrm{cm}^{-2} \mathrm{~s}^{-1}$ is notably governed by the black 
body component, while the model flux in the hard band stays at a low level, effecting a flux ratio of $F_{\text {soft }} / F_{\text {hard }}=130_{-60}^{+95}$. The black body flux, remarkably higher than in the 2005 August 22 observation, indicates an increase in the mass accretion rate by a factor of more than 100 . The enhanced mass accretion may induce a stronger temporal and spatial inhomogeneity of the accretion stream, forcing more material into dense 'blobs'. In consequence, the contribution of the hard X-ray emission will shrink compared to states of lower accretion rates, causing a distinct change in the spectral shape as seen in Figure 5.12. The pronounced flickering in the X-ray light curves agrees with a greater influence of blobby accretion.

Thus, a connection to a high magnetic field strength causing strong cyclotron emission to be the main cooling process as suggested by Beuermann \& Burwitz (1995) and to a blobby accretion scenario (Kuijpers \& Pringle 1982) may be plausible explanations for the considerable domination of the soft over the hard $\mathrm{X}$-ray component.

\subsection{Conclusions}

High variability on short time scales both in the optical and in the X-ray light curves are a sign for inhomogeneous, 'blobby' accretion, which may at least partly account for the distinct soft X-ray excess in AI Tri, consistently found in the archival ROSAT and the new XMM-Newton data. Changes in the accretion geometry on longer time scales and in the mass accretion rate might cause the irregular light curves of October / November 1992 and on August 17, 2005 and the occurrence of a new sharp dip in the minima of the $V$-band light curves.

While the system geometry remains uncertain on the whole, the most likely scenario is a white dwarf with one main accreting pole undergoing a selfeclipse of the accretion region. This interpretation is consistent with the phasing of the UV and the optical light curves as well as with the results of our phase-resolved spectroscopic analysis. The permanent hard X-ray emission, nearly constant over the whole orbital cycle including the faint phase, might be a sign for the existence of a second independent accretion region in AI Tri, radiating in the hard X-ray band at a very low level. The unusual phasing of the stream dip when referring to the spectroscopic ephemeris of Schwarz et al. (1998) cannot be surely explained on the basis of the available data. Future optical spectroscopy is necessary to decide on the location of the accretion pole(s) and a potential slightly asynchronous rotation of the system. 


\section{CHAPTER 6}

\section{QS Tel - A polar changing state}

QS Tel is another source in the sample of polars that have been revealed in the ROSAT All-Sky Survey, independently in soft X-rays by Beuermann \& Thomas (1993) and in the EUV by Buckley et al. (1993), and shows a distinct soft X-ray flux component. With an orbital period of $2.33 \mathrm{hrs}$, it is the first one discovered among the few systems which settle the period gap of cataclysmic variables. From phase-resolved spectroscopy during intermediate and high accretion states, Schwope et al. (1995) derive the linear spectroscopic ephemeris

$$
\mathrm{HJD}=2448894.5568(15)+0.09718707(16) \cdot E
$$

with the blue-to-red zero crossing of the narrow emission line component at $\varphi=0.0$ (inferior conjunction of the secondary), which approximately corresponds to the maximum redshift of the high-velocity line component from the accretion stream. Undergoing frequent changes between high and low states and occasionally switching between one-pole accretion (e.g. Buckley et al. 1993) and two-pole accretion (e.g. Rosen et al. 1996), it exhibits a variety of accretion behavior and has been subject to several multi-wavelength studies. The epochs of one-pole and of two-pole accretion manifest themselves in the $\mathrm{X}$-ray and EUV light curves altering between one pronounced maximum per orbital cycle and a double-peaked shape. Schwope et al. (1995) suggest timedependent accretion onto one accretion pole close to the connecting line to the secondary, mainly emitting in the soft X-ray regime, and onto one optically bright accretion pole. This interpretation conforms to Rosen et al. (2001) who report on offsets in time between optically bright and EUV bright states. During modes of accretion mainly onto the primary, EUV bright pole, a recurrent sharp dip interrupts the soft X-ray and EUV light curves in the $\varphi_{\text {spec }}=0.0-0.1$ interval, described for instance by de Martino et al. (1998) and Rosen et al. 
(2001) and explained by the accretion stream crossing the line of sight in front of the heated surface area on the white dwarf.

The transitions from high to low accretion states are characterized by phaseshifts in the EUV light curves (Rosen et al. 2001) and by the optical flux maximum moving towards longer wavelengths (Gerke et al. 2006). The phase shifts may be triggered by changes in the accretion geometry, for instance by a migration of the accretion pole(s) to a different longitude (Rosen et al. 1996, 2001). Warren et al. (1993) find flare-like events during a low state of accretion, which they explain as the potential impact of accretion blobs at a low mass transfer rate or as magnetic flares. Short-time variability in the ultraviolet range (de Martino et al. 1998) provides further evidence of clumpy accretion events, as typical of soft X-ray dominated systems.

The magnetic field strength of the white dwarf in QS Tel ranges among the highest values measured in AM Her-type systems. Schwope et al. (1995) assign $B_{1} \sim 47 \mathrm{MG}$ to the EUV bright primary pole and $B_{2} \sim 70-80 \mathrm{MG}$ to the optically bright pole. The inclination of the system has not been clearly revealed yet. While Ferrario et al. (1994) and Schwope et al. (1995) derive values below $50^{\circ}$, Rosen et al. (1996) propose a high inclination of $i \sim 50-75^{\circ}$.

\subsection{XMM-Newton and optical observations}

During an intermediate high state of accretion, a $20 \mathrm{ksec}$ observation of QS Tel was carried out with XMM-Newton on September 30, 2006 (archived under observation ID 0404710401). The EPIC/MOS2 detector was operated in small window mode with the thin filter, EPIC/pn in large window mode with the medium filter. EPIC/MOS1 suffered from a full scientific buffer in timing mode and collected too little signal to perform an expedient analysis. The Optical Monitor was used simultaneously for ultraviolet fast mode photometry in the $2050-2450 \AA$ band with the UVM2 filter. We employed SAS v8.0 standard tasks for the data reduction and the effective area correction according to Section 4.2. A circular source region with a radius of 25 arcsec has been chosen. Due to gaps in the detector plane close to the source, the EPIC/MOS background was determined from a source-free region on an outer CCD, while the EPIC/pn background could be taken from the same chip as the source. The light curves have been binned to $30 \mathrm{~s}$ and $100 \mathrm{~s}$ intervals, the spectra to bins comprising a minimum of 20 counts. 
Table 6.1: Log of the XMM-Newton and optical observations of QS Tel on September 30 and October 1, 2006.

\begin{tabular}{lccrl}
\hline \hline Instrument & Filter & $\begin{array}{c}t_{\text {cycle }} \\
{[\mathrm{s}]}\end{array}$ & $\begin{array}{c}\text { Duration } \\
{[\mathrm{hrs}]}\end{array}$ & Observer \\
\hline XMM-Newton EPIC/pn & Thin & & 5.4 & PI Reinsch \\
XMM-Newton EPIC/MOS1 & Medium & & 1.8 & PI Reinsch \\
XMM-Newton EPIC/MOS2 & Medium & & 5.7 & PI Reinsch \\
XMM-Newton OM & UVM2 & & 5.0 & PI Reinsch \\
SMARTS CTIO 1.3m & $B$ & 100 & 2.0 & Walter \\
SMARTS 1.5m R-C & $3500-5300 \AA$ & 214 & 1.9 & Walter \\
\hline
\end{tabular}

Contemporary optical observations have been performed at the CTIO observatory of the SMARTS ${ }^{1}$ consortium on September 30 and October 1, 2006. The $B$-band photometry with the ANDICAM at the $1.3 \mathrm{~m}$ telescope covers almost one orbital period with a cycle time of $100 \mathrm{~s}$. Phase-resolved optical spectra in the $3500-5300 \AA$ range were obtained with the R-C spectrograph at the $1.5 \mathrm{~m}$ telescope during about $80 \%$ of an orbital cycle. They show a spectral resolution of $4.5 \AA$ in FWHM and a time resolution of $214 \mathrm{~s}$.

All times have been corrected to the barycenter. Table 6.1 summarizes the observational data of QS Tel.

\subsection{Radial velocity variations}

The optical spectra of QS Tel, obtained at the SMARTS $1.5 \mathrm{~m}$ telescope simultaneously to the XMM-Newton observations, show the typical Balmer and helium emission lines. Due to the system being in an intermediate high state of accretion and to the moderate spectral resolution, the lines are less prominent than in former high-state spectroscopy, presented for instance by Buckley et al. (1993) and Schwope et al. (1995).

From the optical phase-resolved spectroscopy, covering $80 \%$ of the orbital period, we estimate radial velocities in order to investigate the spectroscopic ephemeris. After identifying the emission line positions by eye, the spectra are shifted to the zero radial velocity and co-added. Gaussian fits to the cross correlation function between the individual spectra and the co-added template

\footnotetext{
${ }^{1}$ Small and Moderate Aperture Research Telescope System
} 


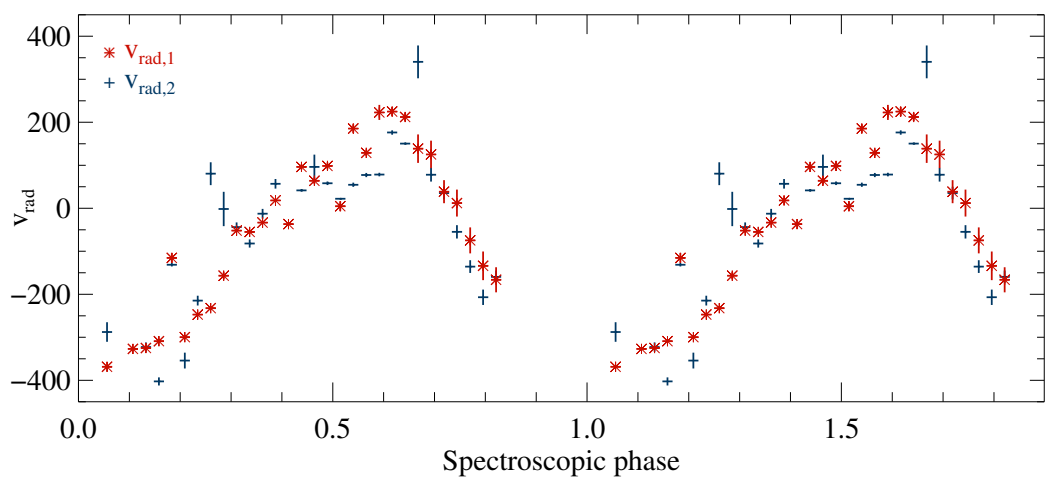

Figure 6.1: Radial velocity variations of the $\mathrm{H} \beta \lambda 4861 \AA$ line in the phase-resolved optical spectra of QS Tel, plotted twice for clarity. Two components of the emission lines that probably can be attributed to different areas in the accretion stream have been approximated with double-Gaussian fits.

give the radial velocities at a phase resolution of $\Delta \varphi=0.025$. The radial velocity curves show the large velocity amplitude $K>300 \mathrm{~km} \mathrm{~s}^{-1}$ with excesses to even higher values close to the maximum and the asymmetric, non-sinusoidal shape described by Buckley et al. (1993) and Schwope et al. (1995).

These authors distinguish two to three components in the emission lines, which they attribute to regions of different velocity in the accretion stream and to the secondary star. According to Schwope et al. (1995), the highvelocity component and the narrow emission line component cross each other at $\varphi \sim 0.3$ and $\varphi \sim 0.7$. In our low-resolution spectroscopy, different line components can be identified, but not clearly separated throughout all the exposures. The narrow emission line component, attributed to the irradiated secondary by Schwope et al. (1995), is barely discernible due to the lower line intensities and spectral resolution in our exposures. Figure 6.1 shows the result of cross correlation and double-Gaussian fit to two components of the $\mathrm{H} \beta \lambda 4861 \AA$ emission line that most probably originate in the accretion stream.

Since the radial velocity curves conform well with the results of Schwope et al. (1995) both in their shape and in the phasing, we consider their ephemeris (Equation 6.1) as accurate enough to be still valid for the 2006 September 30 observations, and refer to them throughout the analysis. In particular, we find no evidence of a quadratic term and thus an orbital period change that Schwope et al. (1995) discuss. 


\subsection{Multi-wave band photometry}

Figure 6.2 shows the EPIC/pn photometric data folded with the orbital period according to the spectroscopic ephemeris of the narrow emission line components derived by Schwope et al. (1995, Equation 6.1). While the soft X-ray emission is characterized by a distinct bright/faint behavior with one bright phase per orbital period, the flux at energies above $2 \mathrm{keV}$ is very low, but nonzero. At energies between 0.5 and $2.0 \mathrm{keV}$, the count rate is lower and the switch between bright and faint phases less pronounced than in the softest energy range. The main light curve shapes are similar during both the orbital periods covered, but deviate from each other in details. During the first cycle, the bright phase is more intense in the $0.5-2.0 \mathrm{keV}$ band than during the second one and less intense in the $0.1-0.5 \mathrm{keV}$ band. The light curves moreover differ in their flaring variability during the two cycles. In all the three energy ranges displayed in Figure 6.2, the bright phases cover about $40 \%$ of the orbital period, and they are most prominent in the soft X-ray regime below $0.5 \mathrm{keV}$. The maxima show a smooth rise and steep decline, and are two-fold disrupted by a descent in the flux: A broader, shallow dip lasting $\Delta \varphi \sim 0.08$ occurs around orbital phases $0.7-0.9$; a second, sharp dip at $\varphi=0.05$ appears to be restricted to the very soft X-ray component at energies between 0.1 and $0.5 \mathrm{keV}$. During the second dip with $\Delta \varphi<0.02$, the count rate nearly drops to zero, and the hardness ratio tends to one (Figure 6.3). The dips are seen during both the bright phases covered, but differ in depth.

The ultraviolet and the optical light curves show no clear modulation with the orbital phase and resemble the $V$-band light curves during low state obtained by Ferrario et al. (1994). This appearance is in contrast to the diverse highly variable shapes in former optical photometry during intermediate and high states of accretion, e. g. in the Buckley et al. (1993) and Rosen et al. (1996) SAAO and in the de Martino et al. (1998) HST data (for a more detailed description of light curve profile changes in QS Tel see also Rosen et al. 2001). Figure 6.4 displays the XMM-Newton Optical Monitor and the SMARTS $B$-band data in comparison to the simultaneously obtained mean EPIC/pn light curve. Slight modulations with the orbital period, in phase with the X-ray light curves, may be revealed after rebinning the optical data to intervals of $300 \mathrm{~s}$ (lowest panel in Figure 6.4). 


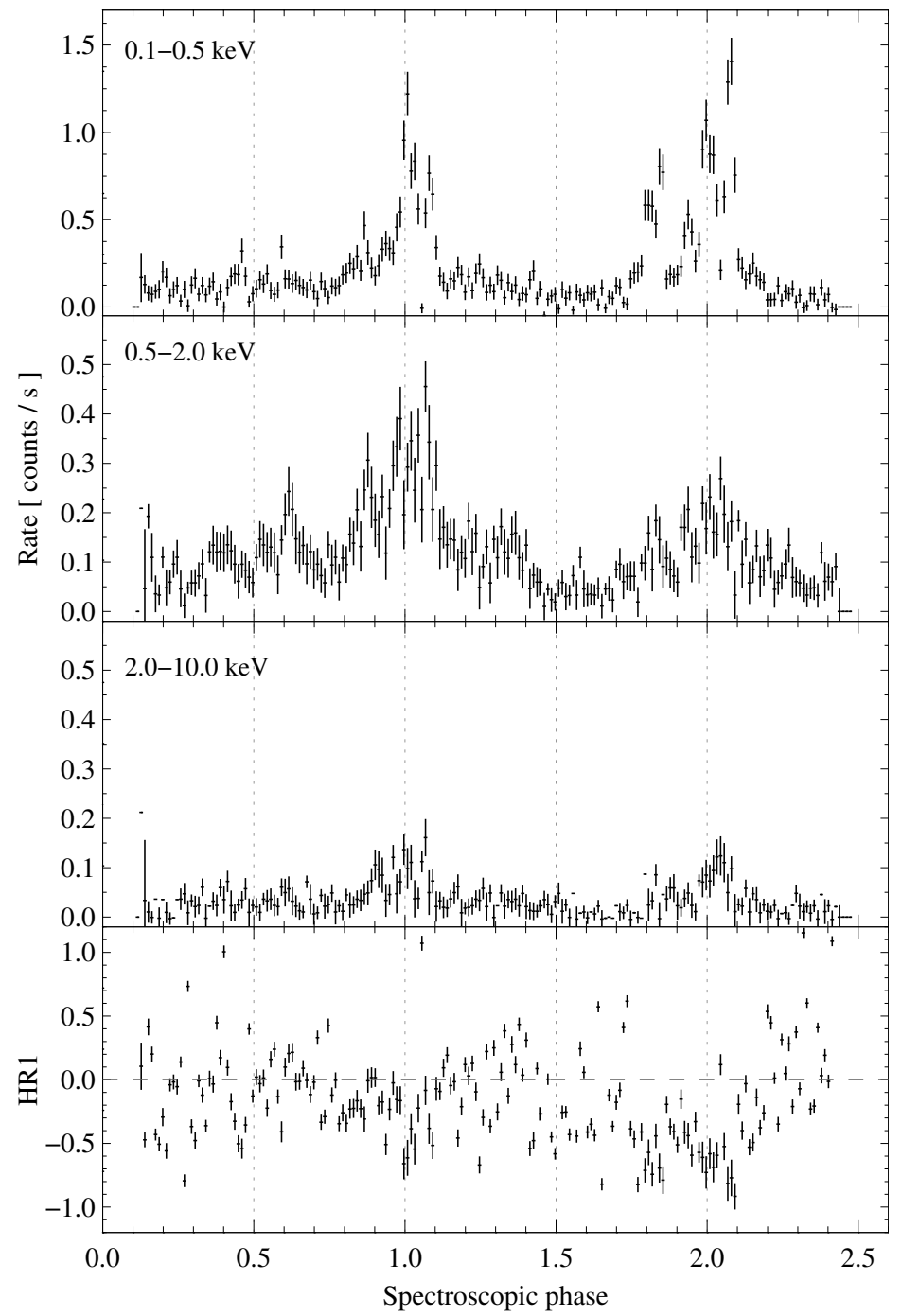

Figure 6.2: EPIC/pn light curves of QS Tel in different energy bands, folded with the orbital period according to the spectroscopic ephemeris from Schwope et al. (1995). The lowest panel shows the hardness ratio $\mathrm{HR} 1=(\mathrm{H}-\mathrm{S}) /(\mathrm{H}+\mathrm{S})$ between the $0.1-0.5 \mathrm{keV}$ and the $0.5-2.0 \mathrm{keV}$ band. Time bins are $100 \mathrm{~s}$. 


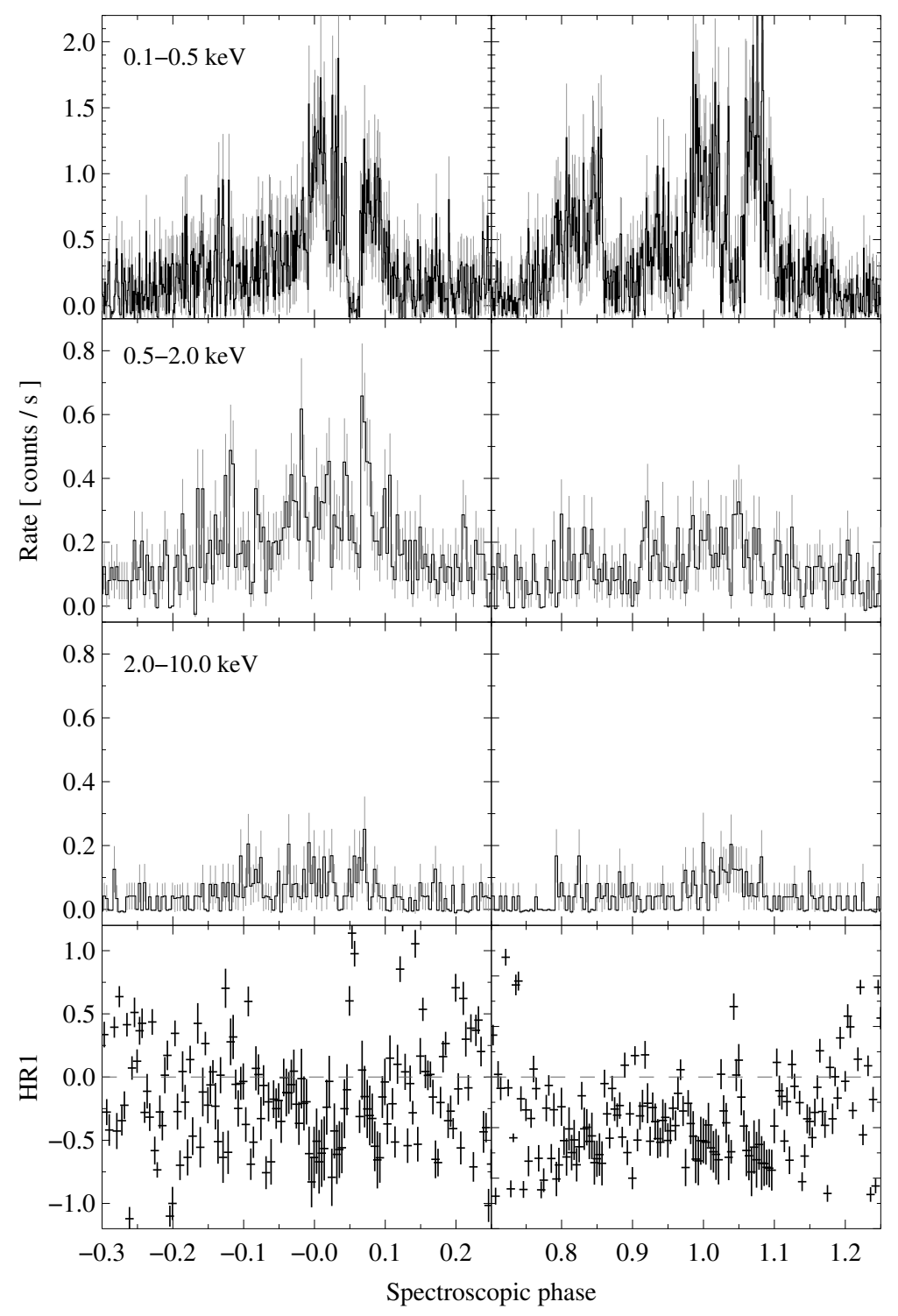

Figure 6.3: QS Tel EPIC/pn light curves during the bright phases in time bins of $30 \mathrm{~s}$, and of $10 \mathrm{~s}$ in the $0.1-0.5 \mathrm{keV}$ band. Wave bands and HR1 as in Figure 6.2. 


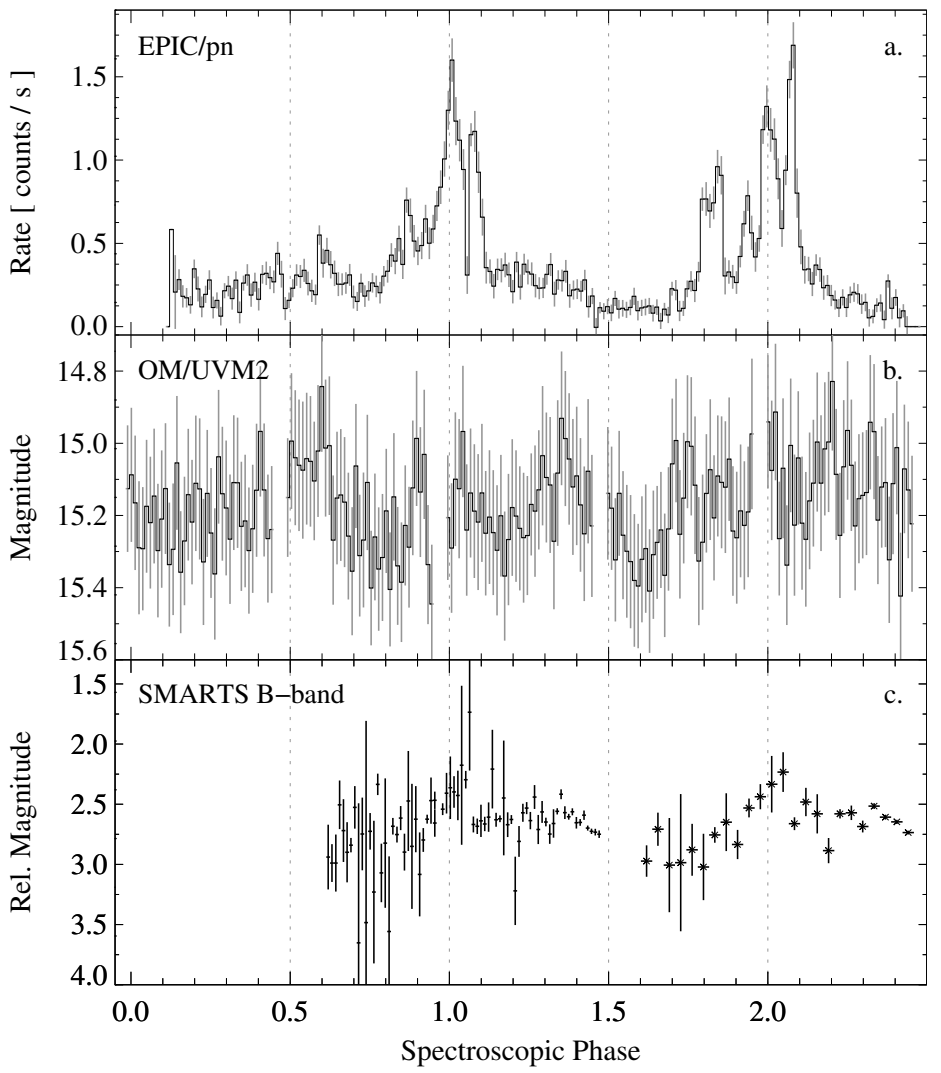

Figure 6.4: $a$. Mean EPIC/pn X-ray light curve, integrated over the complete energy range $0.1-10.0 \mathrm{keV}$. $b$. The ultraviolet XMM-Newton OM light curve in the $2050-2450 \AA$ band. Time bins are 100 s. c. Optical SMARTS light curve, obtained simultaneously to the XMM-Newton observation. The data have been plotted twice, to the left with the original cycle time of $100 \mathrm{~s}$, to the right rebinned to intervals of $300 \mathrm{~s}$. 


\subsection{The XMM-Newton/EPIC spectra}

Background subtracted X-ray spectra of QS Tel have been extracted from the $20 \mathrm{ksec}$ XMM-Newton exposure. We fit the EPIC/pn and EPIC/MOS2 spectra simultaneously in XSPEC v12.5 (Arnaud 1996; Dorman et al. 2003). Like in the spectral models to the XMM-Newton data of AI Tri (Section 5.3), black body and MEKAL components, for which we employ the abundances of Grevesse et al. (2007), have been combined with twofold absorption terms: TBNEW to account for the galactic absorption, using the cross-sections of Verner \& Ferland (1996) and Verner et al. (1996) and the abundances of Wilms et al. (2000); and PCFABS, partially covering the plasma emission, to represent the intrinsic absorption (for details on the XSPEC models see Section 3.1).

\subsubsection{The orbital mean spectrum}

QS Tel was in an intermediate high state of accretion during the XMM-Newton observation, and about a factor of 20 fainter than expected in high state from the ROSAT All-Sky Survey (Voges et al. 1999). The spectral fits thus result in relatively low black body and plasma temperatures, at which rich metal lines arise at energies above $0.3 \mathrm{keV}$. The soft $\mathrm{X}$-ray range $(0.1-0.4 \mathrm{keV})$ is well described by a single black body at $k T_{\mathrm{bb}}=21.2_{-3.1}^{+3.6} \mathrm{eV}$, which originates from the heated accretion region on the white-dwarf surface and is barely affected by interstellar absorption $\left(N_{\mathrm{H}, \mathrm{ISM}}=2.0_{-2.0}^{+4.1} \cdot 10^{19} \mathrm{~cm}^{-2}\right)$. Thermal plasma emission from the shocked accretion column shapes the harder X-ray spectrum above $0.4-0.5 \mathrm{keV}$. The approximation with a single MEKAL spectrum at a mean temperature of $k T_{\text {MEKAL }}=2.1_{-0.2}^{+0.4} \mathrm{keV}$ and an element abundance of $0.4_{-0.1}^{+0.2}$ times solar yields a modest $\chi_{\text {red }}^{2}=1.2$ at 181 degrees of freedom. Additional plasma components increase the fit accuracy (Table 6.2), indicating the need for multi-temperature models. We test different temperature structures using the XSPEC models CEMEKL and MKCFLOW (Section 3.1), and the multi-MEKAL models following Fischer \& Beuermann (2001, Section 3.3) and display the results in Figure 6.6. Neither the exponential temperature structure of the CEMEKL model nor the MKCFLOW cooling flow approach fit the hard component better than a single MEKAL. The multi-MEKAL model improves the fit quality marginally when using a low magnetic field strength of $B \simeq 10 \mathrm{MG}$ and a mean mass flow density of $\dot{m}=1 \mathrm{~g} \mathrm{~cm}^{-2} \mathrm{~s}^{-1}$. At these values, the temperature distribution over the height of the accretion column is rather flat (Figure 3.3). The slight amelioration mainly is visible in the ap- 


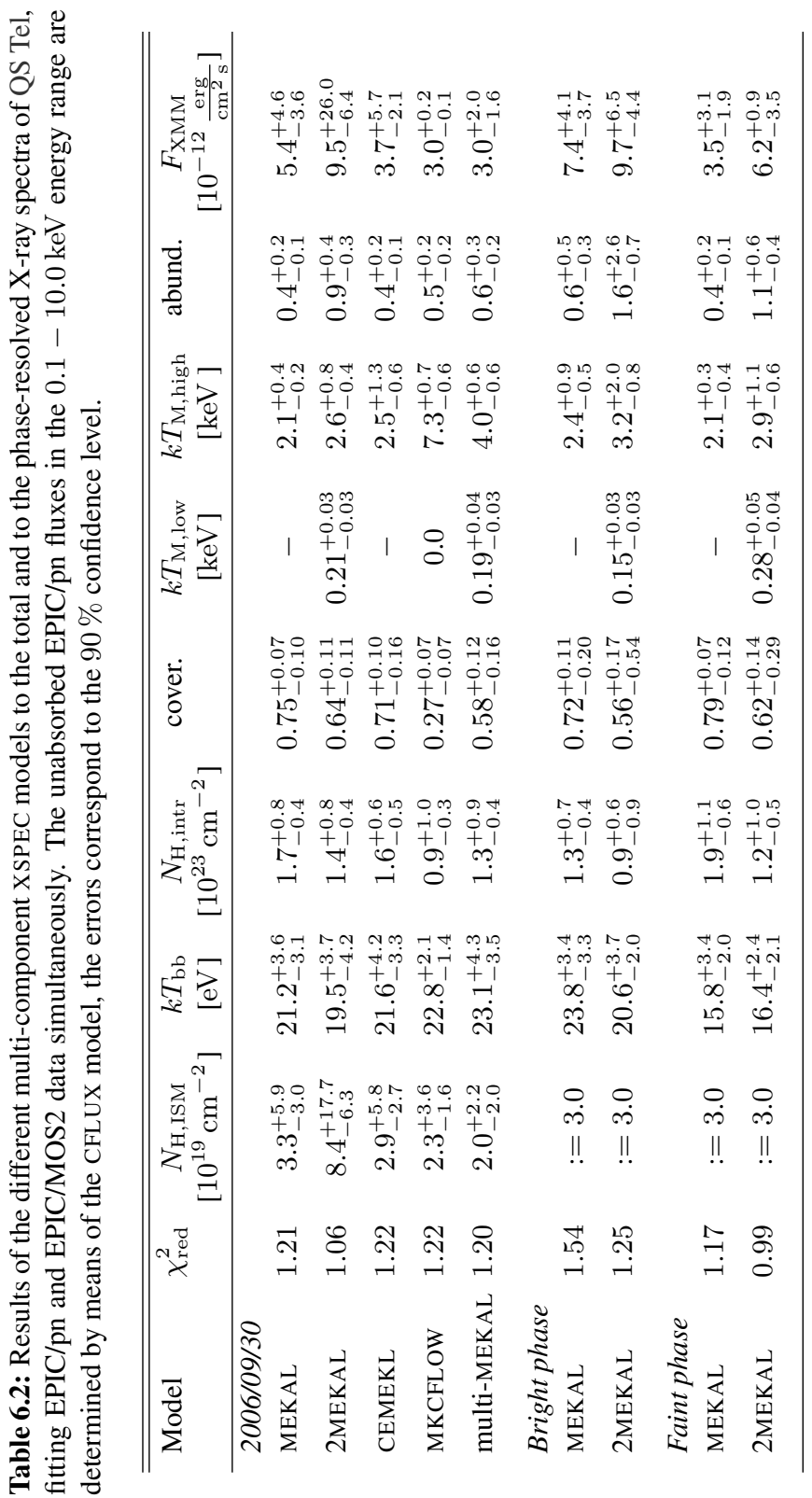




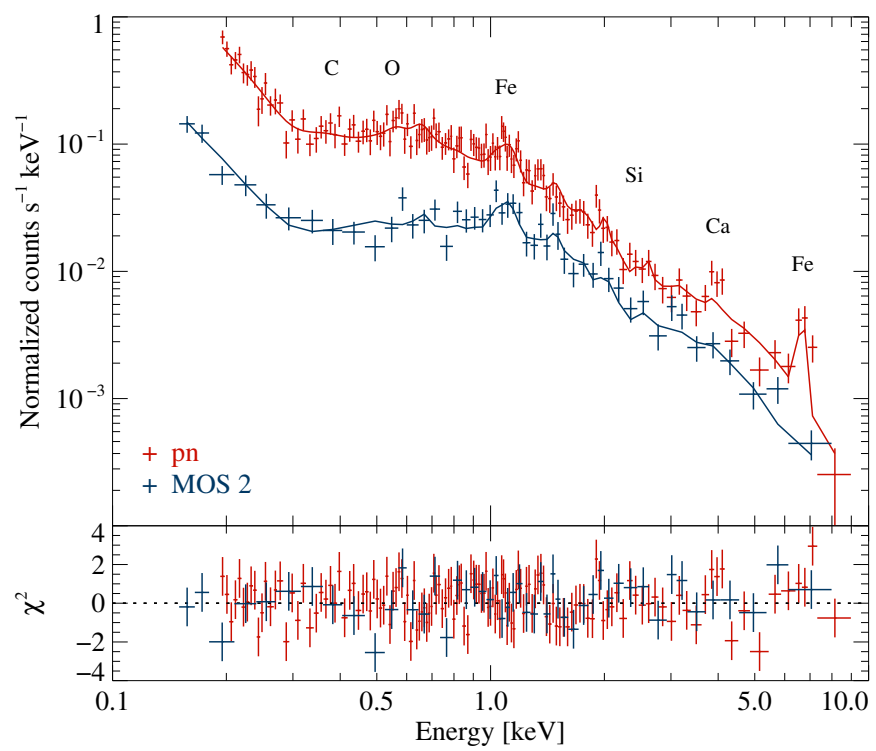

Figure 6.5: QS Tel EPIC spectra compared to the best-fit XSPEC model consisting of a single black body plus two partially absorbed MEKAL components.

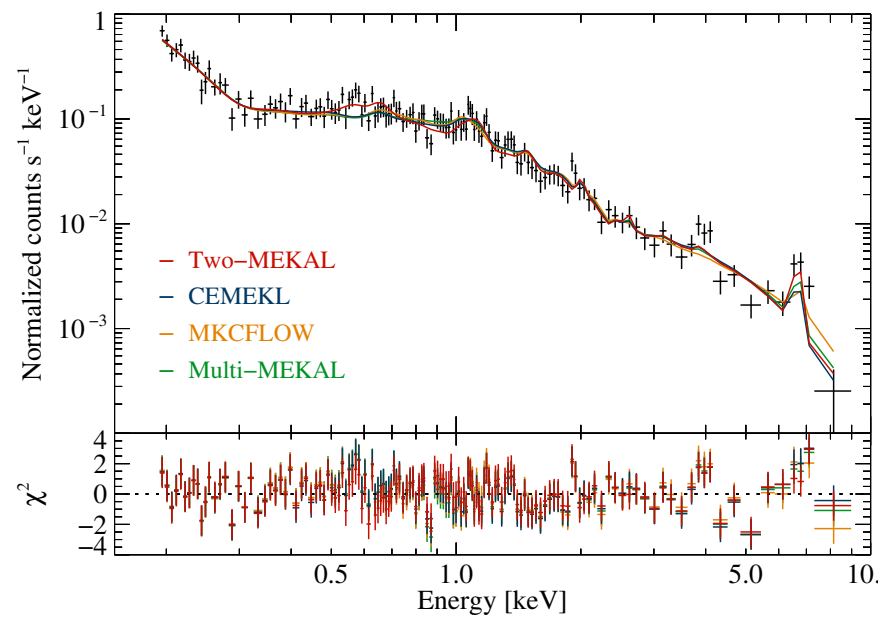

Figure 6.6: Different multi-temperature fits to the EPIC/pn spectrum of QS Tel. 
proximation of the $\mathrm{Ca} 4.6 \mathrm{keV}$ and the $\mathrm{Fe} 6.7 \mathrm{keV}$ lines. Hence, none of these approaches can satisfyingly reproduce the temperature structure in the plasma component.

The element abundances resulting from the different fits deviate from each other by a factor of up to two, with a clear trend to subsolar values. The strong correlation between plasma temperature, mass accretion rate, and element abundance in the models cause large systematic uncertainties when determining the chemical composition of the accretion stream.

Two MEKAL components with the same partial covering absorption term and identical element abundance give the best fit to the plasma component with a reduced $\chi_{\text {red }}^{2}=1.06$ at 180 degrees of freedom. It yields MEKAL temperatures of $k T_{\mathrm{MEKAL}, \text { low }}=0.21_{-0.03}^{+0.03} \mathrm{keV}$ and $k T_{\mathrm{MEKAL}, \mathrm{high}}=2.6_{-0.4}^{+0.8} \mathrm{keV}$ at a mean element abundance of $0.9_{-0.3}^{+0.4}$ times solar and an intrinsic absorption of $1.4_{-0.4}^{+0.8} \cdot 10^{23} \mathrm{~cm}^{-2}$ (Table 6.2). The almost solar metal abundance gives the best description of the oxygen and iron lines (Figure 6.6), but cannot reproduce the prominent calcium emission around $4.6 \mathrm{keV}$. The discrepancy may indicate a non-solar element distribution or different excitation mechanisms.

\subsubsection{X-ray bright phases}

The bright phases, defined by the on-off-behavior of the light curves in the softest energy range (Section 6.3), cover the phase interval $\varphi=0.7-1.1$. The absorbed black body plus MEKAL model with $N_{\mathrm{H} \text {,intr }}=1.3_{-0.4}^{+0.7} \cdot 10^{23} \mathrm{~cm}^{-2}$, $k T_{\text {MEKAL }}=2.4_{-0.5}^{+0.9} \mathrm{keV}$, and an $0.6_{-0.3}^{+0.5}$ times solar metal abundance resemble the fits to the orbital mean spectrum. With $F_{\mathrm{XMM}}=7.4_{-3.7}^{+4.1} \cdot 10^{-12}$ erg $\mathrm{cm}^{-2} \mathrm{~s}^{-1}$ in the XMM-Newton energy range, the fit yields a slightly enhanced model flux. We assume the TBNEW term to represent the interstellar absorption and hence expect it to stay constant around $N_{\mathrm{H}, \mathrm{ISM}}=3 \cdot 10^{19} \mathrm{~cm}^{-2}$ during all phase intervals, as derived from the orbital mean (Section 6.4.1). This approach results in $k T_{\mathrm{bb}}=23.8_{-3.3}^{+3.4} \mathrm{eV}$. The black body temperature increases only slightly, when the TBNEW component is allowed to vary anyhow $\left(N_{\mathrm{H}, \mathrm{ISM}}=1.2_{-1.2}^{+3.4} \cdot 10^{19} \mathrm{~cm}^{-2}\right)$, for example due to other effects contributing to the absorption of the soft spectral component. An additional MEKAL component for the hard energy band, as used in Section 6.4.1, reduces the $\chi_{\text {red }}^{2}$ of the fit from 1.54 to 1.25 , but is affected with large uncertainties in the higher MEKAL temperature and particularly in the element abundance.

The energy-resolved light curves in Section 6.3 reveal a broad dip around $\varphi=0.88-0.96$ and a sharp dip at $\varphi=0.05$. Fits to the spectra that have been 


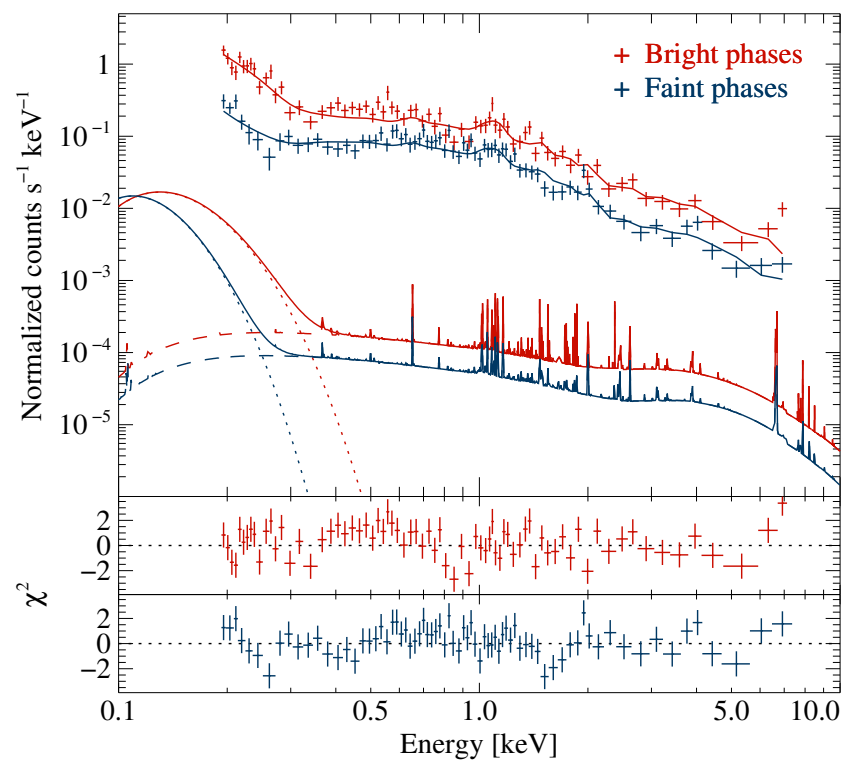

Figure 6.7: EPIC/pn spectra of QS Tel during the bright and the faint phases together with the black body (dotted) plus single MEKAL (dashed) models.

extracted from the different sections of the bright phases result in largely identical values for all parameters, including the absorption terms. The hardness ratio determined from the $\mathrm{X}$-ray photometry yet indicates a possible absorption effect: Additional intrinsic absorption on the order of $N_{\mathrm{H}, \text { intr }} \gtrsim 10^{24} \mathrm{~cm}^{-2}$ were necessary to reproduce the observed increase in the hardness ratio during the short dip, if the temperatures are assumed to be constant over the whole orbital cycle. In order to reveal the intrinsic $N_{\mathrm{H}}$ variations in the phase-resolved spectral fits, a significantly higher count rate were required.

\subsubsection{X-ray faint phases}

The X-ray spectra during the faint phases, covering the $\varphi=0.1-0.7$ interval, show at energies above $0.5 \mathrm{keV}$ a shape similar to the bright-phase spectra. MEKAL temperatures and abundances and the intrinsic absorption fairly agree within their error bars during all phase intervals, the faint-phase absorption tending towards higher and the MEKAL temperature towards lower values. The 
soft part of the spectrum $(0.1 \mathrm{keV} \leq \mathrm{E} \leq 0.5 \mathrm{keV})$ is slightly shifted towards lower fluxes, resulting in a cool black body temperature of $k T_{\mathrm{bb}}=15.8_{-2.0}^{+3.4} \mathrm{eV}$ which in particular conforms to the EPIC/MOS2 spectrum. In this model, the TBNEW absorption is again fixed to $N_{\mathrm{H}, \mathrm{ISM}}=3 \cdot 10^{19} \mathrm{~cm}^{-2}$. When treating the TBNEW absorption as a free parameter $\left(N_{\mathrm{H}, \mathrm{ISM}}=1.1_{-0.8}^{+1.6} \cdot 10^{20} \mathrm{~cm}^{-2}\right)$, the black body temperature reaches even lower values of $k T_{\mathrm{bb}}=12.6_{-2.9}^{+2.2} \mathrm{eV}$. The model flux of $F_{\mathrm{XMM}}=3.5_{-1.9}^{+3.1} \cdot 10^{-12} \mathrm{erg} \mathrm{cm}^{-2} \mathrm{~s}^{-1}$ ranges moderately below the mean values in the complete XMM-Newton energy range.

\subsection{Discussion and conclusions}

\subsubsection{The soft X-ray excess}

Both the X-ray light curves and the spectra evince the very soft X-ray flux of QS Tel. The count rate decreases rapidly towards higher energies with means of $\overline{\mathrm{CR}}_{1, \mathrm{br}}=0.45 \pm 0.07 \mathrm{cts} \mathrm{s}^{-1}$ in the $0.1-0.5 \mathrm{keV}$ band during the bright phases (orbital mean $\overline{\mathrm{CR}}_{1}=0.24 \pm 0.06 \mathrm{cts} \mathrm{s}^{-1}$ ), $\overline{\mathrm{CR}}_{2, \mathrm{br}}=0.21 \pm 0.04 \mathrm{cts} \mathrm{s}^{-1}$ in the $0.5-2.0 \mathrm{keV}$ and $\overline{\mathrm{CR}}_{3, \mathrm{br}}=0.14 \pm 0.02 \mathrm{cts} \mathrm{s}^{-1}$ in the $2.0-10.0 \mathrm{keV}$ energy bands. The hardness ratios, positive over large parts of the orbital cycle, and the soft-to-hard flux averages thus reflect the little spectral contribution of events at energies above $0.5 \mathrm{keV}$. The upper and lower flux limits of the integrated unabsorbed model components give a ratio of $F_{\mathrm{bb}} / F_{\mathrm{MEKAL}}=20-350$. The upper limit of the total integrated flux is $F_{\mathrm{bol}} \leq 6.3 \cdot 10^{-10} \mathrm{erg} \mathrm{cm}^{-2} \mathrm{~s}^{-1}$ and of the bolometric model luminosity $L_{\mathrm{bb}}=7 \cdot 10^{30}(d / 176 \mathrm{pc})^{2} \mathrm{erg} \mathrm{s}^{-1}$, using the distance determination $d=176_{-51}^{+39} \mathrm{pc}$ of Ak et al. (2007). These values are substantially lower than the results from the EUVE high state observations of Rosen et al. (1996), but on the same order as those from their ROSAT data, which they obtained during an intermediate high state of accretion. As a higher mass accretion rate is expected to affect the soft component mainly, the intermediate hardness and flux ratios can serve as lower limits for the high-state cases.

A distinct soft X-ray excess is most probably connected to the "blobby' accretion scenario (Section 2.5.3, Frank et al. 1988; Beuermann 2004), manifesting itself in the flaring structure of the X-ray light curves. Despite the moderate count rate during the XMM-Newton pointing, individual flare events with a typical duration of $10-15 \mathrm{~s}$ appear in the soft X-ray light curves, mostly during the bright phases (see Figure 6.8). These events can be assigned to separate 


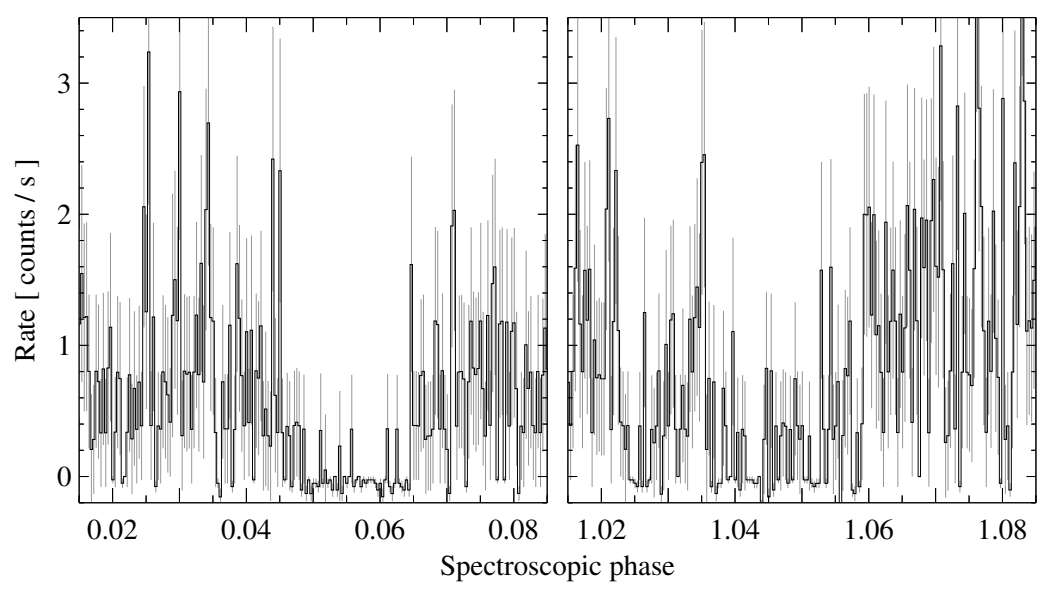

Figure 6.8: Flaring structure of the EPIC/pn light curve of QS Tel in the $0.1-0.5 \mathrm{keV}$ band around the sharp dips.

filaments in the accretion stream permeating the white-dwarf atmosphere at a low rate. The patterns are comparable to the flare light curves of V1309 Ori (Schwarz et al. 2005), which show - at a considerably higher count rate - clear evidence of single accretion blobs.

\subsubsection{The X-ray light curve dips}

The shape of the complex $20 \mathrm{ksec}$ X-ray light curves of QS Tel, shown in Figure 6.2, is similar to that of former ROSAT light curves presented by Schwope et al. (1995) and Rosen et al. (1996, 2001). The flux depression before and at spectroscopic phase 0.9 , well pronounced in the ROSAT PSPC light curve profile of Rosen et al. (1996), appears both in the $0.1-0.5 \mathrm{keV}$ and in the $0.5-2.0 \mathrm{keV}$ energy bands and varies in depth. Occasionally occurring, it might be an effect of random mass transfer variations as well as an absorption feature. A variable broader trough, preceding a short, deep light curve dip, is reported by Warren et al. (1995) for EUVE observations of UZ For. The authors consider absorption in an extended accretion column or structures in the accretion region as possible explanations for this broad dip.

Flare-like activity, previously stated by Rosen et al. (1996), characterizes the bright-phase light curves including the sharp dip. Figure 6.8 shows details of 
the EPIC/pn light curves in $3 \mathrm{~s}$ bins, centered around the two soft X-ray dips in the XMM-Newton observation. Possibly connected to the 'blobby' accretion as described in Section 6.5.1, flaring events may also account for the depth variations of the shallow and the sharp dips from cycle to cycle.

The recurring, narrow dip in the softest energy band at $\varphi=0.05$ was also found in former EUVE (Rosen et al. 1996, 2001) and UV observations (de Martino et al. 1998) and generally is explained by the accretion stream eclipsing the main pole. The short ingress and egress times and the hardness-ratio peak fit in this picture. Warren et al. (1995) discuss the possibility that the sharp dip in the EUVE light curves of UZ For originates from accretion stream absorption. They point out that the influence of mass accretion rate variations on the location of the threading region can explain why the depth of the dips changes between different orbital cycles. On the other hand, the interpretation as stream dip contrasts the conclusions which Schwope et al. (1995) draw from their optical spectroscopy. They assign the narrow emission line component to the irradiated secondary star and thus identify the blue-to-red zero crossing of the corresponding radial velocity curve at $\varphi=0.0$ with the inferior conjunction of the secondary. Typically, the main accretion regions in polars are located at longitudes below $90^{\circ}$ (Cropper 1988), which places a potential stream dip in the $\varphi=0.75-1.0$ interval in this phase convention, unlikely between $\varphi=0.05$ and $\varphi=0.10$. Romero-Colmenero et al. (2005) propose that eclipses of the X-ray bright accretion region by the secondary cause the light curve dips in the soft $\mathrm{X}$-ray and EUVE, while the optically bright accretion region is visible during the whole orbital cycle. In this case, the inferior conjunction is to be re-phased by $\Delta \varphi=-0.08$, and the lack of eclipse features in the optical data is plausible. De Martino et al. (1998), however, report on flux dips in their ultraviolet HST observations (1250-2500 $\AA$ ) that most probably reflect the optically (and UV) bright accretion pole. The system geometry should, hence, still be considered as preliminary.

\subsubsection{Accretion state}

Our multi-wavelength observations of QS Tel give another example for the versatile appearance of the system. The distinct bright phases of the X-ray light curves can be assigned to a main accretion pole, while the non-zero faint phase emission points to the existence of a second accretion region which is fed at a lower level. Schwope et al. (1995) establish the two-pole accretion geometry by identifying two sets of cyclotron lines in the optical spectra. The bright phases 
then can be ascribed to accretion activity onto the X-ray dominating pole. From the bolometric black body luminosity $L_{\mathrm{bb}}=5.6 \cdot 10^{31} \mathrm{erg} \mathrm{s}^{-1}(d=176 \mathrm{pc}$, Ak et al. 2007) during the bright phases, we estimate an intermediate mass accretion rate on the order of $\dot{M}=L_{\mathrm{bb}} \mathrm{R}_{\mathrm{WD}} / \mathrm{GM}_{\mathrm{WD}}=10^{-11} \mathrm{M}_{\odot} \mathrm{yr}^{-1}$ (on the equation, see e.g. Schwarz et al. 2005), consistent with the normalization of the MKCFLOW model $\dot{M}_{\text {MKCFLOw }}=1.8_{-0.8}^{+0.1} \cdot 10^{-11} \mathrm{M}_{\odot} \mathrm{yr}^{-1}$ (Section 6.4).

The little variability in the optical and ultraviolet wave bands can be attributed to the second, highly magnetic pole at a lower accretion rate. If this accretion pole shapes the faint-phase light curves and spectra, the black body component with a temperature of about $16 \mathrm{eV}$, lower than during the bright phases, arises from the less heated white-dwarf surface around the second accretion column. The spectral parameters indicate a mass accretion rate of approximately $\dot{M}_{\text {faint }} \sim 10^{-12} \mathrm{M}_{\odot \mathrm{yr}^{-1}}$ for the faint phases. Another twopole accreting polar with the second accretion region possibly seen during the $\mathrm{X}$-ray faint phase is BY Cam, one of the few asynchronous systems. Ramsay \& Cropper (2002) assign the flaring bright light curve intervals of BY Cam to a soft pole with 'blobby' accretion, and the low, hard faint phase flux to the second pole, the soft emission of which is shifted into the ultraviolet range. The authors state two alternative reasons for the lower temperatures in the accretion region, which would cause the wavelength shift: the region is less effectively heated due to a low accretion rate, or the accreted material is spread over a rather extended accretion area.

Alternatively, the faint-phase emission may stem from the main, X-ray bright accretion area in case it is not completely hidden behind the white dwarf during its orbital motion. The similar MEKAL temperatures and partial covering absorption terms in the phase-resolved spectral models (Section 6.4) then continuously reflected the main accretion column. The weaker soft X-ray emission in the faint-phase spectra could be explained by additional absorption of black body flux on the line of sight during the partial occultation of the accretion region, subsumed in $N_{\mathrm{H}, \mathrm{TBNEW}}=1.1_{-0.8}^{+1.6} \cdot 10^{20} \mathrm{~cm}^{-2}$. A signature of the second accretion pole in the XMM-Newton data hence is probable, but not definite. 


\section{CHAPTER 7}

\section{Summary and perspectives}

Since Rothschild et al. (1981) studied the X-ray spectral properties of AM Her, it is known that some AM Her-type cataclysmic variables show an enhanced soft X-ray flux. While the explanations with the 'blobby' accretion scenario (Kuijpers \& Pringle 1982), the concept of buried shocks, and the dominance of cyclotron cooling at high magnetic field strengths and low mass flow densities (see Beuermann \& Burwitz 1995) are widely accepted, the actual number of soft X-ray dominated systems is still under discussion. With the polars QS Tel and AI Tri, two examples which show a distinct soft-to-hard X-ray excess in the ROSAT All-Sky Survey have been studied in this work. The measured flux ratio depends on the underlying spectral model, which is determinant on which part of the spectrum is ascribed to which system component. Bremsstrahlung models for the hard part of the spectrum $(E \gtrsim 0.5 \mathrm{keV})$ for example, as typically used for ROSAT data, rapidly drop towards lower energies, while the plasma model of Cropper et al. (1999) includes low-temperature components and thus has a considerable part in the flux below $E \lesssim 0.5 \mathrm{keV}$.

\section{Preparatory work}

Accretion column and accretion region feature a complicated density and temperature structure. Though model approaches to different aspects exist, no consistent description has been achieved up to now due to the complexity of the problem. In order to approximate the wide parameter range, simplified semiempirical multi-temperature models for the soft and for the hard energy range have been employed in this work. The XSPEC model MKCFLOW, originally intended for cooling flows in galaxies, can reflect the thermal emission and the decreasing temperatures in the accretion column. Fischer \& Beuermann 
(2001) calculate the post-shock structure by solving stationary two-fluid hydrodynamic and radiative transfer equations. Their results have been adopted to supply an alternative multi-MEKAL model for the hard spectral component, which specifically considers the processes in a magnetically controlled accretion column. As an example for a multi-temperature description of the soft $\mathrm{X}$-ray emission from the accretion region, a Gaussian distribution of temperatures over the radius (giving the emitting surface areas) has been tested in the spectral analyses.

In preparation for a more accurate spectral fitting, the calibration of the EPIC instruments on-board XMM-Newton was examined in the soft energy range, where it is known to be least precise. A dedicated calibration object is the isolated neutron star RX J1856.4-3754, being established for the soft $\mathrm{X}$-ray regime in divers missions. It features a high soft $\mathrm{X}$-ray and a low optical flux with a plain spectrum that is well described by two black bodies (Beuermann et al. 2006). The EPIC/pn data of RX J1856.4-3754, which span more than five years, reveal a high calibration precision and stability over time. The deviations of the data-to-model ratios stay below an amplitude of $5 \%$. In the case of the EPIC/MOS detectors, the calibration stronger depends on the observation epoch, and larger discrepancies between observations and model occur with excesses of up to $20 \%$ for MOS2. As we restrict the application of these results to objects with very soft X-ray spectra, it is possible to neglect the dependence of the energy redistribution on the incident energy, and the deviations are corrected by adjusting the instrumental effective area.

Another factor that may detract from the quality of the analysis is the occurrence of soft proton flares as during one XMM-Newton observation of AI Tri. The investigation of the enhanced flaring background and its impact on exposures of bright objects amend former studies that concentrated more on extended and faint sources. In addition to the expected spectral softening, the signature of the flaring events can be identified in the spectra by an increased number of counts above $3-4 \mathrm{keV}$. The effects on the photometry and spectroscopy of bright sources can be neglected during phases of moderate background activity. Intervals of considerably high background - in the case of the AI Tri observation approximately 100 times as intense as the quiescent background - need to be excised from the analysis even for bright sources. 


\section{Observational results}

The main part of the work focused on the XMM-Newton observations of the soft polars AI Tri and QS Tel. Two pointings with exposure times of $20 \mathrm{ksec}$ and $1-5 \mathrm{ksec}$ respectively plus optical monitoring that spans ten nights within nineteen months provide the observational basis of the analysis of AI Tri (RX J0203.8+2959). From the $V$-band minima combined with former photometry of Schwarz et al. (1998), refined ephemeris of the system that apply to the complete observation epoch have been derived (Equation 5.1). A new feature in the $V$-band light curves is a dip at photometric phase $\varphi_{\text {phot }}=0.0$. The pronounced flickering in the optical light curves shows a systematic pattern which indicates quasi-periodic oscillations at periods between $5 \mathrm{~min}$ and $10 \mathrm{~min}$, slightly shorter than the $6.5 \mathrm{~min}$ and $14 \mathrm{~min}$ QPOs described by Schwarz et al. (1998). The X-ray light curves are governed by two bright phases, which we attribute to a single accretion region, undergoing eclipses by the accretion stream when it crosses the line of sight. With multi-temperature approaches both to the soft and to the hard part of the spectrum, the best spectral fit yields black body temperatures up to $44.0_{-3.2}^{+3.3} \mathrm{eV}$ and plasma temperatures between $0.8_{-0.2}^{+0.4} \mathrm{keV}$ and $20.3_{-5.1}^{+8.7} \mathrm{keV}$. The element abundance lies in the range of a solar distribution, when referring to the solar abundance ratio of Grevesse et al. (2007). This result exceeds the values that we derive when using the Anders \& Grevesse (1989) element distribution by about $20 \%$. The phase-resolved spectra mainly differ in the normalizations of the individual components and in the partial covering absorption terms, supporting the geometrical interpretation of the interrupted maximum as an eclipse of the accretion region by the stream. The spectroscopic phasing of the stream dip with respect to the shift between photometric and spectroscopic phase that Schwarz et al. (1998) derive either implies an unusual location of the main accretion pole on the white-dwarf surface or a slightly asynchronous rotation of the system. The lowered flux during the second part of the bright phase and a decrease in the optical line emission stated by Schwarz et al. (1998) could be effects of an extended accretion curtain. One $V$-band light curve and the first, short XMM-Newton observation were obtained during an 'irregular' accretion mode of AI Tri and are characterized by a phase shift of the optical minimum by $\Delta \varphi=0.2$, high variability, and a considerable spectral softening.

During an intermediate high state of QS Tel (RE J1938-462), a $20 \mathrm{ksec}$ XMM-Newton observation and optical photometry and spectroscopy were obtained simultaneously. The X-ray spectra have been modeled with a single 
black body - multi-temperature models are not applicable to the soft part due to the small number of counts - and different plasma components. The best spectral fit is achieved by two independent MEKAL models with a common absorption term at $N_{\mathrm{H} \text {,intr }}=1.4_{-0.4}^{+0.8} \cdot 10^{23} \mathrm{~cm}^{-2}, k T_{1}=0.21_{-0.03}^{+0.03} \mathrm{keV}$, and $k T_{2}=$ $2.6_{-0.4}^{+0.8} \mathrm{keV}$. The soft component, well described by a single black body at $k T_{\mathrm{bb}}=19.5_{-4.2}^{+3.7} \mathrm{eV}$, shows little absorption of $N_{\mathrm{H}, \mathrm{ISM}}=8.4_{-6.3}^{+17.7} \cdot 10^{19} \mathrm{~cm}^{-2}$. Relatively low column densities of the soft component and low temperatures were also reported by Rosen et al. (1996) from ROSAT and EUVE spectra. Both in the spectral and in the photometric data, the soft X-ray excess, although less pronounced than it would be expected for a high state of accretion, is obvious. Our data indicate a high accuracy of the Schwope et al. (1995) ephemeris and thus a high degree of synchronism, while the geometrical interpretation of $\varphi_{\text {orb }}=0$ (the blue-to-red zero-crossing of the radial velocities derived from the narrow emission line component) as inferior conjunction of the secondary remains ambiguous. The emission from one main accretion pole dominates the bright-phase X-ray data, while a second one with a low mass accretion rate may become visible during the faint phases. The little variability of the optical and ultraviolet light curves then is connected to the low activity of this second pole.

\section{Discussion}

The observed systems AI Tri and QS Tel, selected because of their low hardness ratios in the ROSAT All-Sky Survey, show a distinct dominance of soft over hard X-ray emission. The Gaussian temperature distribution gives an example for a multi black body model that accounts for an extended range of temperatures in the accretion region. In the spectra of the observed objects, the soft flux maximum lies outside the XMM-Newton energy range that commences at $0.19 \mathrm{keV}$ (EPIC/pn) and $0.15 \mathrm{keV}$ (EPIC/MOS) respectively. A decision on the best-fit model to the EPIC spectra is demanding, as the absorption term mostly compensates for the differences between single and multi black body models. The use of the multi-component black body yet improves the fit accuracy and better approximates the UV flux measured with the Optical Monitor. A good possibility to evaluate the models provide for example Chandra LETGS spectra, covering energies down to $70 \mathrm{eV}$.

With high magnetic field strengths of $B \sim 38 \mathrm{MG}$ (Schwarz et al. 1998) and $B_{1} \sim 47 \mathrm{MG}, B_{2} \sim 70-80 \mathrm{MG}$ (Schwope et al. 1995) respectively, AI Tri and QS Tel relate to the trend towards enhanced soft-to-hard flux ratios for 
increasing field strengths found by Beuermann \& Burwitz (1995). High variability characterizes their soft X-ray light curves as expected for systems the accretion flow in which has an inhomogeneous structure ('blobby' accretion scenario). Single flaring events in the light curves of QS Tel probably reflect the impact of individual dense filaments onto the white dwarf primary. Bolometric flux ratios during the bright phases of $F_{\mathrm{bb}} / F_{\mathrm{MEKAL}}=5.7_{-3.0}^{+6.1}$ and $F_{\mathrm{bb}} / F_{\text {MEKAL }}=20-350$ respectively place AI Tri and QS Tel amongst the objects with a strong soft X-ray excess. The highest values in XMM-Newton spectra, for comparison, are estimated for V1309 Ori with a bolometric soft-tohard flux ratio of several thousand (Schwarz et al. 2005) and for EU UMa with a bolometric luminosity ratio of $14-2800$ (Ramsay et al. 2004). In the few systems lacking distinct soft X-ray emission, as 2XMMp J131223.4+173659 (Vogel et al. 2008) and V2301 Oph (Ramsay \& Cropper 2007), the flux maximum of the reprocessed component appears to be shifted towards the EUV and UV regime (cf. Gänsicke 1998). The same could apply to objects like EV UMa (Ramsay \& Cropper 2003) which show a detectable, but very weak soft component. This interpretation implies that single black body models generally give only a lower limit for the reprocessed emission from the accretion region and thus for the flux ratio. The effect is demonstrated with the multi-temperature black body, which enhances the soft bolometric model flux during the bright phase of AI Tri by about $50 \%$, while the ultraviolet OM flux serves as an upper limit for the low-temperature components in the model. In order to quantify the soft X-ray excess, the multi-component models to the accretion column, hence, should be contrasted with adequate multi-temperature models to the soft X-ray emission region.

The multi-temperature fits to the plasma emission surpass the single MEKAL or bremsstrahlung approximation. MKCFLOW and multi-MEKAL models acceptably reflect the spectrum at energies above $0.5 \mathrm{keV}$. When interpreting parameters like the element abundance, the model dependence and strong correlation for example between abundance, mass, temperature, and particle density in the plasma models should be kept in mind. The temperature and density structures used in this work apply to sub-columns of identical mass flow densities and serve as good approximations to the complex real situation. A comprehensive description had to consider hydrodynamics, radiative transfer, and geometrical properties to subsume a wide range of local mass flow densities, temperatures, and particle densities. The spectral fits on the basis of the Fischer \& Beuermann (2001) approach, comprising hydrodynamic and radiative transfer equations, help to distinguish between possible temperature 
structures and parameter sets in order to confine the physical conditions in the emission regions. Observations over a broad range of energies allow for an additional determination of system properties and thus for a further restriction of the number of free parameters in the spectral models. Cyclotron lines in the optical spectra or optical polarimetry, for example, give the magnetic field strength of the white dwarf; photometry and spectroscopy from the ultraviolet to the infrared regime constrain the inclination. On the whole, the different multi-temperature approaches form first steps towards a more consistent modeling of the emission from the accretion column and from the accretion region in AM Her-type cataclysmic variables. 


\section{APPENDIX A}

\section{The multi-temperature black body model in XSPEC}

The multi-temperature black body model, described in Section 3.2 and implemented in XSPEC as an additional model, bases on a Gaussian distribution of temperatures over the radius. The Gaussian distribution is given by

$$
k T(r)=k T_{*}+\text { const. } \cdot e^{-\frac{\left(r-r_{1}\right)^{2}}{2 \sigma^{2}}}
$$

with the boundary conditions

$$
\begin{array}{lll}
k T \rightarrow k T_{\max } & \text { for } & r \rightarrow 0 \\
k T \rightarrow k T_{*} & \text { for } & r \rightarrow \infty
\end{array}
$$

In order to discretize Equation A.1, the accretion region is approximated by concentric rings of constant width $\delta$ (Figure A.1). Each ring, numbered $n$, has the temperature at its mean distance $r_{\mathrm{n}}$ from the region center. The maximum temperature is assigned to the innermost region at $n=1$. With

$$
r_{\mathrm{n}}=\frac{1}{2}(2 n-1) \cdot \delta
$$

and Equation A.2 follows for the inner circle at maximum temperature $k T_{1}$

$$
k T\left(r_{1}=\frac{1}{2} \delta\right)=k T_{*}+\text { const. } \cdot e^{-\frac{\delta^{2}}{8 \sigma^{2}} \stackrel{!}{=}} k T_{1}
$$

or

$$
\text { const. }=\left(k T_{1}-k T_{*}\right) \cdot e^{\frac{\delta^{2}}{8 \sigma^{2}}} .
$$

Thus, Equation A.1 becomes

$$
k T(r)=k T_{*}+\left(k T_{1}-k T_{*}\right) \cdot e^{-\frac{1}{2 \sigma^{2}}\left(r^{2}-\frac{1}{4} \delta^{2}\right)} .
$$



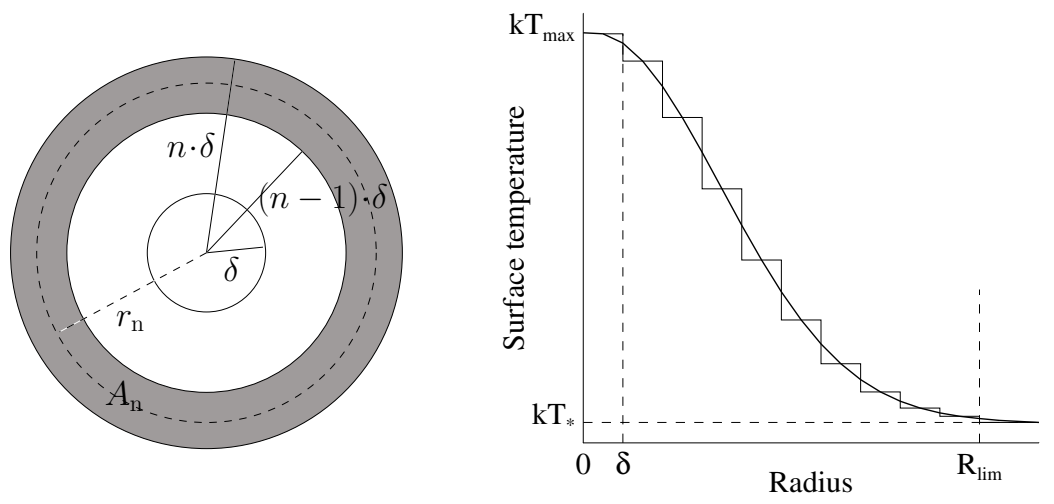

Figure A.1: Parametrization of the Gaussian temperature distribution. Left: Nomenclature of the concentric rings, used to derive the model parameters. Right: Temperature structure as a discretized Gaussian function of the radius.

In dependence on the ring number $n$, the mean temperature can be written as

$$
\begin{aligned}
k T_{\mathrm{n}} & =k T\left(r=\frac{1}{2}(2 n-1) \delta\right) \\
& =k T_{*}+\left(k T_{1}-k T_{*}\right) \cdot e^{-\frac{\delta^{2}}{2 \sigma^{2}} \cdot n(n-1)} .
\end{aligned}
$$

The outer radius $R_{\lim }=N_{\lim } \cdot \delta$ is defined by the decline of the temperature: $R_{\lim }$ is the radius, where the difference between the maximum temperature $k T_{1}$ at the center and the minimum temperature $k T_{*}$ at the white-dwarf surface reaches the fraction

$$
k T_{\lim }=x_{\lim } \cdot\left(k T_{1}-k T_{*}\right) .
$$

Equation A.8 allows the width $\sigma$ of the Gaussian to be written as a function of $x_{\lim }$ :

$$
x_{\lim }=e^{-\frac{\delta^{2}}{2 \sigma^{2}} \cdot n(n-1)}
$$

and

$$
-2 \sigma^{2}=\frac{\delta^{2}}{\ln x_{\lim }} \cdot N_{\lim }\left(N_{\lim }-1\right)
$$

Thus, using Equation A.7,

$$
\begin{aligned}
k T_{\mathrm{n}} & =k T_{*}+\left(k T_{1}-k T_{*}\right) \cdot e^{\frac{n(n-1)}{N_{\lim }\left(N_{\lim }-1\right)} \cdot \ln x_{\lim }} \\
& =: k T_{*}+\left(k T_{1}-k T_{*}\right) \cdot f(n)
\end{aligned}
$$


defines the temperature of the $n$th black body component in the additive model. The emitting surface area $A_{\mathrm{n}}$ of the ring numbered $n$, equivalent to the normalization of the BBODYRAD model, is

$$
A_{\mathrm{n}}=\pi\left(n^{2} \delta^{2}-(n-1)^{2} \delta^{2}\right) .
$$

Using the area $A_{1}=\pi \delta^{2}$ of the innermost circle, we obtain the dependence of the $n$th surface area on $A_{1}$ :

$$
\begin{aligned}
A_{\mathrm{n}} & =\pi \delta(n \delta+(n-1) \delta) \\
& =(2 n-1) A_{1}
\end{aligned}
$$

By means of the relations provided in Equations A.11 and A.12, the individual model components are coupled to the black body with the highest temperature. Equations 3.11 and 3.12 in Section 3.2 follow with a maximum number of fifteen rings and $x_{\lim }=1 \%$ as criterion for the outer boundary of the emission region. 
112

The multi-temperature black body model in XSPEC 


\section{APPENDIX B}

\section{Soft X-ray standard stars}

The following pages, supplementing Section 4.2, display the XMM-Newton observations of soft X-ray standard stars and the boresight effective area corrections applied to the EPIC instruments. Tables B.1 to B.3 summarize the available data sets. A cross marks the exposures which directly contribute to the correction functions for the soft X-ray sensitivity. Example spectra of RXJ1856.4-3754 before (blue) and after (red) applying the instrumental effective area correction are shown in Figures B.1 to B.3. The smaller panels comprise the corresponding data-to-model ratios and the effective area correction function. Uncertainties in the energy redistribution and a rapidly increasing instrumental background distort the spectra below $E \lesssim 0.18 \mathrm{keV}$ in a rather unpredictable way. 
Table B.1: Archival XMM-Newton EPIC/pn observations of the white dwarf HZ 43 A and the isolated neutron star RX J1856.4-3754.

\begin{tabular}{|c|c|c|c|c|c|c|}
\hline Cycle & Obs. ID & Date & Filter & Mode & \multicolumn{2}{|c|}{$t_{\text {exp }}[\mathrm{ksec}]$} \\
\hline \multicolumn{7}{|c|}{$H Z 43 A$} \\
\hline \multirow[t]{4}{*}{0089} & 0125970101 & $2000 / 06 / 04$ & Thin & Small window & 8.3 & \\
\hline & & & Medium & Small window & 8.3 & \\
\hline & & & Open & Small window & 8.3 & \\
\hline & & & Thick & Small window & 3.6 & \\
\hline 0928 & 0212090101 & $2005 / 01 / 03$ & Thin & Small window & 2.5 & \\
\hline \multicolumn{7}{|c|}{ RXJ1856 } \\
\hline 0427 & 0106260101 & $2002 / 04 / 08$ & Thin & Small window & 57.2 & $x$ \\
\hline 0798 & 0201590101 & $2004 / 04 / 17$ & Thin & Timing & 65.2 & \\
\hline 0878 & 0165971601 & $2004 / 09 / 24$ & Thin & Small window & 33.0 & $\times$ \\
\hline 0968 & 0165971901 & $2005 / 03 / 23$ & Thin & Small window & 35.0 & $x$ \\
\hline 0980 & 0213080101 & $2005 / 04 / 15$ & Closed & Extended ff & 7.7 & \\
\hline 1061 & 0165972001 & $2005 / 09 / 24$ & Thin & Small window & 33.1 & $x$ \\
\hline 1153 & 0165972101 & $2006 / 03 / 26$ & Thin & Small window & 69.6 & $\times$ \\
\hline 1259 & 0412600101 & $2006 / 10 / 24$ & Thin & Small window & 72.6 & $\times$ \\
\hline 1330 & 0412600201 & $2007 / 03 / 14$ & Thin & Small window & 66.3 & $\times$ \\
\hline 1335 & 0415180101 & $2007 / 03 / 25$ & Thin & Small window & 40.5 & \\
\hline 1432 & 0412600301 & $2007 / 10 / 04$ & Thin & Small window & 38.3 & $\times$ \\
\hline 1513 & 0412600401 & $2008 / 03 / 13$ & Thin & Small window & 63.5 & \\
\hline 1616 & 0412600601 & $2008 / 10 / 04$ & Thin & Small window & 62.5 & \\
\hline
\end{tabular}




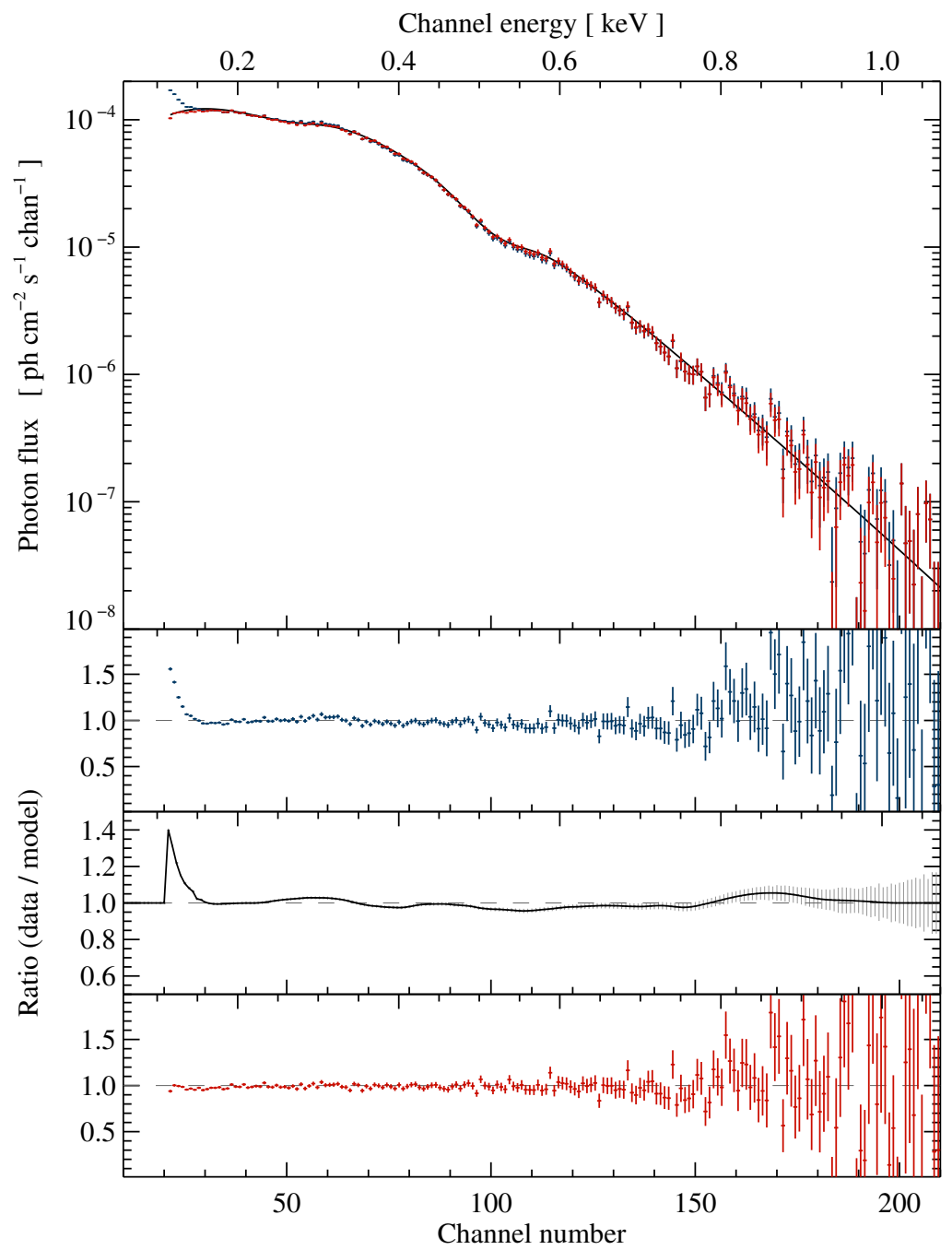

Figure B.1: EPIC/pn spectrum of RXJ1856.4-3754 on September 24, 2005 (rev. 1061) in small window mode and thin filter. 
Table B.2: Archival XMM-Newton EPIC/MOS1 observations of the white dwarf HZ $43 \mathrm{~A}$ and the isolated neutron star RX J1856.4-3754.

\begin{tabular}{|c|c|c|c|c|c|c|}
\hline Cycle & Obs. ID & Date & Filter & Mode & \multicolumn{2}{|c|}{$t_{\text {exp }}[\mathrm{ksec}]$} \\
\hline \multicolumn{7}{|c|}{$H Z 43$ A } \\
\hline \multirow[t]{4}{*}{0089} & 0125970101 & 2000/06/04 & Thin & Timing & 5.0 & \\
\hline & & & Medium & Timing & 5.7 & \\
\hline & & & Thick & Timing & 1.9 & \\
\hline & & & Thick & Timing & 1.7 & \\
\hline 0928 & 0212090101 & 2005/01/03 & Thin & Small window & 1.7 & \\
\hline \multicolumn{7}{|c|}{ RXJ1856 } \\
\hline 0427 & 0106260101 & 2002/04/08 & Thin & Timing & 57.7 & \\
\hline 0798 & 0201590101 & $2004 / 04 / 17$ & Thin & Full frame & 66.3 & \\
\hline \multirow[t]{2}{*}{0878} & 0165971601 & $2004 / 09 / 24$ & Thin & Small window & 33.2 & \\
\hline & 0165971701 & 2004/09/24 & Medium & Small window & 36.7 & \\
\hline 0968 & 0165971901 & $2005 / 03 / 23$ & Thin & Large window & 35.2 & \\
\hline 0980 & 0213080101 & 2005/04/15 & Thin & Large window & 8.8 & \\
\hline 1061 & 0165972001 & $2005 / 09 / 24$ & Thin & Small window & 33.3 & $x$ \\
\hline 1153 & 0165972101 & $2006 / 03 / 26$ & Thin & Small window & 69.8 & $\times$ \\
\hline 1259 & 0412600101 & $2006 / 10 / 24$ & Thin & Small window & 72.8 & $x$ \\
\hline 1330 & 0412600201 & 2007/03/14 & Thin & Small window & 68.2 & $x$ \\
\hline 1335 & 0415180101 & $2007 / 03 / 25$ & Thin & Large window & 40.7 & \\
\hline 1432 & 0412600301 & 2007/10/04 & Thin & Small window & 39.0 & $x$ \\
\hline 1513 & 0412600401 & $2008 / 03 / 13$ & Thin & Small window & 62.7 & \\
\hline 1616 & 0412600601 & 2008/10/04 & Thin & Small window & 63.0 & \\
\hline
\end{tabular}




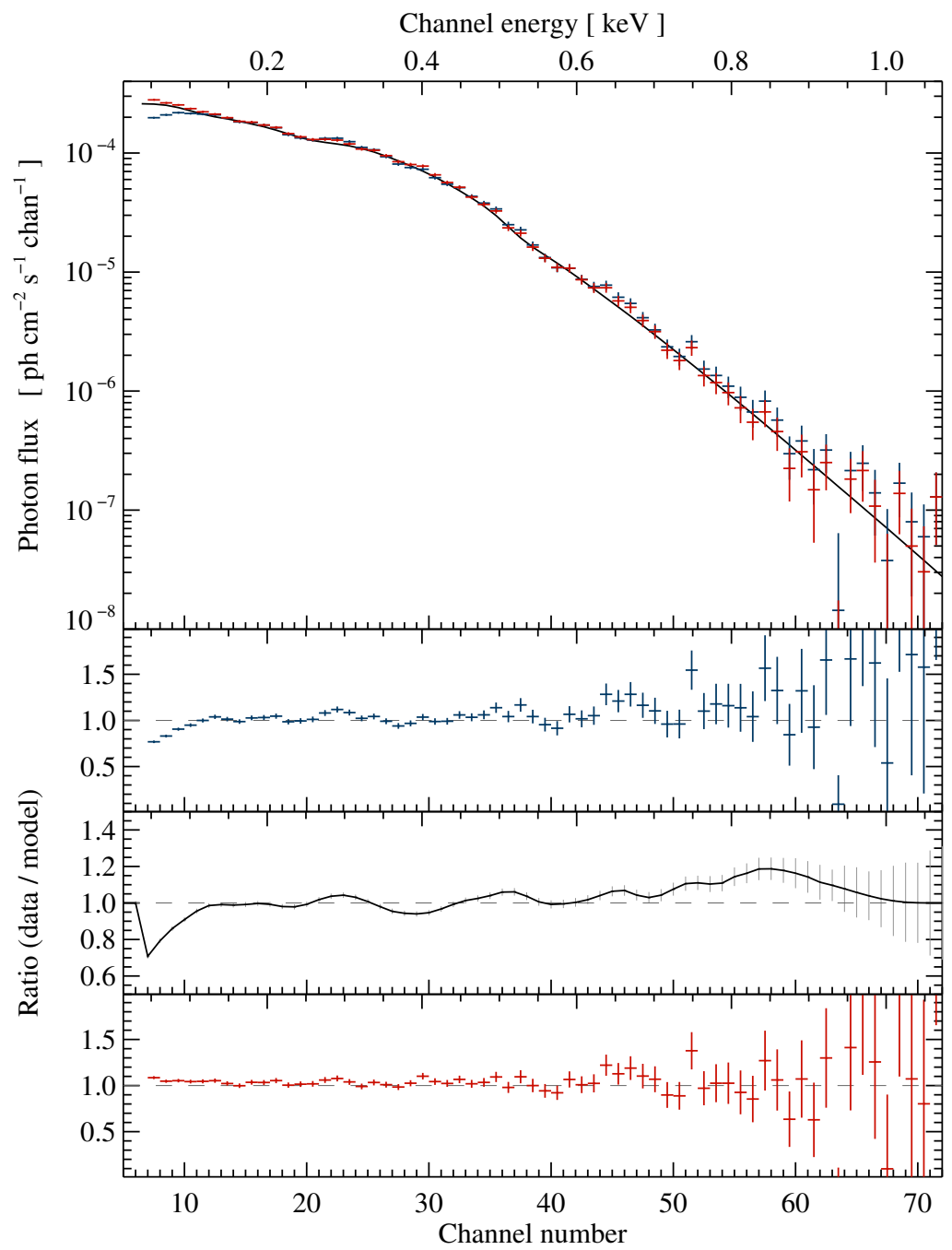

Figure B.2: EPIC/MOS1 spectrum of RXJ1856.4-3754 on September 24, 2005 (rev. 1061) in small window mode and thin filter. 
Table B.3: Archival XMM-Newton EPIC/MOS2 observations of the white dwarf HZ $43 \mathrm{~A}$ and the isolated neutron star RX J1856.4-3754.

\begin{tabular}{|c|c|c|c|c|c|c|}
\hline Cycle & Obs. ID & Date & Filter & Mode & \multicolumn{2}{|c|}{$t_{\exp }[\mathrm{ksec}]$} \\
\hline \multicolumn{7}{|c|}{ HZ43 A } \\
\hline \multirow[t]{4}{*}{0089} & 0125970101 & 2000/06/04 & Thin & Timing & 5.0 & \\
\hline & & & Medium & Timing & 5.7 & \\
\hline & & & Thick & Timing & 1.9 & \\
\hline & & & Thick & Timing & 0.9 & \\
\hline 0928 & 0212090101 & 2005/01/03 & Thin & Small window & 1.8 & \\
\hline \multicolumn{7}{|c|}{$R X J 1856$} \\
\hline 0427 & 0106260101 & 2002/04/08 & Thin & Small window & 58.0 & \\
\hline 0798 & 0201590101 & 2004/04/17 & Thin & Full frame & 66.4 & \\
\hline \multirow[t]{2}{*}{0878} & 0165971601 & $2004 / 09 / 24$ & Thin & Small window & 33.2 & \\
\hline & 0165971701 & $2004 / 09 / 24$ & Medium & Small window & 36.7 & \\
\hline 0968 & 0165971901 & $2005 / 03 / 23$ & Thin & Small window & 35.2 & \\
\hline 0980 & 0213080101 & 2005/04/15 & Thin & Large window & 7.7 & \\
\hline 1061 & 0165972001 & $2005 / 09 / 24$ & Thin & Small window & 33.3 & $x$ \\
\hline 1153 & 0165972101 & $2006 / 03 / 26$ & Thin & Small window & 69.8 & $x$ \\
\hline 1259 & 0412600101 & $2006 / 10 / 24$ & Thin & Small window & 72.8 & $x$ \\
\hline 1330 & 0412600201 & 2007/03/14 & Thin & Small window & 68.2 & $x$ \\
\hline 1335 & 0415180101 & $2007 / 03 / 25$ & Thin & Large window & 40.7 & \\
\hline 1432 & 0412600301 & 2007/10/04 & Thin & Small window & 38.5 & $x$ \\
\hline 1513 & 0412600401 & 2008/03/13 & Thin & Small window & 62.7 & \\
\hline 1616 & 0412600601 & 2008/10/04 & Thin & Small window & 63.0 & \\
\hline
\end{tabular}




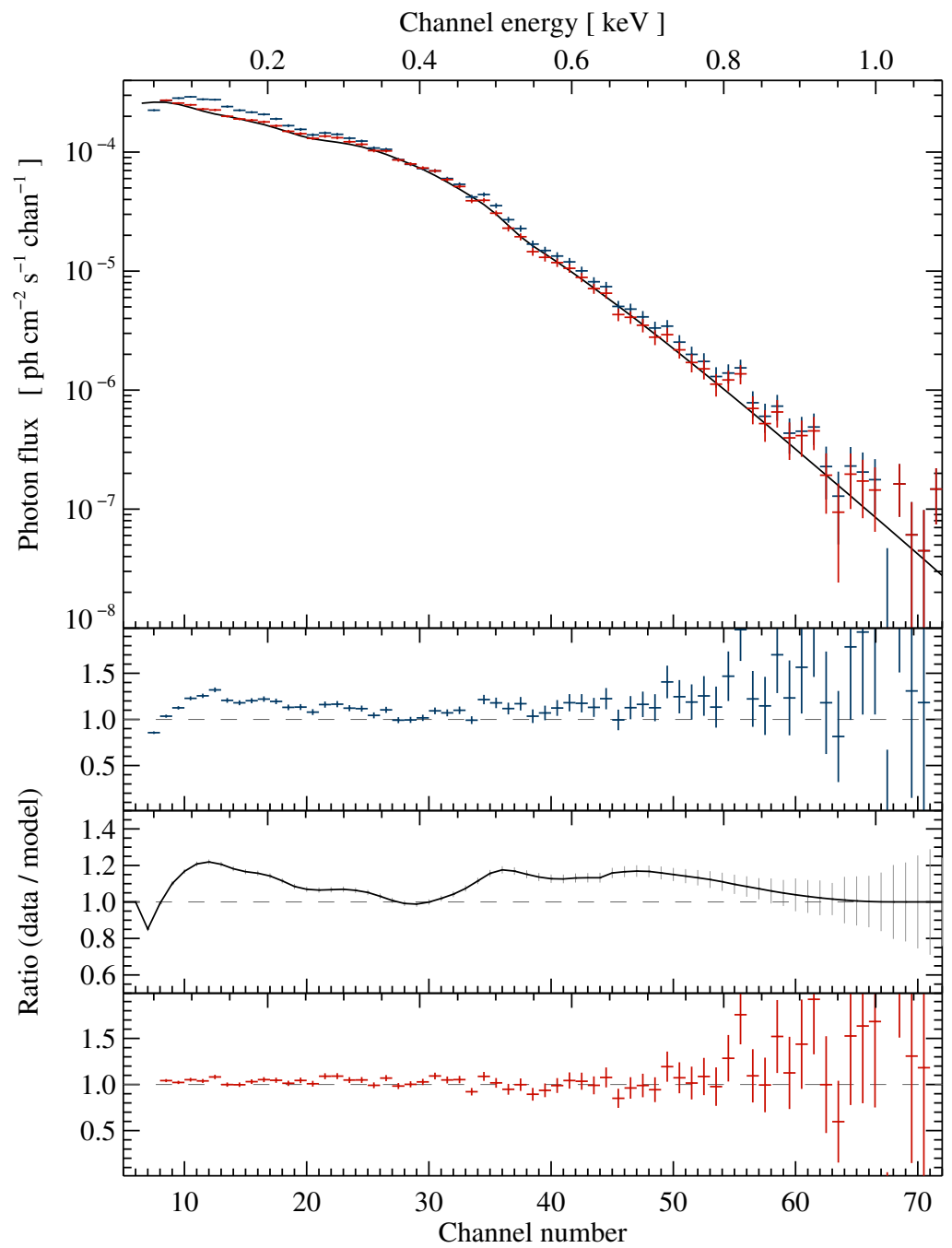

Figure B.3: EPIC/MOS2 spectrum of RXJ1856.4-3754 on September 24, 2005 (rev. 1061) in small window mode and thin filter. 


\section{BIBLIOGRAPHY}

Aizu, K. 1973, Progr. Theor. Phys., 49, 1184

Ak, T., Bilir, S., Ak, S., Retter, A. 2007, New Astronomy, 12, 446

Anders, E., Grevesse, N. 1989, Geochim. Cosmochim. Acta, 53, 197

Arnaud, K., Dorman, B., Gordon, C. 2008, XSPEC, An X-Ray Spectral Fitting Package. User's Guide for version 12.5.0, HEASARC, Astrophysics Science Division, NASA/GSFC

Arnaud, K. A. 1996, in Astronomical Data Analysis Software and Systems V, eds. G. H. Jacoby and J. Barnes, ASP Conf. Ser., 101, 17

Arnaud, M., Raymond, J. 1992, ApJ, 398, 394

Arnaud, M., Rothenflug, R. 1985, A\&AS, 60, 425

Balucinska-Church, M., McCammon, D. 1992, ApJ, 400, 699

Barstow, M. A., Holberg, J. B., Koester, D. 1995, MNRAS, 274, L31

Berg, R. A., Duthie, J. G. 1977, ApJ, 211, 859

Beuermann, K. 1999, in Highlights in X-ray Astronomy, eds. B. Aschenbach and M. J. Freyberg, MPE Report, 272, 410

Beuermann, K. 2004, in IAU Colloq. 190: Magnetic Cataclysmic Variables, eds. S. Vrielmann and M. Cropper, ASP Conf. Ser., 315, 187

Beuermann, K. 2008, A\&A, 481, 919

Beuermann, K., Burwitz, V. 1995, in Cape Workshop on Magnetic Cataclysmic Variables, eds. D. A. H. Buckley and B. Warner, ASP Conf. Ser., 85, 99

Beuermann, K., Burwitz, V., Rauch, T. 2006, A\&A, 458, 541

Beuermann, K., Burwitz, V., Rauch, T. 2008, A\&A, 481, 769

Beuermann, K., Schwope, A. D. 1994, in Interacting Binary Stars, ed. A. W. Shafter, ASP Conf. Ser., 56, 119

Beuermann, K., Thomas, H.-C. 1993, Adv. Space Res., 13, 115 
Bevington, P. R., Robinson, D. K. 2003, Data reduction and error analysis for the physical sciences (McGraw-Hill Education)

Briel, U. G., Aschenbach, B., Balasini, M., et al. 2000, in X-Ray Optics, Instruments, and Missions III, eds. J. E. Truemper and B. Aschenbach, SPIE, 4012, 154

Buckley, D. A. H., O’Donoghue, D., Hassall, B. J. M., et al. 1993, MNRAS, 262, 93

Burwitz, V., Haberl, F., Neuhäuser, R., et al. 2003, A\&A, 399, 1109

Campbell, C. G. 1985, MNRAS, 215, 509

Campbell, C. G., Schwope, A. D. 1999, A\&A, 343, 132

Chanmugam, G., Langer, S. H., Shaviv, G. 1985, ApJ, 299, L87

Chanmugam, G., Ray, A. 1984, ApJ, 285, 252

Chanmugam, G., Wagner, R. L. 1977, ApJ, 213, L13

Charles, P. A., Seward, F. D. 1995, Exploring the X-ray universe (Cambridge University Press, Cambridge New York)

Cropper, M. 1988, MNRAS, 231, 597

Cropper, M. 1990, Space Sci. Rev., 54, 195

Cropper, M., Ramsay, G., Hellier, C., et al. 2002, Phil. Trans. R. Soc. Lond. A, 360, 1951

Cropper, M., Wu, K., Ramsay, G., Kocabiyik, A. 1999, MNRAS, 306, 684

Dickey, J. M., Lockman, F. J. 1990, ARA\&A, 28, 215

Done, C., Mulchaey, J. S., Mushotzky, R. F., Arnaud, K. A. 1992, ApJ, 395, 275

Done, C., Osborne, J. P., Beardmore, A. P. 1995, MNRAS, 276, 483

Dorman, B., Arnaud, K. A. 2001, in Astronomical Data Analysis Software and Systems X, eds. F. R. Harnden, Jr., F. A. Primini, and H. E. Payne, ASP Conf. Ser., 238, 415

Dorman, B., Arnaud, K. A., Gordon, C. A. 2003, in Bull. Am. Astron. Soc., 35, 641

Dupuis, J., Vennes, S., Chayer, P., Hurwitz, M., Bowyer, S. 1998, ApJ, 500, L45

Eggleton, P. P. 1983, ApJ, 268, 368

Ehle, M., Breitfellner, M., Díaz Trigo, M., et al. 2007, XMM-Newton Users' Handbook, Issue 2.5, ESA, Doc. No. XMM-PS-GM-14

Euchner, F., Jordan, S., Beuermann, K., Gänsicke, B. T., Hessman, F. V. 2002, A\&A, 390,633

Ferrario, L., Wickramasinghe, D. T., Bailey, J. A., Buckley, D. A. H. 1994, MNRAS, 268,128

Fischer, A., Beuermann, K. 2001, A\&A, 373, 211 
Frank, J., King, A., Raine, D. J. 2002, Accretion Power in Astrophysics: Third Edition (Cambridge University Press, Cambridge (UK))

Frank, J., King, A. R., Lasota, J.-P. 1988, A\&A, 193, 113

Friedman, H., Lichtman, S. W., Byram, E. T. 1951, Phys. Rev., 83, 1025

Friedrich, P. 2008, in The Universe in X-Rays, eds. J. E. Trümper and G. Hasinger (Springer, Berlin Heidelberg New York), 41

Gänsicke, B. T. 1998, in Wild Stars in the Old West, eds. S. Howell, E. Kuulkers, and C. Woodward, ASP Conf. Ser., 137, 88

García, J., Mendoza, C., Bautista, M. A., et al. 2005, ApJS, 158, 68

Gerke, J. R., Howell, S. B., Walter, F. M. 2006, PASP, 118, 678

Giacconi, R. 2003, Rev. Mod. Phys., 75, 995

Giacconi, R., Gursky, H., Paolini, F. R., Rossi, B. B. 1962, Phys. Rev. Lett., 9, 439

Grevesse, N., Asplund, M., Sauval, A. J. 2007, Space Sci. Rev., 130, 105

Haberl, F. 2007, Ap\&SS, 308, 181

Hameury, J. M., King, A. R., Lasota, J. P., Ritter, H. 1988, MNRAS, 231, 535

Hearn, D. R., Richardson, J. A., Bradt, H. V. D., et al. 1976, ApJ, 203, L21

Hearn, D. R., Richardson, J. A., Condon, J., Liebert, J., Spinrad, H. 1975, IAU Circ., 2790, 1

den Herder, J. W., Brinkman, A. C., Kahn, S. M., et al. 2001, A\&A, 365, L7

Hessman, F. V., Gänsicke, B. T., Mattei, J. A. 2000, A\&A, 361, 952

Humason, M. L., Zwicky, F. 1947, ApJ, 105, 85

Jansen, F., Lumb, D., Altieri, B., et al. 2001, A\&A, 365, L1

Joy, A. H. 1954, ApJ, 120, 377

Joy, A. H. 1956, ApJ, 124, 317

Kafka, S., Honeycutt, R. K. 2005, AJ, 130, 742

Kalberla, P. M. W., Burton, W. B., Hartmann, D., et al. 2005, A\&A, 440, 775

Kallman, T., Bautista, M. 2001, ApJS, 133, 221

Karzas, W. J., Latter, R. 1961, ApJS, 6, 167

Katajainen, S., Scaltriti, F., Piirola, V., Lehto, H. J., Anderlucci, E. 2001, A\&A, 372, 945

Kellogg, E., Baldwin, J. R., Koch, D. 1975, ApJ, 199, 299

van Kerkwijk, M. H., Kulkarni, S. R. 2001, A\&A, 380, 221 
King, A. R. 1988, Q. J. R. Astron. Soc., 29, 1

King, A. R., Lasota, J. P. 1979, MNRAS, 188, 653

King, A. R., Lasota, J. P. 1984, A\&A, 140, L16

Kippenhahn, R., Weigert, A. 1990, Stellar Structure and Evolution (Springer, Berlin Heidelberg New York)

Kirsch, M. G. F. 2007, EPIC status of calibration and data analysis, Issue 2.6, ESA, Doc. No. XMM-SOC-CAL-TN-0018

Knigge, C. 2006, MNRAS, 373, 484

de Kool, M. 1992, A\&A, 261, 188

Kopal, Z. 1955, Annales d'Astrophysique, 18, 379

Kraft, R. P. 1962, ApJ, 135, 408

Kraft, R. P., Mathews, J., Greenstein, J. L. 1962, ApJ, 136, 312

Krzeminski, W., Serkowski, K. 1977, ApJ, 216, L45

Kuijpers, J., Pringle, J. E. 1982, A\&A, 114, L4

Kuntz, K. D., Snowden, S. L. 2008, A\&A, 478, 575

Lamb, D. Q., Masters, A. R. 1979, ApJ, 234, L117

Lampton, M., Margon, B., Paresce, F., Stern, R., Bowyer, S. 1976, ApJ, 203, L71

Langer, S. H., Chanmugam, G., Shaviv, G. 1981, ApJ, 245, L23

Levenberg, K. 1944, Quart. Appl. Math., 2, 164

Liebert, J., Stockman, H. S. 1985, in Cataclysmic Variables and Low-Mass X-ray Binaries, eds. D. Q. Lamb and J. Patterson (Reidel, Dordrecht), 151

Liedahl, D. A., Osterheld, A. L., Goldstein, W. H. 1995, ApJ, 438, L115

Livio, M., Pringle, J. E. 1994, ApJ, 427, 956

Loiseau, N., Ehle, M., Pollock, A., et al. 2007, User's Guide to the XMM-Newton Science Analysis System, Issue 4.1, ESA, Doc. No. SAS USG

Lomb, N. R. 1976, Ap\&SS, 39, 447

de Luca, A., Molendi, S. 2004, A\&A, 419, 837

Lumb, D. H., Warwick, R. S., Page, M., de Luca, A. 2002, A\&A, 389, 93

Margon, B., Liebert, J., Lampton, M., et al. 1976, ApJ, 209, 525

Marquardt, D. 1963, J. Soc. Indust. Appl. Math., 11, 431

de Martino, D., Mouchet, M., Rosen, S. R., et al. 1998, A\&A, 329, 571 
Mason, K. O., Breeveld, A., Much, R., et al. 2001, A\&A, 365, L36

Mewe, R., Gronenschild, E. H. B. M., van den Oord, G. H. J. 1985, A\&AS, 62, 197

Mewe, R., Lemen, J. R., van den Oord, G. H. J. 1986, A\&AS, 65, 511

Motl, P. 2001, Numerical Simulations of Dynamical Mass Transfer in Binaries, Dissertation, Louisiana State University

Mushotzky, R. F., Szymkowiak, A. E. 1988, in Cooling Flows in Clusters and Galaxies, ed. A. C. Fabian, NATO ASIC Proc., 229, 53

Napiwotzki, R., Barstow, M. A., Fleming, T., et al. 1993, A\&A, 278, 478

O’Dwyer, I. J., Chu, Y.-H., Gruendl, R. A., Guerrero, M. A., Webbink, R. F. 2003, AJ, 125,2239

Osborne, J. P., Beuermann, K., Charles, P., et al. 1987, ApJ, 315, L123

Paczyński, B. 1976, in Structure and Evolution of Close Binary Systems, eds. P. Eggleton, S. Mitton, and J. Whelan, IAU Symp., 73, 75

Ramsay, G., Cropper, M. 2002, MNRAS, 334, 805

Ramsay, G., Cropper, M. 2003, MNRAS, 338, 219

Ramsay, G., Cropper, M. 2004, MNRAS, 347, 497

Ramsay, G., Cropper, M. 2007, MNRAS, 379, 1209

Ramsay, G., Cropper, M., Mason, K. O., Córdova, F. A., Priedhorsky, W. 2004, MNRAS, 347, 95

Ramsay, G., Mason, K. O., Cropper, M., Watson, M. G., Clayton, K. L. 1994, MNRAS, 270, 692

Rappaport, S., Verbunt, F., Joss, P. C. 1983, ApJ, 275, 713

Read, A. M., Ponman, T. J. 2003, A\&A, 409, 395

Read, A. M., Sembay, S. F., Abbey, T. F., Turner, M. J. L. 2006, in The X-ray Universe 2005, ed. A. Wilson, ESA Special Publication, 604, 925

Reilman, R. F., Manson, S. T. 1979, ApJS, 40, 815

Ritter, H., Kolb, U. 2003, A\&A, 404, 301

Romero-Colmenero, E., Potter, S. B., Buckley, D. A. H. 2005, in The Astrophysics of Cataclysmic Variables and Related Objects, eds. J.-M. Hameury and J.-P. Lasota, ASP Conf. Ser., 330, 429

Rosen, S. R., Mittaz, J. P. D., Buckley, D. A., et al. 1996, MNRAS, 280, 1121

Rosen, S. R., Rainger, J. F., Burleigh, M. R., et al. 2001, MNRAS, 322, 631

Rothschild, R. E., Gruber, D. E., Knight, F. K., et al. 1981, ApJ, 250, 723 
Rousseau, T., Fischer, A., Beuermann, K., Woelk, U. 1996, A\&A, 310, 526

Scargle, J. D. 1982, ApJ, 263, 835

Schuh, S. L., Dreizler, S., Deetjen, J. L., Göhler, E. 2003, Balt. Astron., 12, 167

Schwarz, R. 1996, Spektroskopische und photometrische Beobachtungen des AM Herculis-Sterns RX J0203.8+2959, Diploma thesis, Humboldt-Universität Berlin

Schwarz, R., Reinsch, K., Beuermann, K., Burwitz, V. 2005, A\&A, 442, 271

Schwarz, R., Schwope, A. D., Beuermann, K., et al. 1998, A\&A, 338, 465

Schwarz, R., Schwope, A. D., Staude, A. 2001, A\&A, 374, 189

Schwarz, R., Schwope, A. D., Staude, A., et al. 2007, A\&A, 473, 511

Schwope, A. D., Schwarz, R., Sirk, M., Howell, S. B. 2001, A\&A, 375, 419

Schwope, A. D., Thomas, H.-C., Beuermann, K., et al. 1995, A\&A, 293, 764

Shull, J. M., van Steenberg, M. 1982, ApJS, 48, 95

Smith, M. J. S. 2004, PN X-Ray Loading, Issue 1.0, ESA, Doc. No. XMM-SOC-CAL$\mathrm{TN}-0050$

Smith, M. J. S. 2008, PN Optical Loading, Issue 1.2, ESA, Doc. No. XMM-SOC-CALTN-0051

Snowden, S., Shafer, R., Smith, R., et al. 2008, The XMM-Newton ABC Guide: An Introduction to XMM-Newton Data Analysis, Version 4.1 for XMM-SAS v8.0, NASA/GSFC XMM-Newton Guest Observer Facility

Spruit, H. C., Ritter, H. 1983, A\&A, 124, 267

Standish, E. M. 1998, JPL IOM, 312.F-98-048

Strüder, L., Briel, U., Dennerl, K., et al. 2001, A\&A, 365, L18

Strüder, L., Meidinger, N., Pfeffermann, E., et al. 2000, in X-Ray Optics, Instruments, and Missions III, eds. J. E. Truemper and B. Aschenbach, SPIE, 4012, 342

Stuhlinger, M., Kirsch, M. G. F., Santos-Lleo, M., et al. 2008, Status of XMM-Newton instrument cross-calibration with SAS v7.1, Issue 5.0, ESA, Doc. No. XMM-SOCCAL-TN-0052

Taam, R. E., Spruit, H. C. 1989, ApJ, 345, 972

Tapia, S. 1977, ApJ, 212, L125

van Teeseling, A., Heise, J., Paerels, F. 1994, A\&A, 281, 119

Tiengo, A., Mereghetti, S. 2007, ApJ, 657, L101

Tout, C. A., Wickramasinghe, D. T., Liebert, J., Ferrario, L., Pringle, J. E. 2008, MNRAS, 387, 897 
Traulsen, I., Reinsch, K., Dreizler, S., Schwarz, R. 2007, in 15th European Workshop on White Dwarfs, eds. R. Napiwotzki and M. R. Burleigh, ASP Conf. Ser., 372, 553

Trümper, J. 1990, Physics Bulletin, 46, 137

Turner, M. J. L., Abbey, A., Arnaud, M., et al. 2001, A\&A, 365, L27

Verbunt, F., Zwaan, C. 1981, A\&A, 100, L7

Verner, D. A., Ferland, G. J. 1996, ApJS, 103, 467

Verner, D. A., Ferland, G. J., Korista, K. T., Yakovlev, D. G. 1996, ApJ, 465, 487

Vogel, J., Byckling, K., Schwope, A., et al. 2008, A\&A, 485, 787

Voges, W., Aschenbach, B., Boller, T., et al. 1999, A\&A, 349, 389

Walker, M. F. 1954, PASP, 66, 230

Walter, F. M., Wolk, S. J., Neuhäuser, R. 1996, Nature, 379, 233

Warner, B. 1972, MNRAS, 158, 425

Warner, B. 2003, Cataclysmic Variable Stars (Cambridge University Press, Cambridge (UK))

Warren, J. K., Sirk, M. M., Vallerga, J. V. 1995, ApJ, 445, 909

Warren, J. K., Vallerga, J. V., Mauche, C. W., Mukai, K., Siegmund, O. H. W. 1993, ApJ, 414, L69

Webbink, R. F., Wickramasinghe, D. T. 2002, MNRAS, 335, 1

Werner, K., Deetjen, J. L., Dreizler, S., et al. 2003, in Stellar Atmosphere Modeling, eds. I. Hubeny, D. Mihalas, and K. Werner, ASP Conf. Ser., 288, 31

White, N. E., Giommi, P., Angelini, L. 2000, VizieR Online Data Catalog, 9031

Wickramasinghe, D. T., Ferrario, L. 2005, MNRAS, 356, 1576

Wilms, J., Allen, A., McCray, R. 2000, ApJ, 542, 914

Woelk, U., Beuermann, K. 1992, A\&A, 256, 498

Woelk, U., Beuermann, K. 1996, A\&A, 306, 232

Wu, K., Chanmugam, G., Shaviv, G. 1994, ApJ, 426, 664

Yan, M., Sadeghpour, H. R., Dalgarno, A. 1998, ApJ, 496, 1044

Zdziarski, A. A., Johnson, W. N., Done, C., Smith, D., McNaron-Brown, K. 1995, ApJ, 438, L63

Zimmermann, H. U., Belloni, T., Izzo, C., Kahabka, P., Schwentker, O. 1993, in Astronomical Data Analysis Software and Systems II, eds. R. J. Hanisch, R. J. V. Brissenden, and J. Barnes, ASP Conf. Ser., 52, 233 


\section{ACKNOWLEDGMENTS}

Many persons made my $\mathrm{PhD}$ years a precious time and contributed to the course of this work. To all of them, I want to express my gratitude.

Stefan Dreizler has been my supervisor and teacher for years and offered me the opportunity to join his group in Göttingen. His continued interest and his always being available for aid benefit my work. Klaus Reinsch initiated the project and has guided and encouraged me in many ways. I much appreciate their motivating advice and support.

Klaus Beuermann enriched the work with his suggestions, comments, and explanations. Our collaborators Robert Schwarz, Axel Schwope, and Vadim Burwitz have provided useful remarks and discussions. Fred Walter, Frederic H. Hessman, and Thorsten Nagel carried out the optical observations at the SMARTS, MONET/North, and Tübingen telescopes. Since the starting days in Tübingen, Sonja Schuh supplies me with her helpfulness and her mentoring.

I am deeply thankful to my family and friends for their backing, encouragement, and patience; in particular to my parents Hans-Dietrich and Monika Traulsen, to Sylvia Brandert, Jürgen and Susanne Büchsel, Sonja Fritz, Agnes Hoffmann, Alexandra and Imke Kaasch, Katharina Lesch, Eva and Hans Scholz, Matthias, Christian and Susanne Traulsen.

This research has been granted by DLR under project number 50 OR 0501 and is based on observations obtained with XMM-Newton, an ESA science mission with instruments and contributions directly funded by ESA Member States and NASA. 


\section{Curriculum Vitae}

\begin{tabular}{|c|c|}
\hline Geburtsdatum & 3. Mai 1978 \\
\hline Geburtsort & Stuttgart \\
\hline Staatsangehörigkeit & deutsch \\
\hline $09 / 1984-08 / 1988$ & Grundschule Im Sonnigen Winkel, Stuttgart \\
\hline $09 / 1988-06 / 1997$ & Karls-Gymnasium Stuttgart \\
\hline $06 / 1997$ & Allgemeine Hochschulreife \\
\hline $10 / 1997-07 / 1998$ & Studium generale am Leibniz-Kolleg Tübingen \\
\hline $10 / 1998-07 / 2004$ & $\begin{array}{l}\text { Physikstudium (Diplom) an der Eberhard-Karls- } \\
\text { Universität Tübingen }\end{array}$ \\
\hline $03 / 2001$ & Vordiplom (Wahlfach Informatik) \\
\hline $10 / 2000-09 / 2005$ & $\begin{array}{l}\text { Institut für Astronomie und Astrophysik Tübingen, } \\
\text { Abteilung Astronomie }\end{array}$ \\
\hline $10 / 2000-06 / 2003$ & Studentische Hilfskraft \\
\hline $07 / 2003-07 / 2004$ & $\begin{array}{l}\text { Diplomarbeit "Metallhäufigkeiten in heißen wasser- } \\
\text { stoffreichen Zentralsternen Planetarischer Nebel" }\end{array}$ \\
\hline $07 / 2004$ & $\begin{array}{l}\text { Diplom-Physikerin (Schwerpunktfach Astronomie } \\
\text { Astrophysik, Wahlfach Mathematik) }\end{array}$ \\
\hline $01 / 2005-02 / 2005$ & $\begin{array}{l}\text { Wissenschaftliche Mitarbeiterin am Max-Planck- } \\
\text { Institut für Sonnensystemforschung Katlenburg- } \\
\text { Lindau }\end{array}$ \\
\hline $06 / 2005$ & $\begin{array}{l}\text { Wissenschaftliche Hilfskraft am Institut für Astro- } \\
\text { nomie und Astrophysik Tübingen }\end{array}$ \\
\hline $10 / 2005-03 / 2009$ & $\begin{array}{l}\text { Promotionsstudium an der Georg-August-Universi- } \\
\text { tät Göttingen, Fakultät für Physik }\end{array}$ \\
\hline $10 / 2005-$ & $\begin{array}{l}\text { Wissenschaftliche Mitarbeiterin am Institut für } \\
\text { Astrophysik Göttingen }\end{array}$ \\
\hline
\end{tabular}


132

Curriculum vitae 